\title{
A Method to Characterize Skeletal Muscle Contractile Properties Using a Wearable Ultrasonic Sensor
}

\author{
by \\ Elliot Yeung \\ A thesis submitted to the \\ Faculty of Graduate Studies and Research \\ in partial fulfillment of the requirements for the degree of \\ Master of Applied Science in Biomedical Engineering \\ Ottawa-Carleton Institute of Biomedical Engineering (OCIBME) \\ Department of Systems and Computer Engineering \\ Carleton University \\ Ottawa, Ontario, Canada
}

(C)2020, Elliot Yeung 


\section{Abstract}

Skeletal muscle monitoring provides valuable information about the muscle contractile properties which could be useful in various clinical applications. A wearable ultrasonic sensor (WUS) was constructed using a $110 \mu \mathrm{m}$ thick polyvinylidene fluoride polymer piezoelectric film to perform a continuous hands-free muscle monitoring. The lightweight and flexible properties of WUS enables the stable attachment to the skin surface without affecting the tissue motion of interest, which results in reduced motion artifacts. In order to perform reliable estimation of tissue thicknesses by the WUS in an ultrasonic pulse echo mode, the accuracy of selected signal analysis techniques was evaluated using a laser displacement measurement result as a reference. Then, a mechanical simulation experiment is conducted to evaluate the accuracy and repeatability of the WUS measurement system. In addition, the effects of moving averaging on the signal-to-noise ratio of the ultrasonic signals and the estimation accuracy of the tissue thickness were investigated. In order to demonstrate the feasibility of the proposed ultrasound technique for in-vivo measurements, continuous monitoring of the lateral head of triceps muscle of three healthy male subjects were monitored using the WUS. The tissue thickness changes due to the evoked muscle contraction by an electromyostimulation (EMS) at various pulse repetition frequencies were monitored. 


\section{Acknowledgements}

I would like to express my deep and sincere gratitude to the people who have helped me in the completion of the thesis.

Firstly, I would begin by thanking my supervisor Prof. Yuu Ono for his guidance throughout the course of my M.A.Sc. Prof. Ono who has patiently spent countless hours discussing problems and guiding me to understand research methods and problem solving.

I would like to extend my gratitude towards Mr. Ibrahim Almohimeed, who has been extremely supportive in providing research materials and exchanging ideas. Mr. Almohimeed has taken personal efforts in my research by being a co-author of the publications with me.

I thank Mr. Alex Proctor for assisting and technical supporting me in designing the mechanical phantom.

I would like to thank my friends, Mr. Dipayan Mitra, Mr. Sean Trudel, and Mr. Shanmugaraja K.V who helped me in all possible ways. I would also like to thank Carleton University for supporting the work. I would like to express my gratitude towards my parents and my brother for their unwavering support. 


\section{Contents}

Contents iv

List of Figures viii

List of Tables $\quad$ Xv

1 Introduction 1

1.1 Overview ............................... 1

1.2 Problem Statement . . . . . . . . . . . . . . . . . . . 2

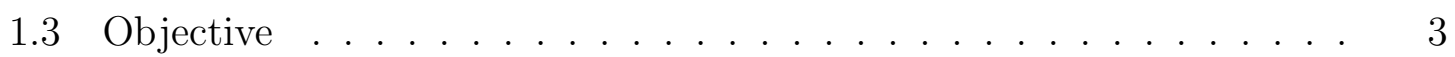

1.4 Thesis Contributions .................... 4

1.5 Research Publications . . . . . . . . . . . . . . . 5

1.6 Thesis Organization . . . . . . . . . . . . . . 5

2 Background Review $\quad 6$

2.1 Human Muscle Physiology . . . . . . . . . . . . . . . 6

2.2 Skeletal Muscle Contraction and Relaxation . . . . . . . . . . . 7

2.2.1 Electrical Stimulus in Muscle Contraction . . . . . . . . . . 9

2.2.2 Single Muscle Twitch and Fused Muscle Contraction . . . . . 10

2.2.3 Muscle Fiber Types . . . . . . . . . . . . . . . . . . . . . . 12 
2.2 .4 Fusion Index . . . . . . . . . . . . . . . . . . . 13

2.3 Existing Methods of Monitoring Skeletal Muscle Behavior . . . . . . 16

2.3.1 Surface Electromyogram $(\mathrm{sEMG}) \ldots \ldots$. . . . . . . . 17

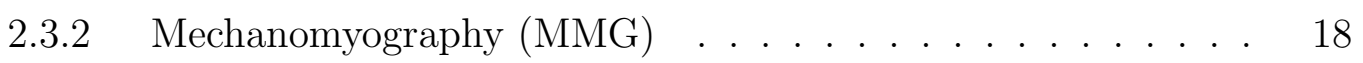

2.3.3 Magnetic Resonance Imaging (MRI) . . . . . . . . . . . . . . 19

2.3.4 Ultrasonography .................... 19

2.4 Ultrasound Basic . . . . . . . . . . . . . . . . . . . . 20

2.4.1 Ultrasound Attenuation ............... 23

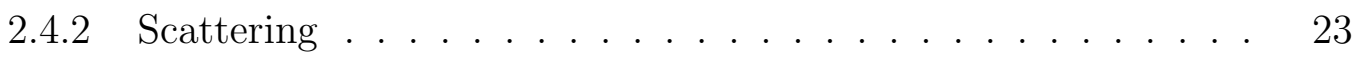

2.4.3 Hand-Held Conventional Ultrasound Probe . . . . . . . . . . . 24

3 Wearable Ultrasonic Sensor and DSP Techniques 26

3.1 Wearable Ultrasound Sensor . . . . . . . . . . . . . . . . 26

3.2 Pre-processing Technique . . . . . . . . . . . . . . . 32

3.3 Thickness Changes Estimation Methods . . . . . . . . . . 33

3.3.1 Peak Detection .................... 34

3.3.2 Cross Correlation .................. 34

3.3.3 Quadrature Demodulation .............. 36

4 Accuracy Evaluation on Thickness Measurement Using Gelatin$\begin{array}{ll}\text { based Phantom } & 39\end{array}$

4.1 Soft Tissue Mimicking Phantom . . . . . . . . . . . . . . . . . 40

4.1.1 Review of Gelatin-Based Phantom . . . . . . . . . . . . . . . 40

4.1 .2 Phantom Construction . . . . . . . . . . . . . . 42

4.1.3 Phantom Ultrasound Velocity and Acoustic Attenuation . . . 44

4.2 Selection of the DSP Techniques for Thickness Measurement Based on Accuracy Evaluation . . . . . . . . . . . . . . . . . . 49 
4.2.1 Experimental Configuration for Single Twitch Phantom Simu-

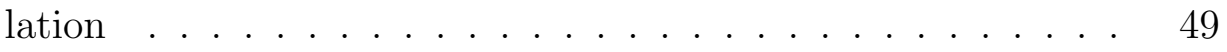

4.2.2 Comparison of the DSP Techniques . . . . . . . . . . 52

4.3 Accuracy Evaluation Using Phantom Experimental Environment . . . 54

4.3.1 Experimental Setup for Thickness Measurement Accuracy Evaluation Using a Phantom . . . . . . . . . . . . . 55

4.3.2 Repeatability and Accuracy Evaluation for Dynamic Contraction 62

\section{In-vivo Experiments for Monitoring Skeletal Muscle Contraction}

$\begin{array}{ll}\text { Using a Wearable Ultrasound Sensor } & 70\end{array}$

5.1 Effect of Moving Averaging on Thickness Measurement Accuracy . . 71

5.2 Review of Electromyostimulation . . . . . . . . . . . . 77

5.3 Experimental Configuration in Muscle Monitoring with Electromyostimulation ......................... 80

5.4 Experimental Result . . . . . . . . . . . . . . . . 85

5.5 Discussion . . . . . . . . . . . . . . . . . . . . 88

5.5.1 Muscle Contractile Property . . . . . . . . . . . . . . . . 88

5.5.2 Fusion Index . . . . . . . . . . . . . . . . . . . . . . . . 89

6 Conclusion and Future Studies $\quad 95$

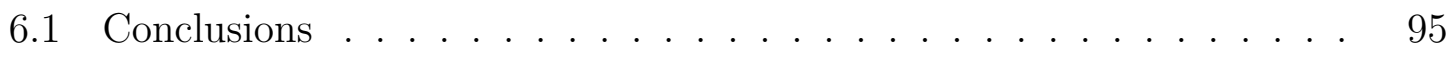

6.2 Future Study . . . . . . . . . . . . . . . . . . . . . . 98

$\begin{array}{ll}\text { Appendices } & 101\end{array}$

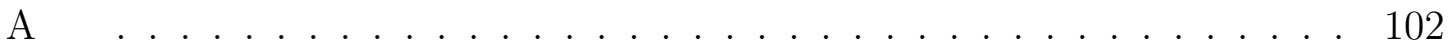

A.1 Quadrature Demodulation .............. 102

A.2 Laser Displacement Sensor . . . . . . . . . . . . . . . 105 
A.3 Triceps Brachii Lateral Head Muscle Monitoring . . . . . . . . 107

A.4 Angular Misalignment . . . . . . . . . . . . . . . . . 109

A.5 Figures Permission _................. 110

References 


\section{List of Figures}

2.1 Structure of sacromere and the sliding filaments (thick filament and thin filament). Image is reproduced from "Anatomy \& Physiology" by L. M. Biga, et al and the image is licensed under Creative Commons Atttribution 4.0 Unported license. Image is free access online at https://open.oregonstate.education/aandp/chapter/10-

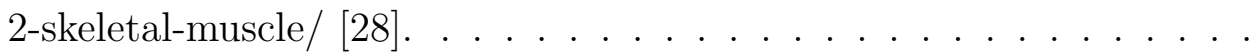

2.2 Process of myosin and actin cycling during muscle contraction. Image is reproduced from "Sliding filament theory of muscle contraction" by G. Karki and the image is licensed under Creative Commons Attribution 4.0 Unported license. Image is free access online at https://www.onlinebiologynotes.com/sliding-filament-modelof-muscle-contraction/ [32]. . . . . . . . . . . . . .

2.3 A typical single muscle twitch myogram. Image is reproduced from "Skeletal muscle" by Openstax College and the image is licensed under Creative Commons Attribution 3.0 Unported license. Image is free access online at https://commons.wikimedia.org/wiki/File:1012Muscle-Twitch-Myogram.jpg [36] . . . . . . . . . . . . 
2.4 Myograms of muscle reacting to different stimulation frequencies. Image is reproduced from "Sliding filament theory of muscle contraction" by G. Karki and the image is licensed under Creative Commons Attribution 4.0 Unported license. Image is free access online at https://www.onlinebiologynotes.com/sliding-filament-modelof-muscle-contraction/ [37]. . . . . . . . . . . . . . . . 12

2.5 A sample of fusion index frequency curve (a) and contractile parameters used in calculating the fusion index (b). The images are produced by S. Watanabe, et al. from "Estimating the minimum stimulation frequency necessary to evoke tetanic progression based on muscle twitch parameters". Physiological Measurement, Volume 38, Number 3, pp. 466-476, 7 February 2017. (C) Institute of Physics and Engineering in Medicine. Reproduced by permission of IOP Publishing. All rights reserved $[44] . \ldots \ldots \ldots \ldots \ldots \ldots \ldots \ldots \ldots$

2.6 Myograms of muscle reacting to a single electrical stimulus. The images are produced by S. Watanabe, et al. from "Estimating the minimum stimulation frequency necessary to evoke tetanic progression based on muscle twitch parameters". Physiological Measurement, Volume 38, Number 3, pp. 466-476, 7 February 2017. (C) Institute of Physics and Engineering in Medicine. Reproduced by permission of IOP Publishing. All rights reserved $[44] . \ldots \ldots \ldots \ldots \ldots$

2.7 Diagram of a pulse-echo ultrasonic measurement and the corresponding RF signal. . . . . . . . . . . . . . . . 
2.8 A hand-held conventional ultrasonic probe. Image is reproduced from "Medical Ultrasound linear array Probe/scan head/transducer" by D. W. Rickey and the image is licensed under Creative Commons Attribution-Share Alike 2.5 Generic license. Image is free access online at https://commons.wikimedia.org/wiki/File:UltrasoundProbe2006a.jpg

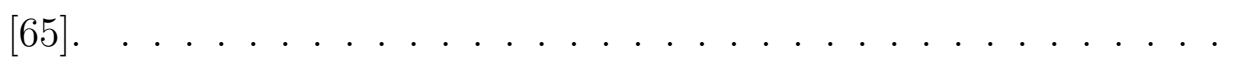

3.1 Schematic representation of the WUS (a) and a photograph of the WUS $(b) \ldots \ldots \ldots \ldots \ldots \ldots \ldots \ldots$

3.2 The near-field and far-field demonstrations. The image is reproduced by the author of this thesis based on the information discussed in "Diagnostic Ultrasound: Physics and Equipment" by P. Hoskins, et al. $[77]$

3.3 Ultrasound Measurement System . . . . . . . . . . . . . . . . . 31

3.4 The operation of the moving averaging on the ultrasonic RF signals. . 33

3.5 (a) The reference ultrasonic echo in red and the shifted ultrasonic echo in blue and (b) the correlation coefficient of the two signals. . . . . .

4.1 Photographs of the cylinder phantom (a) and the rectangular phantom (b)

4.2 Measurement configuration and procedure of phantom thickness estimation using LDS. . . . . . . . . . . . . . . . . . .

4.3 An experimental configuration for accuracy evaluation of the thickness estimation using a soft tissue-mimicking phantom and LDS as a golden standard. 
4.4 The raw RF signal obtained from the cylinder shaped phantom (a) and rectangular shaped phantom (b). . . . . . . . . . . . . . 47

4.5 The experimental configuration of the LDS in the phantom experiment. 50

4.6 An image of the manual squeeze phantom experiment with the cylinder

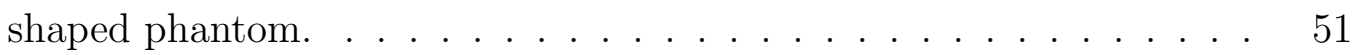

4.7 Phantom thickness changes caused by fingers squeeze calculated by peak detection (a), cross-correlation (b), quadrature demodulation (c) and LDS measurement $(\mathrm{d}) . \ldots \ldots 53$

4.8 A photograph of the servo motor used in the mechanical simulation. . 56

4.9 The phantom experiment configuration for the mechanical simulation. 56

4.10 The phantom experiment configuration for the muscle contraction evoked by EMS. . . . . . . . . . . . . . . . . . . . . 57

4.11 Muscle thickness measured by ultrasound measurement obtained from a tricep muscle electrically evoked at $14 \mathrm{~Hz}$. . . . . . . . . . . . . 59

4.12 Procedure of the angular position setting of the rotation arm in high EMS frequency muscle contraction simulation. . . . . . . . . . . 60

4.13 Phantom thickness changes estimated by LDS and ultrasound phase tracking method evoked by $2 \mathrm{~Hz}$ (a) and $10 \mathrm{~Hz}$ (b) motor rotational

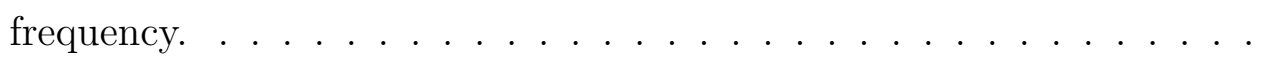

4.14 Thickness changes on stationary phantom estimated by the WUS measurement System. 1000 data points are analyzed from each muscle

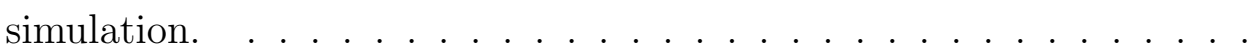

4.15 The observed environmental motion during muscle contraction simulation \#3 (2 Hz rotational frequency). . . . . . . . . . . . . . . . . 64

4.16 Absolute error calculated from muscle contraction simulation \#3 (2 Hz rotational frequency). . . . . . . . . . . . . . . . . . 
4.17 Enlarged Portion of Figure 4.14 (a) . . . . . . . . . . . 66

4.18 Absolute error obtained from various frequencies (0.5 to $12 \mathrm{~Hz}$ ) muscle contraction simulation in unfused tetanus period. 8000 points are analyzed from each muscle simulation. . . . . . . . . . . . . 68

4.19 Enlarged Phantom thickness changes estimated by LDS and ultrasound phase tracking method evoked at $12 \mathrm{~Hz}$ motor rotational frequency. .

5.1 In-vivo experiment setup for forearm muscle monitoring using WUS in pulse-echo mode. . . . . . . . . . . . . . . . .

5.2 Raw RF signal obtained from the forearm monitoring experiment with the indication of ultrasound pulse reflected from the ulnar bone. . . 72

5.3 The resultant RF signal after moving averaging with the frame numbers

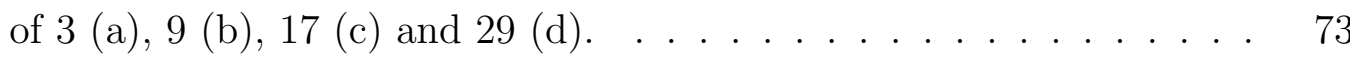

5.4 The SNR of the RF ultrasound pulse in Figure 5.2 with respect to the frame numbers of moving averaging. The SNR in experiment and in theory are shown by the blue cross marks and the red curve, respectively. 74

5.5 The resultant RF signal after moving averaging with the window size of raw (a), 3 (b), 9 (c) and 45 (d) ms. . . . . . . . . . 75

5.6 The relationship between RMSE and the various window sizes (frames). 76

5.7 The relationship between SNR and the RMSE. . . . . . . . . . . . 77

5.8 An example of the EMS pulse waveform. . . . . . . . . . . . 78

5.9 An example of the monophasic (left) and biphasic (right) EMS pulse waveforms. . . . . . . . . . . . . . . . . . . . 79

5.10 EMS 7500 device is shown on the left and an example EMS pulse waveform is shown on the right. . . . . . . . . . . . . 81 
5.11 The configuration circuit in measuring the EMS voltage across a 500 ohm resistor. . . . . . . . . . . . . . . . .

5.12 The triceps muscle antonym. Image is reproduced from "File:Triceps brachii muscle06.png" by Anatomography and the image is licensed under Creative Commons BY-SA 2.1 jp license. Image is free access online at https://commons.wikimedia.org/wiki/File:Triceps_brachii_muscle06.png $[126]$

5.13 Photograph of WUS and EMS electrodes attached to the skin surface of the arm for monitoring tetanus contraction of TBLH in pulse-echo mode. . . . . . . . . . . . . . . . . .

5.14 Subject 1 TBLH tissue displacement due to muscle contraction evoked by EMS (from pulse repetition frequency $2 \mathrm{~Hz}$ to $30 \mathrm{~Hz}$ ). . . . . . . . 86

5.15 Muscle motion observed without any electrical stimulation. . . . . . 87

5.16 Tissue displacement observed and muscle contractile properties extracted from a single twitch due to muscle contraction evoked by EMS at $2 \mathrm{~Hz} . \ldots \ldots \ldots$. . . . . . . . . . . . . . . 89

5.17 An example of fusion index calculation. . . . . . . . . . . . . . 90

5.18 The fusion index frequency curve (FFC) obtained from each subject. $\quad 91$

5.19 The relationships of unfused and fused frequencies obtained from the FFC and from contractile parameters of a single twitch at $2 \mathrm{~Hz}$ EMS

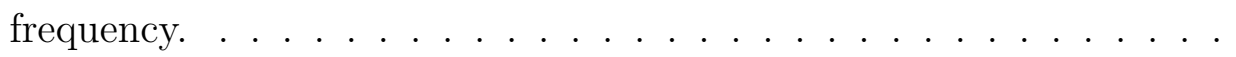

5.20 The relationship between the contraction time and fusion frequency of the TBLH predicted from the FFC. . . . . . . . . . . . . . . 94

1 The flow chart of the quadrature demodulation. . . . . . . . . . . . . 104 
2 Measurement principle and configuration of the laser displacement sensor.106

3 The reference distance and the measurement range of the laser displacement sensor (LK-H055, Keyence, Japan). . . . . . . . . . . . . . 106

4 A typical laser waveform obtained from the LDS receiver. . . . . . . . 107

$5 \quad$ Subject 2 TBLH tissue displacement due to muscle contraction evoked by EMS (from pulse repetition frequency $2 \mathrm{~Hz}$ to $18 \mathrm{~Hz}$ ) . . . . . . . 108

6 Subject 3 TBLH tissue displacement due to muscle contraction evoked by EMS (from pulse repetition frequency $2 \mathrm{~Hz}$ to $18 \mathrm{~Hz}$ ). . . . . . . 109

$7 \quad$ Example of an angular misalignment between the ultrasonic sensor and the boundary of a medium. . . . . . . . . . . . . . . . . . . 110

8 Permission granted from IOP Publishing to use the figures from "Estimate the minimum stimulation frequency necessary to evoke tetanic progression based on muscle twitch parameters" by S. Watanabe, et al. 111 


\section{List of Tables}

2.1 Sound velocity in various mediums. The data obtains from A. B. Wolbarst, Physics of Radiology. Appleton and Lange, 1993 [61]. . . . . . 21

3.1 Acoustical properties of several piezoelectric materials [60-62]. . . . . 28

3.2 The near-field distance corresponding to various sizes of the ultrasonic

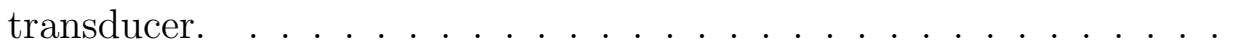

4.1 Acoustic properties of the gelatin-based materials and biological tissues

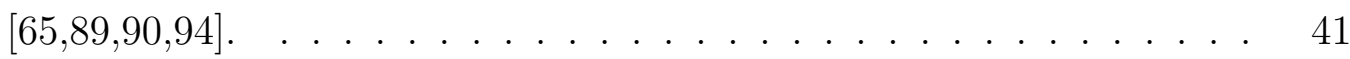

4.2 The composition and materials used in the gelatin-based phantom. . . 43

4.3 Acoustic properties of the gelatin-based materials and biological tissues

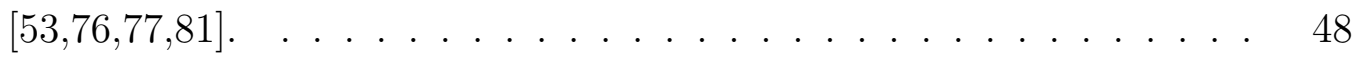

4.4 The comparison between each displacement estimation methods. . . . 54

4.5 Angular position and angular velocity setting for the servo motor in low EMS frequency muscle contraction simulation . . . . . . . 58

4.6 Angular position and angular velocity setting for the servo motor in high EMS frequency muscle contraction simulation. . . . . . . . . .

4.7 The RMSE and maximum absolute error observed from each phantom simulation experiment when the phantom is stable. 800 points are analyzed from each muscle simulation. . . . . . . . . . . 
4.8 The RMSE and maximum absolute error observed from each phantom simulation experiment during unfused tetanus period. 8000 points are analyzed from each muscle simulation. . . . . . . . . . . . 67

5.1 The health conditions of the subjects. . . . . . . . . . . . 81

5.2 EMS 7500 output voltage in various power levels measured with 500 ohms resistance. . . . . . . . . . . . . . . . . . 81

5.3 The experimental setup for the EMS device in each subject. . . . . . 82

5.4 Average initial thickness of the triceps brachii lateral head muscle for the three subjects. . . . . . . . . . . . . . . . . . . 88

5.5 The contractile properties extracted from tissue displacement evoked by $2 \mathrm{~Hz}$ EMS frequency. . . . . . . . . . . . . . . . . . . 90

5.6 Comparison of the unfused and fused frequencies estimated from the FFC and from contractile parameters of a single twitch at $2 \mathrm{~Hz}$ EMS frequency. . . . . . . . . . . . . . . . . 


\section{List of Abbreviations}

C/C Cross Correlation

EMG Electromyogram

FCU Flexor Carpi Ulnaris

FFC Fusion Index Frequency Curve

FI Fusion Index

LDS Laser Displacement Sensor

MMG Mechanomyography

MRI Magnetic Resonance Imaging

P/D Peak Detection

PVDF Polyvinylidene Fluoride

PZT Ziroconate Titanate

Q/D Quadrature Demodulation

RF Radio Frequency 
RT Muscle Relaxation Time

sEMG Surface Electromyogram

SNR Signal-to-noise signal

TBLH Triceps Brachii Lateral Head

Tc Contraction Time

Th Maximum Contraction Displacement

TMG Tensiomyography

TOF Time of Flight

V Ultrasound Velocity in the medium

Vc Contraction Velocity

wt\% Percentage in Weight

WUS Wearable Ultrasonic Sensor

\section{Nomenclature}

$\Delta d(n)$ Thickness changes of the medium

$\Delta L \quad$ Change in length of phantom due to a mechanical force

$\Delta w \quad$ Downshift of the echo frequnecy 
$\Delta \mathrm{t}$ The time delay between the first round trip echo and the second round trip echo

$\mu \quad$ Attenuation coefficient

$\rho \quad$ Density of the material

$\rho_{p} \quad$ Density of the phantom

$\theta \quad$ Instantaneous phase of the $\mathrm{RF}$ signal

A Amplitude of the received ultrasonic signal

a Number of frame averaging

$A_{S} \quad$ Peak to peak amplitude of the ultrasound echo of interest

Ao Original ultrasonic signal strength

$A p_{1} \quad$ Peak to peak amplitude of the ultrasound echo completed the first round trip

$A p_{2} \quad$ Peak to peak amplitude of the ultrasound echo completed the first round trip

$D \quad$ Length of the ultrasound transmitter

$d_{o} \quad$ Initial thickness of the phantom

$d_{t} \quad$ Distance of the ultrasound pulse travel

E Reference echo signal

$f_{c} \quad$ Center frequency of the transmitting ultrasound

$\mathrm{Fr} \quad$ Rotational frequency of the servo motor 
G Correlation Coefficient

$H \quad$ Consecutive echo signal

$I(n)$ In-phase component of the quadrature demodulator

$k \quad$ Total number of frames

$L P F$ Low pass filter

$M \quad$ Volume of the phantom

$N \quad$ Near-field zone of a transducer with a square active area

$n \quad$ Sample Index

$Q(n)$ Quadrature component of the quadrature demodulator

$R \quad$ Reflection coefficient

$R M S$ (Noise) Root mean square of the noise within a RF signal

$R M S E$ Root mean square error

$R T_{10 \%}$ Time duration from the peak of the muscle twitch to $10 \%$ normalized force

$R T_{90 \%}$ Time duration from the peak of the muscle twitch to $90 \%$ normalized force

$S \quad$ Number of transaction steps of the servo motor

$t \quad$ Time taken for the ultrasound pulse to travel within the medium

Ts Sampling time 
$v \quad$ Ultrasound velocity in the medium

$v_{h} \quad$ Average ultrasound velocity in human soft tissue

vm Maximum speed of the thickness changes measured by phase tracking method

W Weight of the phantom

w Angular Velocity

Z Acoustic impedance

$Z \quad$ acoustic impedance

Dg Distance between the LDS head and phantom

Dp Distance between the LDS head and PMMA plate

$\mathrm{v}_{p} \quad$ Ultrasound velocity within the phantom 


\section{Chapter 1}

\section{Introduction}

\subsection{Overview}

The skeletal muscle has an important role in daily life. By means of contracting and relaxing the muscle, actions such as jogging and facial expressions can occur. Monitoring the motion of the skeletal muscle provides valuable information about the healthiness of the muscle. The quantitative assessment of the muscle function can be applied in the areas of rehabilitation, physical training, diagnosis of muscular disorders, mechanics of neurophysiology and muscle elasticity [1-5]. Monitoring muscle behaviour is also of great interest to the sports industry for muscle functional performance (eg. running speed, jumping, etc.) $[6,7]$ and evaluate the effectiveness of the training $[3,5,8]$.

Various measurement techniques have been proposed for monitoring skeletal muscle contraction including electromyography, mechanomyogram, MRI and ultrasonography. However, the current techniques have several limitations, including the dynamics analysis only provide a net output of tissue activities by all surrounding muscles around a joint [9]. Furthermore, the motion of the body may cause motion artifacts, 
which may result in measurement error [10]. Among these technologies, ultrasound technology is capable of penetrating through the skin to measure the thickness information of the skeletal muscle and the surrounding tissues $[11,12]$. It provides real-time information for monitoring skeletal muscle contraction.

\subsection{Problem Statement}

A conventional handheld ultrasonic imaging probe is typically bulky and heavy. Therefore, it may be difficult to secure the probe in position with respect to the skin surface to obtain an accurate signal acquisition, especially if the tissue of interest is in motion, such as muscle contraction. The physical size of the probe may also restrict the activity of the muscle of interest [10]. Furthermore, the inconsistent probe movement may cause measurement error [13]. Hence, such a probe may not be suitable or applicable for long-term continuous muscle monitoring during muscle contraction and relaxation. In our previous studies, a wearable ultrasound sensor (WUS) has been developed for tissue monitoring [5,13-15]. The flexibility, lightweight and compact size of the WUS are suitable features for the continuous monitoring of the tissue motion and reduce undesired motion artifacts in the measurements.

The skeletal muscle is considered as a near-constant volume system in which the muscle shortening due to muscle contraction is related to the increase in muscle thickness $[8,16]$. Since the WUS is able to measure the thickness change of the tissue due to muscle contraction, the muscle activities can be monitored [17]. However, the reliability of the WUS measurement system is yet to be confirmed. Since the accuracy of the thickness measurement is also related to the signal processing methods, it is important to choose an appropriate signal processing method. To ensure the accuracy and repeatability of the WUS measurement system, it is required to compare the 
thickness measurement results by the WUS with those obtained by a golden standard method.

\section{$1.3 \quad$ Objective}

The primary objective of this thesis is to develop a reliable technique to characterize skeletal muscle contractile properties in ultrasound pulse-echo mode using a WUS, of which has been designed and constructed by our research group.

One of the goals of this thesis research is to determine the repeatability and accuracy of the WUS measurement system to measure the thickness changes of the tissue during muscle contraction. Another objective is to determine which signal analysis technique for thickness change measurements could yield more accurate results via the WUS. In order to investigate the repeatability and accuracy of the WUS measurement system, an experimental environment with a soft tissue-mimicking phantom and mechanical stimulation system are developed in order to compare the thickness measurements with those done by a laser displacement sensor (LDS) as a golden standard.

Finally, in-vivo experiments using the WUS are performed to characterize muscle contractile properties with electromyostimulation (EMS). Since human tissues are more complicated than that of the phantom, more signal attenuation occurs in the in-vivo experiment. Therefore, it is expected to research pre-processing techniques to improve the signal-to-noise ratio and the tissue thickness measurement accuracy. The ultimate goal of this research is to achieve a continuous monitoring of muscle movement to study the characteristics of the skeletal muscles using the proposed WUS measurement system. 


\subsection{Thesis Contributions}

The following is a list of the main research contributions demonstrated in this thesis:

- Develop both software and soft tissue mimicking phantom simulation environments to perform ultrasonic thickness measurement.

- Develop a mechanical system to simulate the EMS muscle contraction.

- Evaluation of the thickness measurement methods including peak detection, cross-correlation, and phase tracking method.

- Evaluate the repeatability and accuracy of the ultrasonic thickness measurement and the developed thickness changes measurement method by comparing with the laser displacement sensor.

- Perform muscle monitoring on a voluntary isometric contraction and EMS evoked muscle contraction.

- Investigate the relationship between the signal-to-noise ratio and the measurement error of the WUS measurement system.

- Develop and demonstrate a method for characterizing muscle contractile properties from continuous ultrasonic measurements of skeletal muscle contraction evoked by the EMS.

- Estimate the minimum EMS evoked unfused and fused tetanic contraction frequencies from a fusion index frequency curve and a single $2 \mathrm{~Hz}$ EMS frequency. 


\subsection{Research Publications}

- Elliot Yeung, Ibrahim AlMohimeed, and Yuu Ono "Estimation of Tissue Thickness Changes Due to Electrical Muscle Stimulation Using Wearable Ultrasonic Sensor in Pulse Echo Mode," 2019 IEEE SENSORS, Montreal, QC, Canada, 2019, pp. 1-4.

\subsection{Thesis Organization}

Chapter 1: The introduction of the thesis including the objectives, problem statement and the contributions.

Chapter 2: Background information in human skeletal muscle physiology, microarchitecture of the muscle fiber, various muscle types and current methods in muscle monitoring.

Chapter 3: Description of the WUS and the signal processing techniques applied for measuring tissue thickness changes.

Chapter 4: Evaluate the thickness measurement techniques by comparing with a golden standard and then evaluate the repeatability and accuracy of the WUS in thickness measurements.

Chapter 5: Perform voluntary muscle contraction monitoring on the forearm muscle and then evaluate the improvement of signal-to-noise ratio after performing moving averaging filter. Then, the thickness changes of the triceps muscle evoked by EMS are performed on three human volunteers.

Chapter 6: A summary of this thesis and the recommendations for future studies. 


\section{Chapter 2}

\section{Background Review}

In this thesis work, the electrical and mechanical properties of the skeletal muscle are being investigated using ultrasound. This chapter provides an introduction to human muscle physiology and skeletal muscle properties. Then, the current noninvasive methods of measuring muscle behaviors are discussed. Finally, the principle and theory of ultrasound for measuring tissue thickness is presented.

\subsection{Human Muscle Physiology}

Muscle is a soft tissue which produces mechanical force, motion and heat for multiple purposes. There are three types of muscles: skeletal, smooth and cardiac muscles [18]. Skeletal muscle connects the bones and generate the bone movement by contraction. Voluntary muscle is also known as skeletal muscle, due to the fact that the muscle is controlled by a voluntary nervous system $[19,20]$. Smooth muscle generates pressure to internal organs and blood vessels [18]. Cardiac muscle can only be found in the heart and at the end of the main blood vessels to aid the circulation system [18]. Both smooth and cardiac muscles, which are controlled by the autonomic nervous system, 
regulates the basic internal body functionality, including breathing and digestion [21]. As the combination of the muscle motors differ between muscle types, smooth and cardiac muscles can generally apply long periods of contraction without fatigue. In contrast, skeletal muscle experience fatigue in shorter periods of time [22]. The muscle fiber types will be discussed in section 2.2.3. Skeletal muscle contraction monitoring has gained interest for many medical applications such as follow-up the recovery of the muscle after an injury [1,23] and used in neuromuscular disorder diagnosis $[5,24]$. In the sport industry, the muscle monitoring is used to evaluate the effectiveness of training $[25,26]$ and determining the muscle fiber types for muscle performance improvement [27]. This study focuses on the contractile properties of the skeletal muscles. A brief introduction on muscle mechanisms and muscle contraction will be provided in the following sections.

\subsection{Skeletal Muscle Contraction and Relaxation}

Skeletal muscle is a complex structure which is made by multiple types of tissues. These tissues include muscle fibers, blood vessels, nerve fibers, and connective tissues. The functional unit of the contraction is called sarcomere. The structure of the sarcomere is formed by the interaction between two types of myofibrils (thick and thin filaments) shown in Figure 2.1 [28]. The thick filaments have more mass and appear thicker due to the myosin heads pointed outward from the center of the filament. The thin filament is made from a chain of actin subunits and troponin complex. Each actin subunit contains a myosin binding site and allows the myosin to hold on to it. The sliding filament is a theory that involves the interaction between the two filaments, which generate a mechanical force to shorten the length of the sarcomere $[29,30]$.

Myosin-actin cycling is the process of the muscle contraction where myosin pulls 


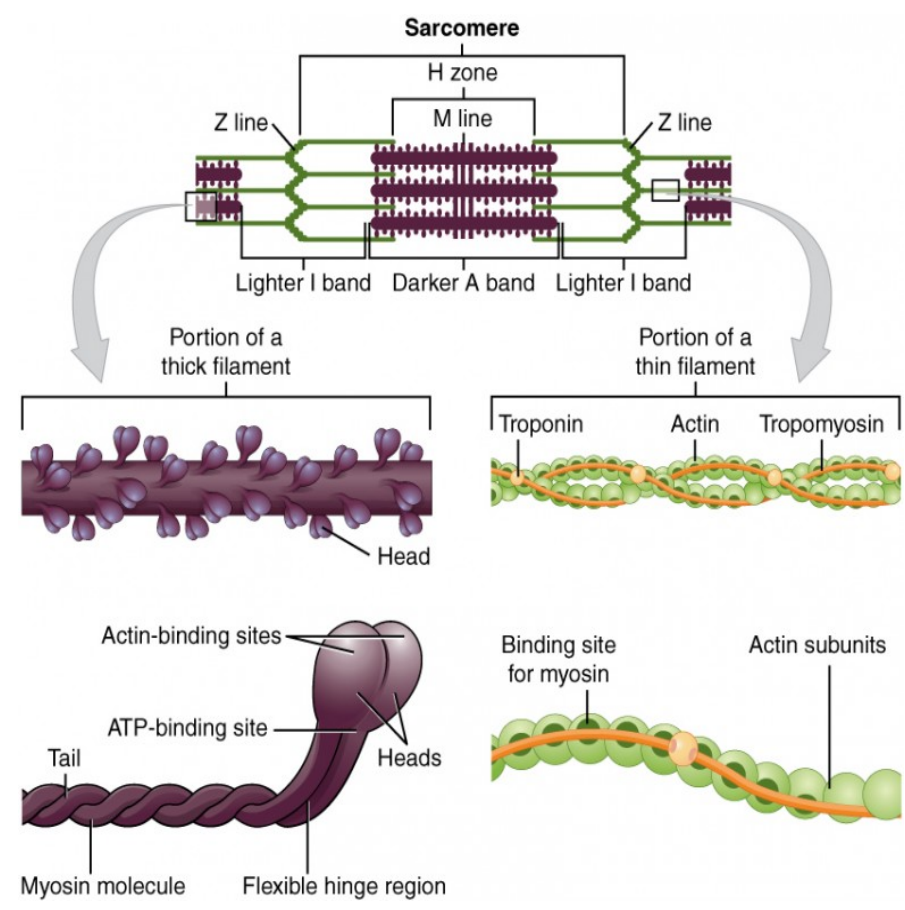

Figure 2.1: Structure of sacromere and the sliding filaments (thick filament and thin filament). Image is reproduced from "Anatomy \& Physiology" by L. M. Biga, et al and the image is licensed under Creative Commons Attribution 4.0 Unported license. Image is free access online at https://open.oregonstate.education/aandp/chapter/10-2-skeletalmuscle/ [28].

the actin [31]. The full myosin-actin cycle is shown in Figure 2.2 [32]. Assume the cycling begins when the cross bridge between the myosin and actin binding site is formed. 1. Myosin head binds with ATP which detaches the myosin from the actin binding site. 2. The attached ATP hydrolysis into ADP and inorganic phosphate which supply energy for a structural change on the myosin head. 3. The release of inorganic phosphate allow the myosin head to attach to the actin, forming a new cross bridge. 4. The release of the ADP allow the myosin to return to its original position and pull the actin along with it. The pulling of the actin shortens the length of the sarcomere and causes the muscle contraction. The myosin-actin cycling is continued when the muscle fiber is surrounded by enough amount of ATP and stimulus [33]. 
However, without the presence of calcium ion, the actin binding sites are blocked by the troponin-tropomyosin complex, which prevents the myosin heads from binding with the actin from the thin filament, and thus preventing the process of myosinactin cycling [18]. The release of calcium ion requires a series of interactions between the neurons and the surface of muscle fibers [30].

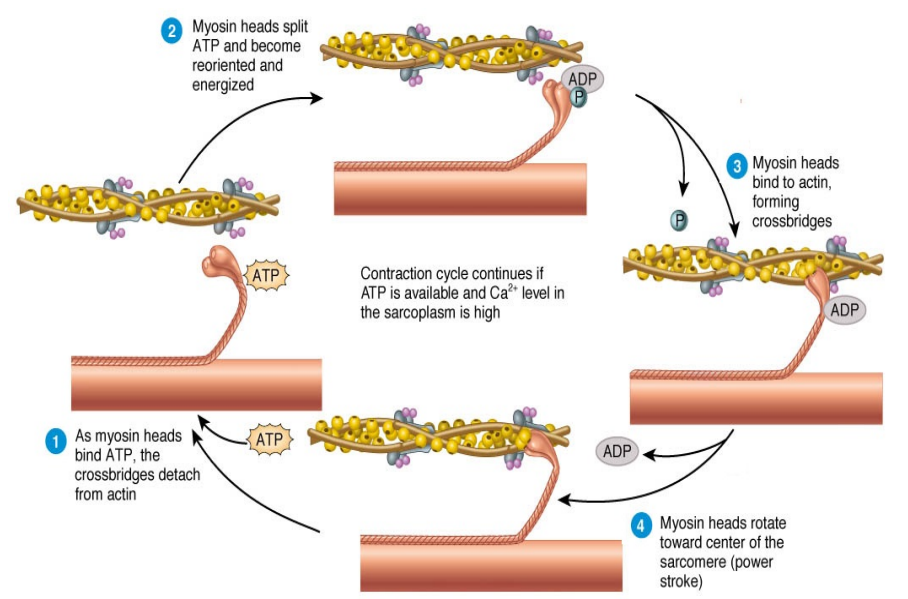

Figure 2.2: $\quad$ Process of myosin and actin cycling during muscle contraction. Image is reproduced from "Sliding filament theory of muscle contraction" by G. Karki and the image is licensed under Creative Commons Attribution 4.0 Unported license. Image is free access online at https://www.onlinebiologynotes.com/sliding-filament-model-of-musclecontraction/ [32].

\subsubsection{Electrical Stimulus in Muscle Contraction}

Both neurons and muscle cells are electrically excitable and hence, the muscle fiber is contracted only when an electrical stimulus arrives at the muscle. In voluntary contraction, the initial stimulus is an action potential sent by the central nervous system through the neurons. The neuron which connects the muscles is called motor neuron [18]. When the signal reaches to the end of the motor neuron, the motor neurons release the chemical signal - the neurotransmitter to the muscle fiber. The release of neurotransmitter is then bound with the receptors on the surface of the 
muscle fiber, which causes the release of sodium ion. These positive charged ions depolarize the membrane of the fiber and action potential is generated. The action potential triggers the release of the calcium ion which is stored in the sarcoplasmic reticulum. The calcium ion will bind to the troponin and physically change the shape of the troponin-tropomyosin complex [34]. The myosin head from the thick filament will bind immediately with the actin binding site since the binding site is no longer blocked by the troponin-tropomyosin complex and continue the process of myosinactin cycling. On the other hand, when the electrical stimulus is absent, the calcium ion will not be released by the sarcoplasmic reticulum and the ion pumps on the reticulum will pump out the calcium from the muscle. As a result, the calcium level within the muscle decreases and the troponin-tropomyosin complex will return to its original shape and block the actin binding site $[33,34]$. Hence, the myosin head cannot bind with actin to form a cross bridge, the sarcomere slowly losing strength and return to its original length.

\subsubsection{Single Muscle Twitch and Fused Muscle Contraction}

Usually multiple muscle fibers connect with one motor neuron which forms a single motor unit. The number of muscle fibers in a single motor unit varies from the functionality of the muscle [35]. For example, motion units with less muscle fibers allow better control of the movement but lesser force. In comparison, motor unit with more fiber units provide a greater force but coarse movement. A single muscle twitch occurs when an action potential travels down the motor neuron and all the muscle units connected with the same motor neuron contract simultaneously. Figure 2.3 shows the full cycle of a single muscle twitch when a single stimulus is received [36]. The single muscle twitch is composed of three periods: latent period, contraction period and relaxation period [36]. The latent period is the delay when the stimulus 
is received by the muscle, but the muscle is yet to respond. This delay is related to the release of the calcium ion from the sarcoplasmic reticulum and the time for the calcium binding with the troponin [18]. The contraction period is the time between the beginning and end of the muscle contraction where it reaches its maximum length. This corresponds to the process of myosin-actin cycling. The relaxation period is the time between the muscle length decline until the muscle returns to its normal length. The calcium level within the muscle membrane is maintained by a calcium ion pump, which continuously removes calcium from the inner membrane. Since the rate of the calcium ions pumped away from the membrane is slower than the sarcomere reticulum being released into the membrane, the full relaxation period is longer than the contraction period.

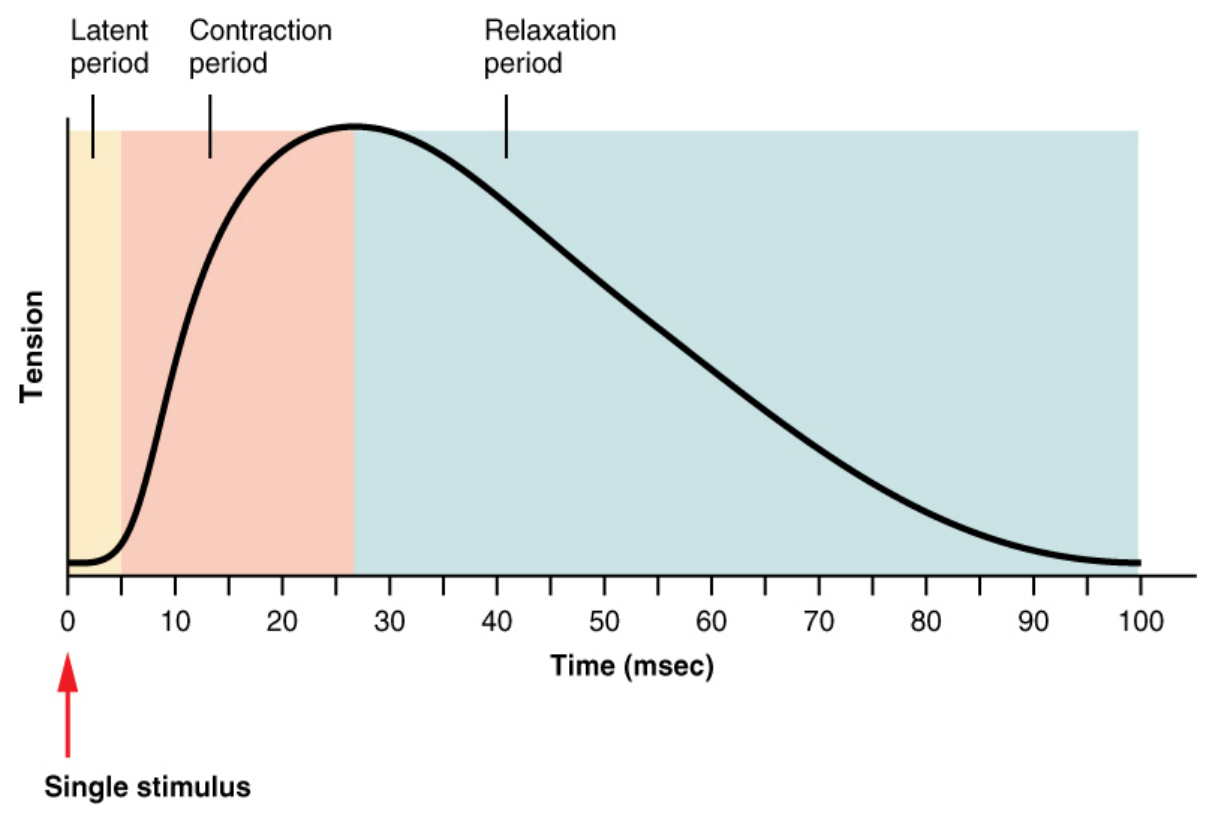

Figure 2.3: A typical single muscle twitch myogram. Image is reproduced from "Skeletal muscle" by Openstax College and the image is licensed under Creative Commons Attribution 3.0 Unported license. Image is free access online at https://commons.wikimedia.org/wiki/File:1012-Muscle-Twitch-Myogram.jpg [36]

Figure 2.4 shows a tension waveform of a typical muscle reaction to a series of 
electrical stimulation in varying frequencies [37]. Wave summation happens when the stimulus firing rate is higher than the duration of a single twitch. Due to the fact that muscles cannot relax completely, the muscle tension gradually increases and causes unfused tetanus [38]. In general, the central nervous system uses wave summation to increase the contractile strength. Fused tetanus contraction is the situation when it is difficult to differentiate the contraction and relaxation period [18]. The rate of stimulation that produce fused tetanus contraction is called fusion frequency. It is varies with muscle fiber types and body temperature [39].

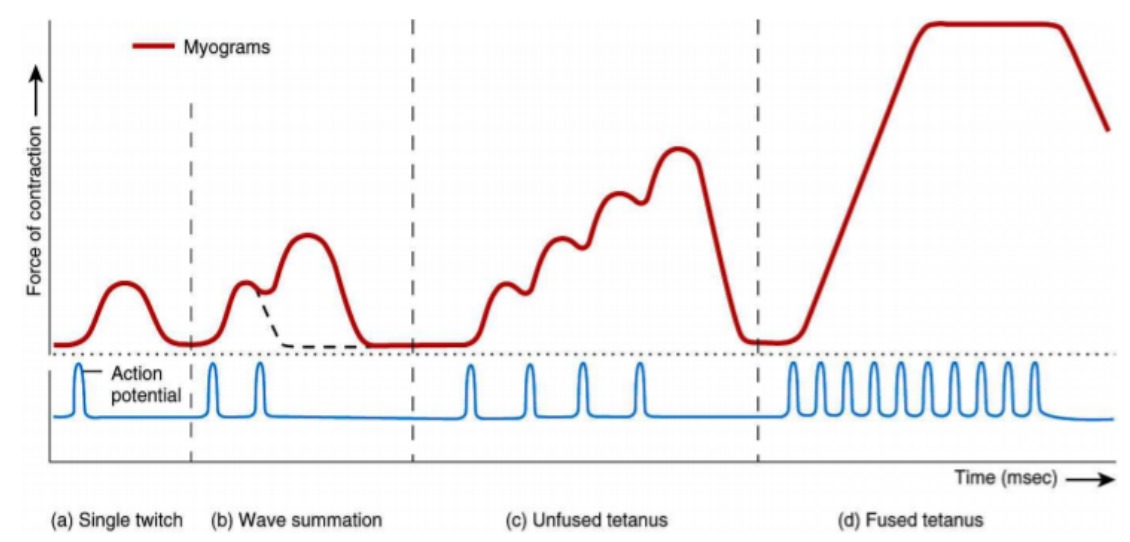

Figure 2.4: Myograms of muscle reacting to different stimulation frequencies. Image is reproduced from "Sliding filament theory of muscle contraction" by G. Karki and the image is licensed under Creative Commons Attribution 4.0 Unported license. Image is free access online at https://www.onlinebiologynotes.com/sliding-filament-model-of-musclecontraction/ [37].

\subsubsection{Muscle Fiber Types}

In human skeletal muscle, there are two types of muscle fibers and they are called fast twitch fibers and slow twitch fibers $[38,40]$. Motor units with different combination of the two fibers affect the contractile properties of the muscle. The fast twitching muscle fibers has a faster contraction speed which is related to a fast cross-bridge cycle. The 
myosin in fast twitching fibers have a fast ATPase which, they can hydrolyze ATP rapidly, increase the rate in myosin and actin cycling. In addition, the fast twitch muscle fiber has more efficient calcium ion pumps and the calcium ion is removed at a faster rate. These properties of fast twitch fibers cause a faster contraction and relaxation period when compared with the slow muscle. Therefore, the fast muscle fiber has a higher fusion frequency and allow for rapid muscle movement [38]. Another distinctive difference between the two muscle fiber types, is the metabolism in production of ATP. Glycolysis break down glucose and produce ATP [41]. Glycolysis takes place in the cytoplasm, where ATP production is not required for any specific organelle. The benefits of glycolytic pathways are that ATP can be produced instantly and oxygen is not required in the chemical reaction [41]. The fast twitching muscle needs the production of ATP in a short period of time. Therefore, glycolysis is the best option. Another metabolism in ATP production is oxidative respiration which occurs in mitochondria. In this pathway, oxygen is required for ATP generation and the ATP production is sufficient enough to supply energy for muscle cells for a long period. However, this pathway cannot produce ATP instantly; it is not used by the muscle that require instant power. The main ATP source of energy of slow muscle fibers comes from oxidative pathways. The abundance of mitochondria within the slow muscle allows a constant supply of ATP to maintain the muscle activity for a continuous duration. As a result, the slow muscle fibers are more resistant to fatigue [42].

\subsubsection{Fusion Index}

Previous studies have investigated the muscle contractile properties while evoking the muscle by an external electrical stimulation device. In 1930, Cooper finds that the minimum frequency evoked unfused and fused tetanus frequencies which are different 
from muscle to muscle [43]. The author also explains that the different muscle units have different contraction times and relaxation times, which can be the reasons behind the varied unfused and fused tetanus frequencies [43]. This theory is further supported by Celichowski, et al. [39]. They find that the slow muscle units have a low minimum unfused and fused tetanus frequencies in comparing with fast muscle units and it is possible to investigate the muscle fiber type based on the unfused/fused frequencies [39]. They further propose fusion index (FI) to evaluate tetanus progression. An example FI frequncy curve is shown in Figure 2.5 (a). FI at 0\% corresponds to single muscle twitch where the muscle can be fully relaxed. The FI at 100\% shows fused tetanus where there are no relaxation periods in the muscle. The FI is calculated by dividing the minimum value over the maximum value of force in each stimulating frequency as shown in Figure 2.5 (b) [44]. $\mathrm{F}_{10 \%}$ and $\mathrm{F}_{90 \%}$ are defined when the FI is at $10 \%$ and $90 \%$ [44]. The EMS evoked minimum unfused and fused tetanus frequencies can be evaluated from the FI at $10 \%$ and $90 \%$ as shown in Figure 2.5 (a) [39].

There is a drawback in creating the FI-frequency curve (FFC). Since the muscle has to be stimulated in multiple frequencies continuously, the muscle cannot attain full relaxation at high frequency and fatigue may occur before achieving fused tetanus. During muscle fatigue, the patients may experience physical discomfort and possibly pain. Additionally, muscle contractile properties, such as contraction distance, may change after experiencing muscle fatigue $[42,45]$. In order to prevent this issue, Watanabe, et al. proposed using the contraction time and relaxation time in a single muscle twitch to estimate the minimum EMS evoked unfused and fused tetanus frequencies [44]. Figure 2.6 shows the contractile properties obtained from a single twitch muscle myogram. Contraction time $(T c)$ is the time it takes for the muscle to contract to the maximum twitch. Relaxation time $(R T)$ is the time it takes for the muscle to fully relax. $R T_{10 \%}$ and $R T_{90 \%}$ are the time duration from the peak of the 


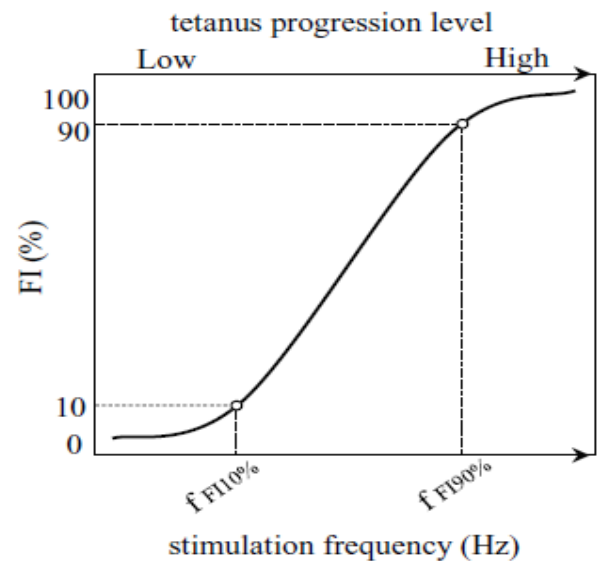

(a)

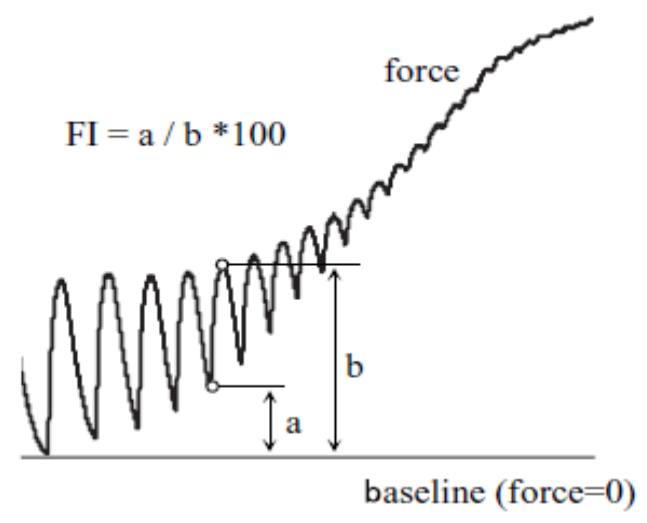

(b)

Figure 2.5: A sample of fusion index frequency curve (a) and contractile parameters used in calculating the fusion index (b). The images are produced by $S$. Watanabe, et al. from "Estimating the minimum stimulation frequency necessary to evoke tetanic progression based on muscle twitch parameters". Physiological Measurement, Volume 38, Number 3, pp. 466476, 7 February 2017. (C) Institute of Physics and Engineering in Medicine. Reproduced by permission of IOP Publishing. All rights reserved [44].

twitch to the time when the twitch force decreased to $10 \%$ normalized force and $90 \%$ normalized force, respectively. Watanabe, et al. conducted an animal experiment to measure the muscle forces evoked by EMS in various firing frequencies [44]. Muscles which are mainly composed of fast twitching muscle fiber, slow twitching muscle and combination of the two muscle fibers were the focus of their research [44]. Watanabe, et al proposed the minimum stimulation frequency for unfused tetanus can be estimated by

$$
F_{1}=\frac{1}{\left(T c+R T_{10 \%}\right)}
$$

and fused tetanus can be estimated by

$$
F_{2}=\frac{1}{\left(T c+R T_{90 \%}\right)}
$$




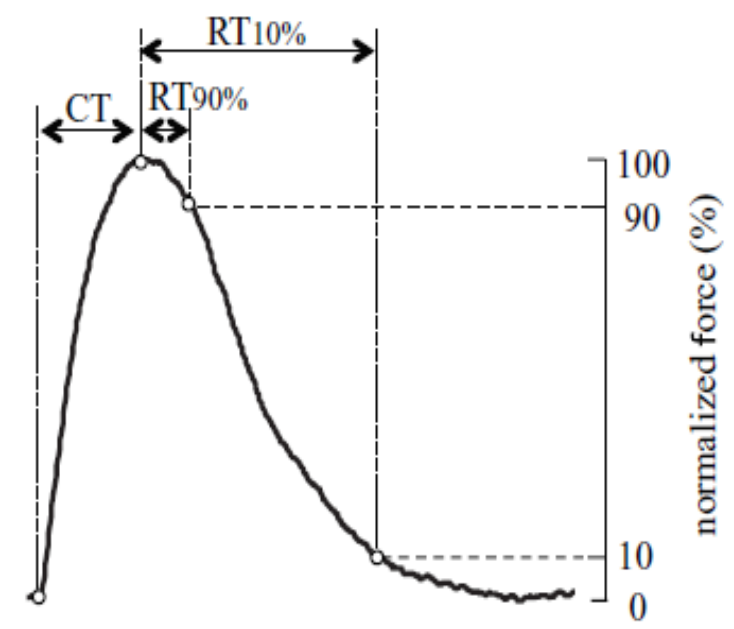

Figure 2.6: Myograms of muscle reacting to a single electrical stimulus. The images are produced by $S$. Watanabe, et al. from "Estimating the minimum stimulation frequency necessary to evoke tetanic progression based on muscle twitch parameters". Physiological Measurement, Volume 38, Number 3, pp. 466-476, 7 February 2017. (C) Institute of Physics and Engineering in Medicine. Reproduced by permission of IOP Publishing. All rights reserved [44].

Figure 2.5 (a, b) and Figure 2.6 are images produced by S. Watanabe, et al. and permission are granted to use and demonstrate the images in this thesis (Appendix A.5).

\subsection{Existing Methods of Monitoring Skeletal Muscle Behavior}

The valuable information obtained from skeletal monitoring can be used in applications such as physical training, strengthening, muscle pain, muscle illness, injury and rehabilitation. The measurement should be risk-free to the subject. Therefore, noninvasive muscle monitoring technologies are the focus in some studies $[8,9]$. There are many existing methods in measuring muscle properties including electromyogram 
(EMG), Mechanomyography (MMG), Magnetic Resonance Imaging (MRI) and ultrasonography. The following sections (2.3.1-2.3.4) provide a brief insight into the methods used in muscle measurement.

\subsubsection{Surface Electromyogram (sEMG)}

sEMG is one of the most used muscle monitoring measurement in evaluating the electrical activity produced by the skeletal muscle units and muscle neurons [46]. Bio-electrical activity of the individual muscles, which correlates with contractile force, can be measured by EMG [46]. EMG provides individual muscle's bio-physical activity, which also has been correlated with contractile force [9]. The sEMG receives the muscle neurons electrical signals by placing a conductive electrode on the surface of the skin.

When a greater force is needed, more motor units are recruited and the received sEMG signals will also increase. Therefore, the amplitude of the sEMG indicates the level of motor recruitment [47]. Several studies have investigated animal muscle fibers types compositions using sEMG [48-50]. During a single muscle contraction, muscle parameters such as muscle conduction velocity and median frequency can be analyzed from the sEMG signal. These parameters provide information of the muscle fiber type composition estimation [48-50]. There are a few major concerns in sEMG measurements and they are discussed as follow. The mechanical properties of the muscle are undetectable by sEMG. Since the sEMG collects the area of electrical activities during muscle contraction, it is difficult to investigate the properties of a unique muscle in a chosen depth [51]. Another study indicates that sEMG have accuracy issues when measuring muscle contraction at a high velocity [9]. It may be caused by the distance changes between the electrodes and muscle during the contraction. Additionally, the measurement condition (such as skin humidity and 
contact resistances) should remain constant during the measurement, in order to obtain an accurate result [9]. However, these measurement conditions are difficult to maintain for the entire measurement duration.

\subsubsection{Mechanomyography (MMG)}

During muscle contraction pressure waves are generated and the pressure generated evokes the mechanical changes. MMG records these mechanical motions in the purpose of understanding the particular muscle properties [52]. MMG related research demonstrate the relationship between the muscle dynamic changes and the MMG signals extracted parameters [53]. Currently, there are several types of MMG on the market, among which the accelerometer is one of the commonly used. The accelerometer-based MMG measures the movement and vibration of the tissue from the surface of the skin in terms of acceleration [53]. The MMG is able to record the motion from the surface of the skin and it provides the total motion underneath the sensor area. This causes difficulty to detect the motions from a specific muscle at a chosen depth under the skin [53]. Additionally, the motion artifacts and interference might induce into the MMG signals during muscle contraction. The other commonly used MMG available on the market is the laser displacement sensor (LDS) [54-56]. A brief introduction of the LDS is given in Appendix A.2.

The skeletal muscle is considered as a near-constant volume system in which the muscle shortening due to muscle contraction is related to the increase in muscle thickness [8]. Tensiomyography (TMG) is one of the modalities of MMG, which measures the displacement of the muscle during muscle contraction $[2,8]$. The TMG consist of a spring-loaded probe and a displacement sensor which is pressed on a fixed surface of the skin during muscle monitoring. Studies have indicated that the TMG is being used in the following applications, such as determination of muscle 
stiffness, rehabilitation, non-invasive muscle fiber composition estimation, treatment efficacy evaluation and muscle fatigue [1-4]. However, TMG muscle monitoring only allows the measurement of electrical stimulated isometric muscle contraction, and the voluntary muscle contraction are not included. The main challenge of the TMG is the difficulty in performing muscle monitoring during physical activities. Furthermore, the muscle activities are restricted due to the nature of the spring-loaded sensor.

\subsubsection{Magnetic Resonance Imaging (MRI)}

MRI image provides the functional images of the tissue of interest in a high-resolution perspective. It has become a useful tools in diagnosis and follow-up for patients with muscle dystrophies [57]. Due to the high cost of MRI scanning, it is barely used in monitoring muscle motions. Unlike other technologies, MRI monitoring must occur in a specific location such as laboratories or hospitals. Due to the restriction of the sensing area in an MRI, the muscles of interest might not be able to move freely. Therefore, it is difficult to measure the dynamic changes of the muscle movement in some cases [58].

\subsubsection{Ultrasonography}

Ultrasonography has been one of the most important non-invasive imaging method of the human body. It is able to provide some real-time, low cost and low risk images of the soft tissues [5]. Some studies have investigated the ability of ultrasound modality in detecting dynamic change of the deep muscle tissues during muscle contraction $[59,60]$. Muscle thickness changes before and after contraction can be observed from the ultrasound images [61].

A research study, conducted by Scott, et al., has evaluated the availability of 
ultrasonic technology in muscle mass monitoring [62]. The quadriceps and gastrocnemius muscles are monitored by using panoramic ultrasound. By comparing with MRI results, the study has concluded that ultrasound imaging is suitable in monitoring the atrophy of the quadriceps and the gastrocnemius muscle [62]. Furthermore, muscle fatigue during isometric contraction can be observed by ultrasound imaging [63]. Some studies also indicate that the types of muscle fiber composition can be identified by the muscle fatigue properties [64]. Additionally, muscle fatigue properties can be used as an indicator in evaluating the stage of muscle rehabilitation from an injury $[5]$.

\subsection{Ultrasound Basic}

Ultrasonography is an image modality obtained by using the properties of high frequency ultrasound. In medical ultrasound imaging, the sound wave frequency is from $1 \mathrm{MHz}$ to $10 \mathrm{MHz}$ [62]. The ultrasound pulses, generated by an ultrasonic transducer, are transmitted into the soft tissue. In pulse echo mode, the transmitted ultrasound pulses are reflected at tissue boundaries and returned back to the same transducer. The returned ultrasound converts into an electrical signal, which is also called radio frequency signal (RF signal). Ultrasound propagates though different mediums with various velocities, depending on the type of the medium. Table 2.1 shows the velocity of sound in different materials [65]. In medical ultrasound, $1540 \mathrm{~m} / \mathrm{s}$ is usually used for the average velocity of sound in soft tissues [66]. Hence, by measuring the timeof-flight of the ultrasound pulses in the RF signal, the thickness of the soft tissues can be estimated. Equation 2.3 shows the time-of-flight (TOF) equation in pulse echo mode [67]. 
Table 2.1: Sound velocity in various mediums. The data obtains from A. B. Wolbarst, Physics of Radiology. Appleton and Lange, 1993 [61].

\begin{tabular}{|c|c|c|c|c|c|c|c|}
\hline Medium & Air & Water & Bone, & Blood & Brain & Liver & Heart \\
\hline Sound velocity & $330-$ & $1480-$ & $3180-$ & $1564-$ & $1555-$ & $1590-$ & $1552-$ \\
$(\mathrm{m} / \mathrm{s})$ & 331 & 1498 & 3500 & 1586 & 1575 & 1618 & 1590 \\
\hline
\end{tabular}

$$
d_{t}=\frac{v \cdot t}{2}
$$

where $d_{t}$ is the distance between the ultrasound transducer and the boundary where the reflection occurs, $v$ is the ultrasound velocity in the medium, $t$ is the time which takes for the ultrasound to return to the transducer.

When the ultrasound wave travels from one medium to another, a portion of the signal will reflect and the rest of the signal will be transmitted into the second medium. Reflection coefficient is used to estimate the portion of ultrasound reflected when the sound waves travel from one medium to another. The reflection coefficient depends on the acoustic impedance of the two medium materials [62]. For example, the higher the difference between the two mediums in acoustic impedance, the higher the reflection coefficient. Equation 2.4 shows the acoustic impedance of a particular medium whereas Equation 2.5 shows the estimation of the reflection ratio between two mediums [62].

$$
Z=\rho \cdot v
$$

where $Z$ represents the acoustic impedance, $\rho$ is the density of the material and $v$ is the ultrasound velocity in the medium.

$$
R=\frac{\left(Z_{1}-Z_{2}\right)^{2}}{\left(Z_{1}+Z_{2}\right)^{2}}
$$

where $R$ is the reflection coefficient of the ultrasound at the boundaries of the two 
mediums, $Z_{1}$ and $Z_{2}$ are the acoustic impedance of the materials on the second material.

In addition, the strength of the echo signal captured in RF signal also depends on the amount of ultrasound energy reflected back to the transducer. Due to the high impedance of the bone, reflection ratio between the bone and muscle is high. Most of the echo signal is reflected from the bone. Figure 2.7 shows a schematic of multi-layer tissue thickness measurements by a single transducer with pulse echo technique and the corresponding ultrasonic RF signal. Air has an extremely low acoustic impedance

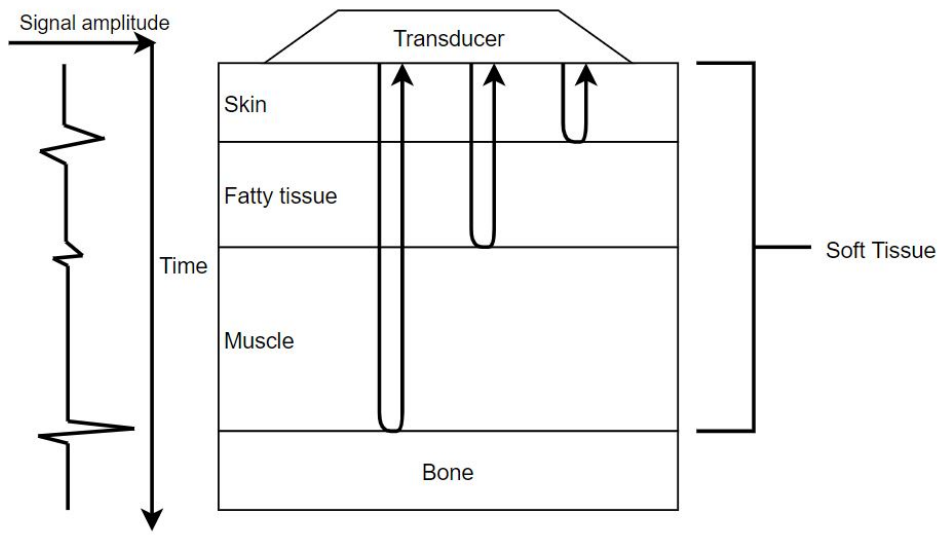

Figure 2.7: Diagram of a pulse-echo ultrasonic measurement and the corresponding RF signal.

when compared with other body materials. If the transducer is connected with the surface of the tissue directly, a layer of air might separate them. Most of the signal will reflect back to the transducer without transmitting into the tissue. In order to remove the air between the transducer and the surface of the object, an ultrasonic gel is used [62]. Due to the fact that the acoustic impedance of the coupling gel and human tissues are similar, most of the ultrasound signals are able to transmit into the tissue. Throughout the study, the ultrasonic gel is placed between the transducer and the investigated object. 


\subsubsection{Ultrasound Attenuation}

The travelling ultrasonic pulse loses strength gradually due to scattering and absorption. The loss of ultrasonic pulse strength is called attenuation. The amplitude of the ultrasonic signal after it propagates through the medium can be calculated by the Equation 2.6 [67].

$$
A(x)=A o \cdot e^{-\mu \cdot d}
$$

where $A o$ is the original amplitude of the ultrasonic signal, $\mu$ is the attenuation coefficient and $d$ is the distance traveled by the ultrasound pulse. The degree of attenuation depends on the frequency of the ultrasonic beam, the characteristics of the medium, and the travelling depth. While increasing the ultrasonic frequency, the spatial resolution will also be improved. However, the penetration depth will be reduced [67]. Attenuation coefficient $(\mu)$ of the material is used to describe the attenuation of the ultrasonic signal in that particular material. The unit of attenuation coefficient in biological tissue is $\mathrm{dB} /(\mathrm{cm} \cdot \mathrm{MHz})$. The amplitude of the ultrasonic attenuation is also exponentially increased due to the travelling distance. Thus, the further the ultrasonic pulse travels, the higher the attenuation.

\subsubsection{Scattering}

Scattering occurs when the ultrasonic pulse encounters the boundaries of particles with distinguished acoustic impedance from the surrounding medium, and the dimension of the particles are smaller than, or comparable to, the wavelength of the ultrasound waveform. When the aforementioned conditions are met, normal reflection law do not apply to the ultrasonic waveform and the ultrasonic wave scatters in multiple directions [68]. As the direction of the scattering effect is random, some 
ultrasound waves may bounce within the medium and return to the transducer. As a result, the travelling distance of the ultrasonic pulse or the time delay before reaching to the transducer is not constant. It is challenging to filter the scattering noise since the frequency shared between the ultrasonic signal and the scattered ultrasonic noise are identical [67]. In Chapter 4, other DSP techniques are applied to suppress the scattering noise from the RF signal.

\subsubsection{Hand-Held Conventional Ultrasound Probe}

The ultrasonic transducer is made from piezoelectric materials which converts electrical pulses into ultrasonic pulses and vise versa. Amplitude ultrasonic mode (A-mode), which can be obtained from a single transducer, is able to capture the thickness between tissue boundaries as well as the dynamic motion [17,67]. Multiple transducers are aligned and operate simultaneously to obtain an ultrasound image. In other words, the ultrasonic brightness mode image (B-mode) is constructed by multiple A-mode signals.

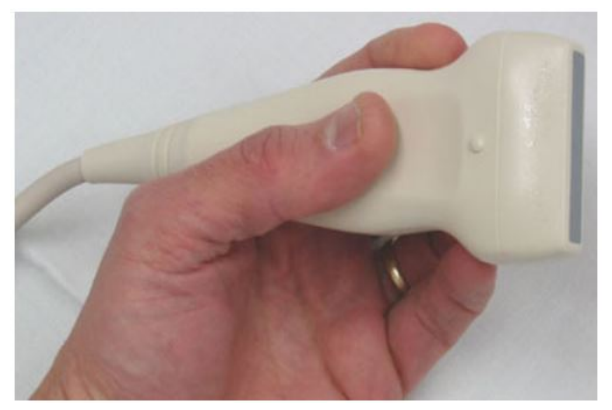

Figure 2.8: A hand-held conventional ultrasonic probe. Image is reproduced from "Medical Ultrasound linear array Probe/scan head/transducer" by D. W. Rickey and the image is licensed under Creative Commons Attribution-Share Alike 2.5 Generic license. Image is free access online at https://commons.wikimedia.org/wiki/File:UltrasoundProbe2006a.jpg [65].

Figure 2.8 shows an image of the hand-held conventional linear array ultrasonic probe [69]. A previous study has mentioned the measurement errors caused by the 
hand-held conventional probe [10]. The hand-held conventional ultrasonic probe is bulky in shape and heavy, which may lead to the inconsistent movement of the probe with respect to the skin surface. The weight and pressure applied by the conventional probe may restrict the tissue motion during signal acquisition. Hence, such a probe may not be suitable for long-term continuous tissue motion monitoring [10]. 


\section{Chapter 3}

\section{Wearable Ultrasonic Sensor and DSP Techniques}

In this chapter, the wearable ultrasonic sensor (WUS) is introduced for muscle tissue monitoring. The specification of the WUS and ultrasonic measurement system configuration are provided. The raw ultrasound RF signal obtained from the ultrasonic system is pre-processed to improve the signal-to-noise ratio (SNR) of the desired sig-

nal. After that, the thickness of the tissues can be estimated from the ultrasound RF signal. Three different techniques of estimating displacement from A-mode measurements are peak detection, cross-correlation, and quadrature demodulation. This chapter ends with a brief introduction of each of the aforementioned measurement techniques.

\subsection{Wearable Ultrasound Sensor}

One of the goals in this thesis is to provide a reliable method for tissue monitoring during muscle contraction by using an ultrasound measurement in pulse-echo mode. 
There are some limitations in the current ultrasonic monitoring methods. First of all, the skin surface has a natural curvature that is unique from person to person. With the commercial handheld ultrasound probe, it is challenging for long term monitoring. In order to prevent undesired motion artifacts caused by unsteady hand motion, a flexible and wearable sensor is more feasible in long term monitoring. Better contact between the skin and the ultrasound sensor also increases the return ultrasonic signal stability. To prevent the challenges in muscle monitoring with the conventional probe, a flexible and wearable ultrasonic sensor (WUS) is developed based on the design by I. Almohimeed, et al. [70,71]. The WUS, used in this study are constructed by the author, are specific developed for tissue and muscle monitoring applications. A review of the WUS and the acquisition system design by I. Almohimeed, et al. will be discussed in this section.

The WUS is constructed from a $110 \mu \mathrm{m}$ polyvinylidene fluoride (PVDF) polymer piezoelectric film (Model: 2-1004346-0, Measurement Specialities Inc., Hampton, VA, USA) [72]. Compared to lead ziroconate titanate (PZT), which is the most commonly used piezoelectric material in constructing the medical use ultrasonic probe, the PVDF has a closer acoustic impedance that matches to human tissues than the PZT. The acoustic impedance of human tissues, PVDF and PZT are $1.63 \times 10^{6} \mathrm{~kg} /\left(\mathrm{m}^{2} \mathrm{~s}\right)$ [73], $3.43 \times 10^{6} \mathrm{~kg} /\left(\mathrm{m}^{2} \mathrm{~s}\right)$ and $32 \times 10^{6} \mathrm{~kg} /\left(\mathrm{m}^{2} \mathrm{~s}\right)$ [74], respectively. Hence, the reflection ratio of PZT-human tissue and PVDF-human tissue are 0.82 and 0.13 , respectively. Since the reflection ratio between PVDF and human tissue is low, most of the ultrasonic signal can be transmitted into human tissues from the transducer without acoustic matching layer, which is required for a PZT ultrasonic transducer. Table 3.1 shows the acoustic impedance in other commonly used piezoelectric materials in constructing the ultrasonic probe [75]. The flexibility of the PVDF polymer provides a better fit to the body surface. Thus, the motion of the tissues is not restricted by 
the WUS. Additionally, it is barely noticeable when wearing the WUS due to its light weight $(\approx 0.2 \mathrm{~g})$. With the WUS, it is possible to monitor tissue motion while doing physical activities such as sports and fitness [70,71].

Table 3.1: Acoustical properties of several piezoelectric materials [60-62].

\begin{tabular}{|c|c|c|c|}
\hline Material & $\begin{array}{c}\text { Density } \\
\left(\mathrm{kg} / \mathrm{m}^{3}\right)\end{array}$ & $\begin{array}{c}\text { Propagation Velocity } \\
(\mathrm{m} / \mathrm{s})\end{array}$ & $\begin{array}{c}\text { Acoustic Impedance } \\
\left(\mathrm{kg} /\left(\mathrm{m}^{2} \mathrm{~s}\right)\right)\end{array}$ \\
\hline Quartz $\left(\mathrm{SiO}_{2}\right)$ & 2650 & 5760 & $15.3 \times 10^{6}$ \\
\hline $\begin{array}{c}\text { Zirconate piezoceramic } \\
(\text { PZT-5A) }\end{array}$ & 7750 & 3880 & $30 \times 10^{6}$ \\
\hline $\begin{array}{c}\text { Zirconate piezoceramic } \\
(\text { PbNb 2 0 6) }\end{array}$ & 5800 & 2800 & $16 \times 10^{6}$ \\
\hline Piezopolymer (PVDF) & 1780 & 2260 & $4.6 \times 10^{6}$ \\
\hline
\end{tabular}

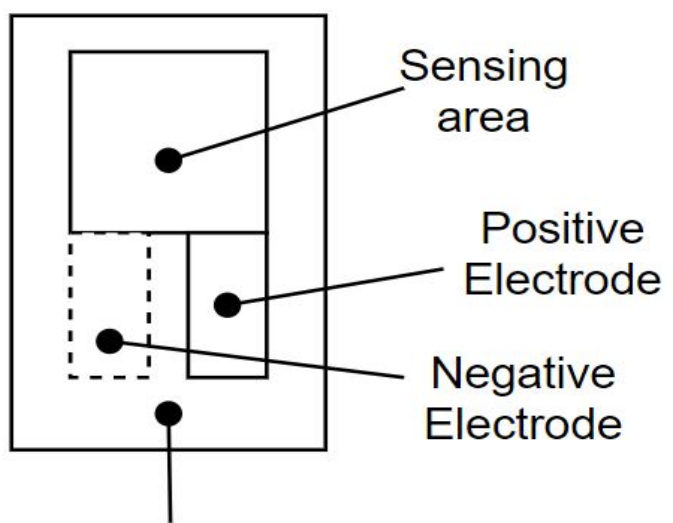

PVDF

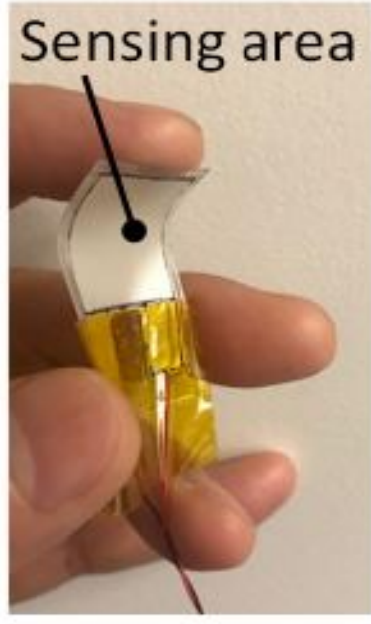

(b)

Figure 3.1: Schematic representation of the WUS (a) and a photograph of the WUS (b).

Figure 3.1 (a) shows the schematics and (b) a photo of the constructed WUS. The WUS is constructed with the PVDF film with a sensing area of $2 \mathrm{~cm} \mathrm{X} 2 \mathrm{~cm}$, and the electrode is a silver ink coating. A copper wire is connected to each side of the electrodes. The PVDF is sensitive to heat and the melting point is from 160 
to $300{ }^{\circ} \mathrm{C}$ [72]. Instead of regular heat soldering to connect the copper wires with the PVDF, silver epoxy (8331 Silver Conductive Epoxy Adhesive, Canada) is used. Since silver epoxy has a low resistivity to electricity $(0.007 \Omega / \mathrm{cm})$, it has a minimal impact to alternate the flow of the current [76]. The silver epoxy is applied to the non-sensing area of the PVDF electrode, where it will not affect the sensing area of the PVDF. The positive and negative electrode wire connection areas are protected by a protection film to prevent the contact between the subjects with the copper wires as shown in Figure (b). With the sensing area of $2 \mathrm{~cm} \mathrm{X} 2 \mathrm{~cm}$ and $110 \mu \mathrm{m}$ thickness, the estimated operating frequency is $6.9 \mathrm{MHz}$. The chosen sensing area size is due to the consideration of the near-field and far-field effects. Figure 3.2 shows a schematic of the sensor performances such as signal strength, and near-field and far-field effects [77]. Within the near-field region, the ultrasound pulses maintain a relatively constant ultrasound beam area that is ideal for the sensing area $[77,78]$. The near-field zone of a transducer with a square sensing area is calculated by Equation 3.1 [79] and the result of varying sizes of the sensing area are shown in Table 3.2. This sensor is typically designed for monitoring forearm and shoulder tissues since the total thicknesses of these tissues are smaller than $30.2 \mathrm{~cm}$ in average male subjects $[80,81]$.

Table 3.2: The near-field distance corresponding to various sizes of the ultrasonic transducer.

\begin{tabular}{|c|c|c|c|c|}
\hline Sensing Area & $1 \mathrm{cmX} 1 \mathrm{~cm}$ & $1.5 \mathrm{cmX} 1.5 \mathrm{~cm}$ & $2 \mathrm{cmX} 2 \mathrm{~cm}$ & $2.5 \mathrm{cmX} 2.5 \mathrm{~cm}$ \\
\hline Near-Field Distance & $15.1 \mathrm{~cm}$ & $22.7 \mathrm{~cm}$ & $30.2 \mathrm{~cm}$ & $37.8 \mathrm{~cm}$ \\
\hline
\end{tabular}

$$
N=\frac{1.35 f_{c} D^{2}}{4 v_{h}}
$$




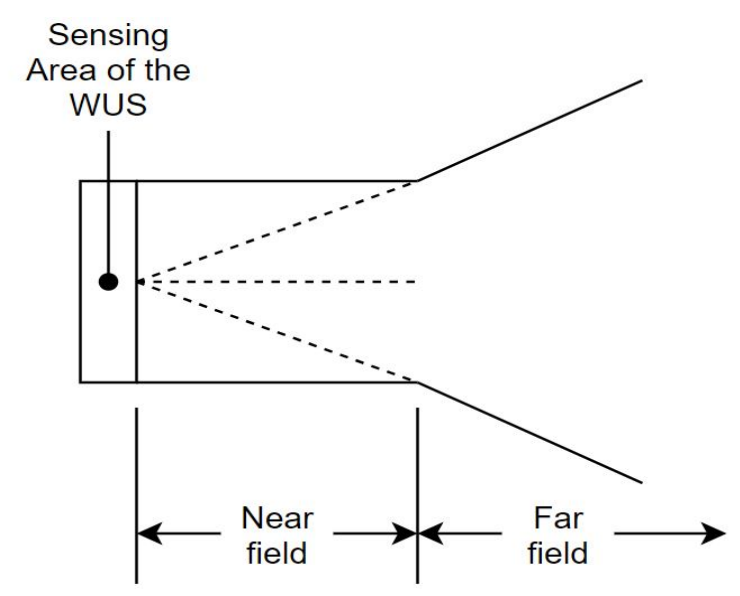

Figure 3.2: The near-field and far-field demonstrations. The image is reproduced by the author of this thesis based on the information discussed in "Diagnostic Ultrasound: Physics and Equipment" by P. Hoskins, et al. [7r7].

Where $\mathrm{N}$ is the near-field distance; $\mathrm{f}_{c}$ is the center frequency of the transmitting ultrasound; $D$ is the length of the ultrasound transmitter in $\mathrm{cm}$; and $v_{h}$ is the average ultrasound velocity in human soft tissue, which is assumed to be $(1540 \mathrm{~m} / \mathrm{s})$ [67]. In our previous study, the WUS demonstrated the feasibility in muscle thickness monitoring of the index finger and forearm in pulse-echo mode [70]. Another study by our group, a body wearable stiffness sensor has been developed for continuous monitoring of the mechanical properties of plantar soft tissues at the heel region in a shoe [82]. Tissues stiffness can be measured by the quasi-static elasticity method, which was defined as the simultaneous measure of the pressure and tissue displacement.

Figure 3.3 shows the schematic diagram of the measurement configuration in thickness estimation by the WUS. The WUS is driven by an ultrasonic pulser-receiver (Model: DPR300, JSR Ultrasonics, Pittsford, NY, USA). The received radio frequency $(\mathrm{RF})$ ultrasonic signals is digitized at $125 \mathrm{MHz}$ sampling rate by a 14 bits data acquisition system (Model: ATS 9440, Alazartech, Montreal, QC, Canada). The frame rate is 1000 frames per second, controlled by a function generator. Since the 


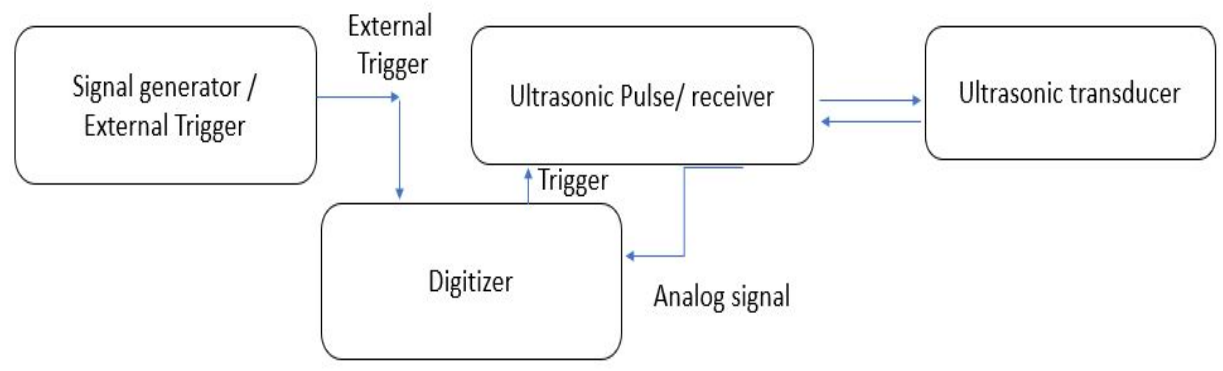

Figure 3.3: Ultrasound Measurement System

average minimum frequency to evoke tetanus in fast twitching muscle is lower than 50 $\mathrm{Hz}$, the chosen frame rate is capable to capture the entire motion of the contraction [47]. In this study, the slow time is referred to the measurement time (associated with the frame rate) and the fast time within a single frame corresponds to the propagation time in the tissue (associated with the tissue depth). The acquisition window size in the fast time must be longer than the TOF of the ultrasound echo within the medium; otherwise the ultrasound measurement will not be able to capture the reflected ultrasound echo of interest. In total, 14400 samples are acquired in each frame, and the sampling interval is $8 \mathrm{~ns}(125 \mathrm{MHz})$ in the WUS measurement system. Hence, the fast time in each frame is equal to $115.2 \mu \mathrm{s}$ (14400 samples multiple with $8 \mathrm{~ns}$ ), which is equivalent to $\approx 88.7 \mathrm{~mm}$ depth in the average human soft tissue in ultrasonic pulse echo mode, as demonstrated in Equation 3.2. The pulser-receiver device contains an internal band pass filter in the frequency range from $1 \mathrm{MHz}$ to $12.5 \mathrm{MHz}$. Since all of the acquisition devices are connected to the same external trigger source, all devices are running under the same clock cycle. Therefore, the maximum measurement error in time is $8 \mathrm{~ns}$ due to the sampling frequency of the acquisition system being $125 \mathrm{MHz}$. The RF signal obtained from the acquisition system is then processed in MATLAB for pro-processing and analysis for tissue thickness measurement. 


$$
d_{t}=\frac{v \cdot t}{2}
$$

where $d_{t}$ is the distance ultrasound echo travelled, $v$ is the ultrasound velocity in the medium and $t$ is the time taken for the ultrasound to travel within the medium.

\subsection{Pre-processing Technique}

As the ultrasonic signal travels through the tissue, the amplitude of the signal is attenuated due to scattering, absorption, reflection and diffraction. Noise, such as electrical noise, may also induce change in echo signal amplitude and echo signal shape. SNR is commonly calculated by dividing the peak to peak amplitude of the echo of interest with the root mean square of the noise. Measurement errors might occur due to the poor SNR. Moving averaging is one of the preprocessing techniques applied to the acquired raw RF ultrasound signals in order to suppress the noises and increase the SNR. Figure 3.4 shows the visual operation of the moving averaging in the raw RF signals. In this thesis, we refer the frame rate as slow time and the time within a single frame is called the fast time. The RF signal stored in the current frame is averaged with the surrounding frames in slow time. For example, 5 frames moving averaging is corresponding to the moving averaging of the current frame, the two previous frames and the following two frames. The frame rate of $1000 \mathrm{~Hz}$ is selected for the acquisition system. However, the frame rate is reduced due to moving averaging. For an example, the 5 frames moving averaging has reduced the frame rate to $200 \mathrm{~Hz}$.

In general, the greater the number of frames averaging the higher the SNR. However, moving average might lead to the smoothing of the tissue motion measurement. In order to capture more detail of the muscle motion, the signal acquisition frame 


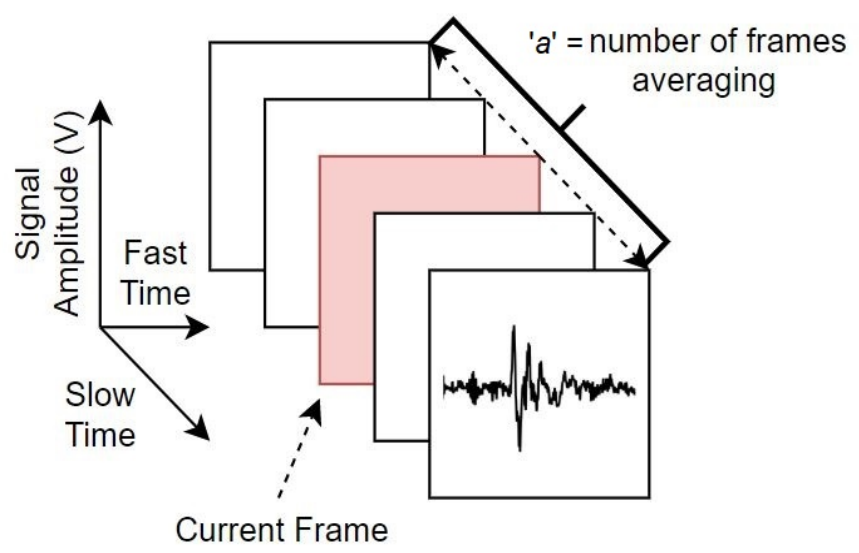

Figure 3.4: The operation of the moving averaging on the ultrasonic RF signals.

rate must be high enough. Assuming the muscle displacement evoked by EMS can be represented with a sinusoidal function. By sampling twice of the frequency of the sinusoidal function, all the Fourier components can be capture and recovered based on the Nyquist-Shannon sampling theorem $[73,83]$. Therefore, the frame rate after moving averaging has to be at least twice the EMS evoked frequency during muscle monitoring. The effect of moving averaging on the accuracy of the thickness measurements are discussed in Chapter 5.1.

\subsection{Thickness Changes Estimation Methods}

The RF signal contains the information of the echoes reflected from the boundaries of the materials. Three muscle thickness changes estimation methods are investigated from the recorded RF signal, and the relative resolution for each method will be discussed in the following paragraphs. 


\subsubsection{Peak Detection}

The peak detection $(\mathrm{P} / \mathrm{D})$ method is a common thickness estimation method and is used to locate the highest amplitude of the echo within a RF signal. When the sound pulse travels from one medium to another, some sound pulses reflect with respect to the reflection ratio. If the second medium is a relative high-density (high acoustic impedance) material such as bone or glass, most of the sound energy will be reflected back to the receiver. Therefore, the received echo has a relative high amplitude in the RF signal. The entire tissue thickness between the WUS and the bone including skeletal muscle can be calculated from the echo TOF in the RF signal, which is the sound reflection from the bone. The main advantage of $\mathrm{P} / \mathrm{D}$ method is that it does not require high computational power to operate since the signal processing is performed in the time domain of the RF signal. From the time delay of the echo and ultrasound velocity of medium, the distance of the sound travel can be calculated by Equation 3.2. Some disadvantages associated with this method may affect the accuracy of the measurement. Since the P/D is very sensitive to noise, any disruption to the shape of the echo may alter the measurement result. Occasionally, the neighbor peak may have a higher amplitude than the target peak due to poor signal strength or scattering noise.

\subsubsection{Cross Correlation}

The cross-correlation $(\mathrm{C} / \mathrm{C})$ method is used to find the best correlation between two consecutive RF signals. It can also discover the time delay between the two RF signals. The $\mathrm{C} / \mathrm{C}$ method is more immune to noise than the peak detection since the $\mathrm{C} / \mathrm{C}$ is using the entire $\mathrm{RF}$ signal within a chosen time window to obtain the time delay of the echoes. By finding the time delay where the amplitude of the $\mathrm{C} / \mathrm{C}$ 
coefficient is highest, we can obtain the thickness changes of the material between the two consecutive frames.

$$
\begin{gathered}
G=E \otimes H \\
G(i)=\sum_{u=-k}^{k} E(u) H(i+u)
\end{gathered}
$$

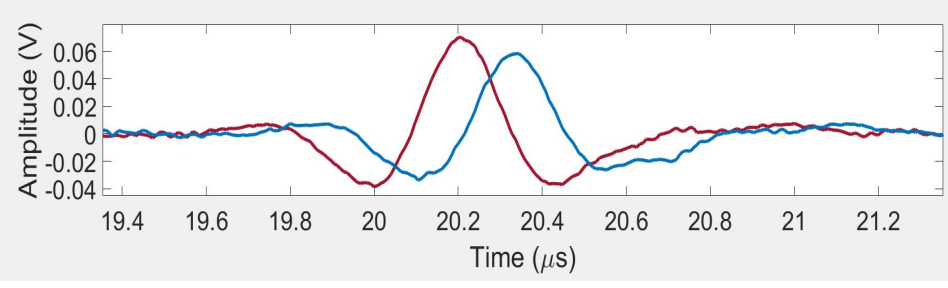

(a)

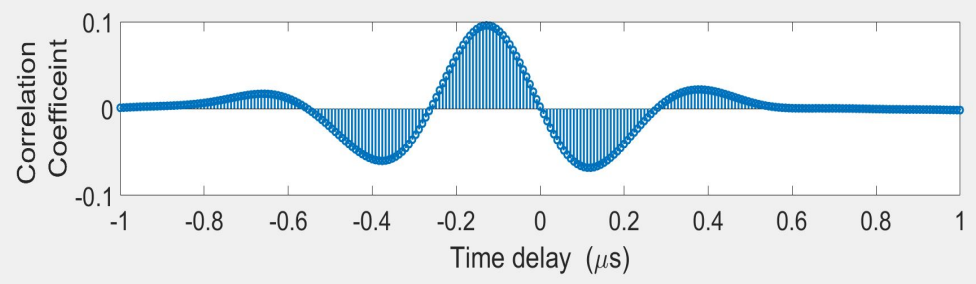

(b)

Figure 3.5: (a) The reference ultrasonic echo in red and the shifted ultrasonic echo in blue and (b) the correlation coefficient of the two signals.

Figure 3.5 (a), shows the zoomed-in reference echo signal in red $(E)$ and the consecutive echo signal in blue $(H)$. Equation 3.3 shows the cross-correlation formula in which $G$ is the correlation coefficient. Where $k$ is the total number of samples within the time interval, $u$ is the lag and $i$ is the sample index. Figure 3.5 (b) shows the correlation coefficient of the two signals. The highest amplitude of correlation coefficient at 0.1 is the best coherence between the two signals from the example RF signals. The time delay at the peak is approximately $-0.18 \mu$ s. Both peak detection and cross-correlation are calculated in the time domain in which the target boundaries 
displacement can be calculated by Equation 3.2. The $\mathrm{C} / \mathrm{C}$ method is able to detect the relative thickness changes by using the first RF signal as a reference. However, it is unable to measure the initial thickness of the medium or tissues. Other methods such as peak detection are needed to provide the initial thickness information of the medium.

\subsubsection{Quadrature Demodulation}

During muscle contraction, the ultrasound travelling distance between the WUS and the tissue boundary changes. This will induce a time shift of the target echo between the consecutive frames. This time shift causes a change in phase between the ultrasound signal and a reference signal in which it is associated with the tissue thickness changes [10]. By using quadrature demodulation $(\mathrm{Q} / \mathrm{D})$, the instantaneous phase shift can be obtained from the acquired RF signal. Additionally, the Q/D is one of the most commonly used method for signal analysis in medical ultrasounds $[84,85]$. It is often used for ultrasound diagnostic techniques such as ultrasound elasticity and acoustic radiation force imaging $[86,87]$. The details of the quadrature demodulation technique is given in the Appendix A.1.

After acquiring the phase information, the thickness changes between two consecutive frames can be calculated by

$$
\Delta d(n)=\frac{v \cdot \Delta \theta(n)}{4 \pi \cdot f_{c}}
$$

where $\Delta d(n)$ is the displacement estimation predicted according to the phase shift $\Delta \theta(n)$ obtained from quadrature demodulation, $v$ is the ultrasound velocity of the medium and $f_{c}$ is the center frequency of the ultrasound probe. This conversion from the phase information to displacement is called the phase tracking method. Consid- 
ering the minimal phase changes obtained from Q/D of 0.1 radians, the resolution of the phase tracking method is $1.8 \mu \mathrm{m}$. The accumulated displacement $\mathrm{d}(\mathrm{n})$ is calculated by the previous accumulated displacement $\mathrm{d}(\mathrm{n}-1)$ and the $\Delta d(n)$ between the two consecutive frames in Equation 3.4.

$$
d(n+1)=d(n)+\Delta d(n)
$$

There is a backdrop in the phase sensitive method, in which the displacement estimation is based on the phase change between two consecutive frames. Thus, the phase shift cannot be greater than $2 \pi$ radians. Any motion greater than $2 \pi$ radians cannot be recognized as the reference signal is a continuous sinusoidal function. To overcome this limitation, the frame rate of the ultrasonic acquisition system must be faster than the tissue motion. With this WUS measurement system, the maximum speed of the thickness changes $(\mathrm{vm})$ is calculated by the following

$$
v m=\frac{\Delta d \max }{t m}
$$

where $\Delta d \max$ is the maximum displacement by phase sensitive method and $\mathrm{tm}$ is the sampling time between two consecutive frames in the measurement system. As the maximum phase shift is $2 \pi$ radians the $\Delta d \max$ is equal to $0.1116 \mathrm{~mm}$. The frame rate chosen in the ultrasonic measurement system is $1000 \mathrm{~Hz}$, which is equivalent to $1 \mathrm{~ms}$. Therefore, the maximum measurement speed in phase tracking method is $111.6 \mathrm{~mm} / \mathrm{s}$. Since the isometric contraction velocity in most of the forearm muscle is around $3 \mathrm{~mm} / \mathrm{s}$ to $20 \mathrm{~mm} / \mathrm{s}$ [88]. Therefore, WUS measurement system using the quadrature demodulation and the phase tracking method is capable of estimating the 
thickness changes of the forearm muscle due to isometric contraction. 


\section{Chapter 4}

\section{Accuracy Evaluation on Thickness}

\section{Measurement Using Gelatin-based}

\section{Phantom}

This chapter discusses the accuracy of tissue thickness measurements using the WUS. In order to evaluate this, a LDS is used as a gold standard. The controlled experiments are conducted using a phantom that mimics soft tissue properties. The phantom composition and construction process are presented in this chapter. The phantom thickness is changed by a mechanical force which pushes the phantom on the side and deforms its shape. During the experiment, the changes in thickness are measured using the WUS and a LDS. Two controlled experiments have been conducted for accuracy evaluation. The first experiment evaluates and compares the accuracy of three different thickness signal processing techniques mentioned in Chapter 3, including peak detection, cross-correlation and phase tracking methods. As the thickness estimation method has the highest resolution and accuracy, it will be used in the following experiments and throughout the thesis. The second experiment is a 
mechanical simulation to simulate a muscle contraction evoked by electrical stimulation. The purpose of the second experiment is to investigate the repeatability and accuracy of the WUS measurement system in measuring thickness changes.

\subsection{Soft Tissue Mimicking Phantom}

\subsubsection{Review of Gelatin-Based Phantom}

Evaluation of measurement accuracy is performed using a phantom that mimics the desired mechanical properties of soft tissue. The main property of the tissue mimicking phantom required in this study is that the elastic phantom is able to recover to its original shape after an external force is released from it. Another phantom property is the ultrasound velocity. The ultrasound wave propagates through various biological tissues with varying velocities [66]. Furthermore, the tissue ultrasound velocities are different from person to person and tissue to tissue. Therefore, it is difficult to account for all the different ultrasound velocities in the tissue thickness measurement. To simplify this problem, it is common to use a constant ultrasound velocity in medical ultrasound imaging to measure the tissue thickness changes [67]. In this study, a constant ultrasound velocity is used to estimate the thickness of the phantom.

A gelatin-based phantom is commonly used in mimicking the soft biological tissues due to its similarity in elastic and acoustic properties [89-91]. Therefore, gelatin-based phantoms are often used for developing and testing ultrasound strain imaging techniques and ultrasound-guided therapy training exercises [92-94]. Gelatin is a protein derived from the collagen of animal skin and bones. With different concentrations of the gelatin in the phantom, its stiffness and the ultrasound velocity can be controlled 
[90,91]. Table 4.1 shows the acoustic properties of the gelatin-based material and of some of the biological tissues $[65,90,91,95]$.

Table 4.1: Acoustic properties of the gelatin-based materials and biological tissues $[65,89,90,94]$.

\begin{tabular}{|c|c|c|c|c|c|}
\hline Material & $\begin{array}{l}\text { Sound } \\
\text { Velocity } \\
(\mathrm{m} / \mathrm{s})\end{array}$ & $\begin{array}{l}\text { Density } \\
\left(\mathrm{kg} / m^{3}\right)\end{array}$ & $\begin{array}{c}\text { Attenuation } \\
\text { Coefficient } \\
(\mathrm{dB} / \mathrm{cm} \cdot \mathrm{MHz})\end{array}$ & $\begin{array}{c}\text { Acoustic } \\
\text { Impedance } \\
(\text { MRayl) }\end{array}$ & Source \\
\hline $\begin{array}{c}\text { Gelatin-based } \\
\text { Phantom }\end{array}$ & $1500-1650$ & 1050 & $0.12-1.5$ & $1.60-1.73$ & $\begin{array}{c}\text { Cook2011 } \\
{[91]}\end{array}$ \\
\hline Fat & 1478 & 950 & 0.48 & 1.40 & $\begin{array}{c}\text { Culjat2010 } \\
{[90]}\end{array}$ \\
\hline Muscle & 1547 & 1050 & 1.09 & 1.62 & $\begin{array}{c}\text { Culjat2010 } \\
{[90]}\end{array}$ \\
\hline Liver & 1595 & 1060 & 0.50 & $1.63-1.75$ & $\begin{array}{l}\text { ICRU1998 } \\
{[95]}\end{array}$ \\
\hline $\begin{array}{c}\text { Soft Tissue } \\
\text { (Average) }\end{array}$ & 1540 & 1043 & $0.54-1.00$ & $1.00-1.63$ & $\begin{array}{c}\text { Wolbarst1993 } \\
{[65]}\end{array}$ \\
\hline
\end{tabular}

Culijat, et al. investigated the relationship between the acoustic properties and the gelatin concentration [90]. The gelatin concentration is calculated by the weight of the gelatin powder divided by the weight of the water. For example, 10 wt\% gelatin concentration is equivalent to $10 \mathrm{~g}$ of gelatin powder in every $100 \mathrm{~g}$ of water. It was found that a $12 \mathrm{wt} \%$ of gelatin concentration can obtain an ultrasound speed close to $1540 \mathrm{~m} / \mathrm{s}$ [90]. The attenuation coefficient of $12 \mathrm{wt} \%$ gelatin in $6.9 \mathrm{MHz}$ is around $0.8 \mathrm{~dB} / \mathrm{cm}[90]$. Sakai, et al. investigated the increase in elasticity by changing the concentration of gelatin in water [96]. The elasticity for $12 \mathrm{wt} \%$ gelatin measured in Young's modulus is in the range of 20 to $30 \mathrm{kPa}$ [96]. The elasticity of human muscles and soft tissues such as gastrocnemius medialis and gastrocnemius lateralis have an elasticity of 22.5 to $24 \mathrm{kPa}$ [97]. Therefore, a phantom with $12 \mathrm{wt} \%$ gelatin concentration is capable of mimicking the acoustic and elasticity properties of 
biological soft tissues.

\subsubsection{Phantom Construction}

Two gelatin-based phantoms are made with 12 wt\% gelatin concentration. The 12 wt\% gelatin solution is made from dissolving $12 \mathrm{~g}$ of gelatin powder in every $100 \mathrm{ml}$ of distilled water. The distilled water is heated to speed up the process in dissolving the gelatin powder. The shape of the gelatin-based phantom is determined by the shape of the mold during solidification. A mold with a flat top and bottom surfaces is chosen to allow full contact of the WUS with the fabricated phantom. Graphite particles are added into the phantom to create some scattering effect, which also increases the acoustic attenuation in the phantom [10,91]. The $12 \mathrm{wt} \%$ gelatin solution is placed in the refrigerator to speed up the process of solidification which prevents the graphite powder from settling at the bottom of the mold [10]. Table 4.2 includes the amount and composition of the materials in constructing the soft tissue mimicking phantom.

The step-by-step procedure in constructing the phantom is given as follows:

1. Heat the distilled water in a glass container.

2. Slowly pour the gelatin powder into the beaker to prepare a $12 \mathrm{wt} \%$ concentration gelatin solution. Stir the solution to ensure the powder is fully dissolved within the solution while maintaining the temperature.

3. Add graphite powder into the solution.

4. Pour the $12 \mathrm{wt} \%$ gelatin solution into a mold. Ensure there is no undissolved gelatin powder in the solution.

5. Remove any air bubbles within the solution by using a glass rod or laboratory spatula.

6. Put the solution and the container into a refrigerator set to 0 to $5^{\circ} \mathrm{C}$ for approximately 24 hours. 
Table 4.2: The composition and materials used in the gelatin-based phantom.

\begin{tabular}{|c|c|c|c|}
\hline Material & Distilled Water & Gelatin Powder & Graphite Powder \\
\hline Composition Percentage & $87.5 \%$ & $12 \%$ & $0.5 \%$ \\
\hline Example of Composition & $500 \mathrm{~g}$ & $60 \mathrm{~g}$ & $2.5 \mathrm{~g}$ \\
\hline
\end{tabular}

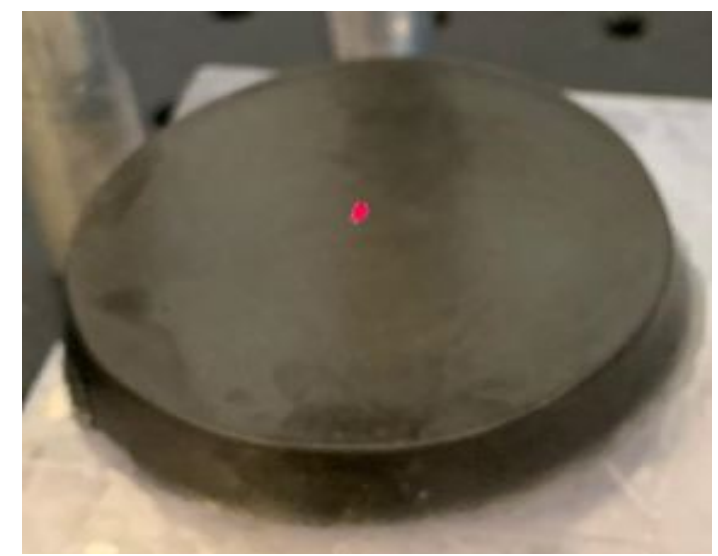

(a)

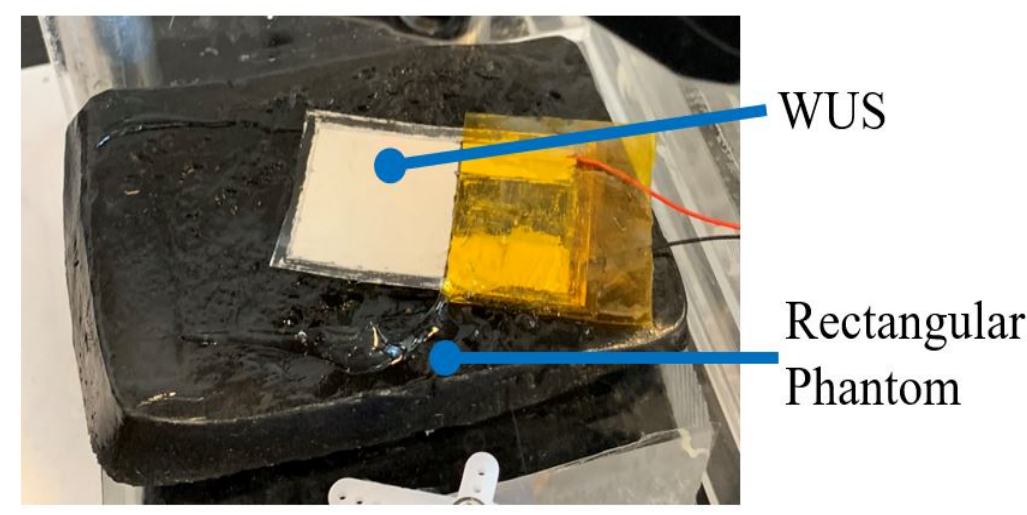

(b)

Figure 4.1: Photographs of the cylinder phantom (a) and the rectangular phantom (b).

For the storage of the constructed phantom, it is placed within a shield container and stored inside the refrigerator to prevent the gelatin from drying and inhibit mold creation. The dried phantom may lose its elasticity and may fracture when experiencing an external force. Since the ultrasound speed of the phantom changes 
according to the temperature, the phantom is kept at room temperature $\left(21^{\circ} \mathrm{C}\right)$ for approximately 1 hour before performing the ultrasound experiment.

Two phantoms are constructed using the composition listed in Table 4.1. The first phantom is constructed by a cylinder mold with a diameter of $40 \mathrm{~mm}$ and a height of $10 \mathrm{~mm}$. Figure 4.1 (a) shows an image of the cylinder shaped phantom. The shape and size of the cylinder shaped phantom allow it to be easily squeezed by hand to simulate voluntary muscle isometric contraction in section 4.2. The second phantom is constructed by a rectangular mold with $40 \mathrm{~mm}$ width $\mathrm{X} 40 \mathrm{~mm}$ length and $20 \mathrm{~mm}$ height. The shape of this phantom is chosen to allow the application of uniform mechanical force. The rectangular shaped phantom is used in section 4.3.

The purpose of the phantom is to provide an elastic medium where the thickness is changeable. The exact match of the elasticity of the phantom to soft tissue is not the focus of the study. Since human soft tissues are complex and ultrasound travels through different soft tissue layers in varying velocities, it is common to use an average ultrasound velocity in medical ultrasound imaging $[67,98]$. The ultrasound velocity is measured at the beginning of the phantom experiment for thickness estimation. Hence, the adjustment of the ultrasound velocity of the phantom is not the main concern in the development of the phantom. Other soft tissue behaviors are also out of the scope of this thesis.

\subsubsection{Phantom Ultrasound Velocity and Acoustic Attenua- tion}

In order to estimate the thickness changes of the phantom by the WUS measurement system, the ultrasound velocity of the phantom is needed. By rearranging the timeof-flight (TOF) equation in pulse-echo mode (Equation 3.2), the ultrasound velocity 
$\left(v_{p}\right)$ of the phantom can be estimated by equation 4.1 . The time delay $(\Delta \mathrm{t})$ is the duration of the ultrasound echo returned to the ultrasound transducer. The initial thickness of the phantom $\left(d_{o}\right)$ is measured by the LDS. Since the ultrasound echo propagates through the phantom twice in pulse echo mode, the $d_{o}$ is multiplied by 2 .

$$
v_{p}=\frac{d_{o} \cdot 2}{\Delta t}
$$

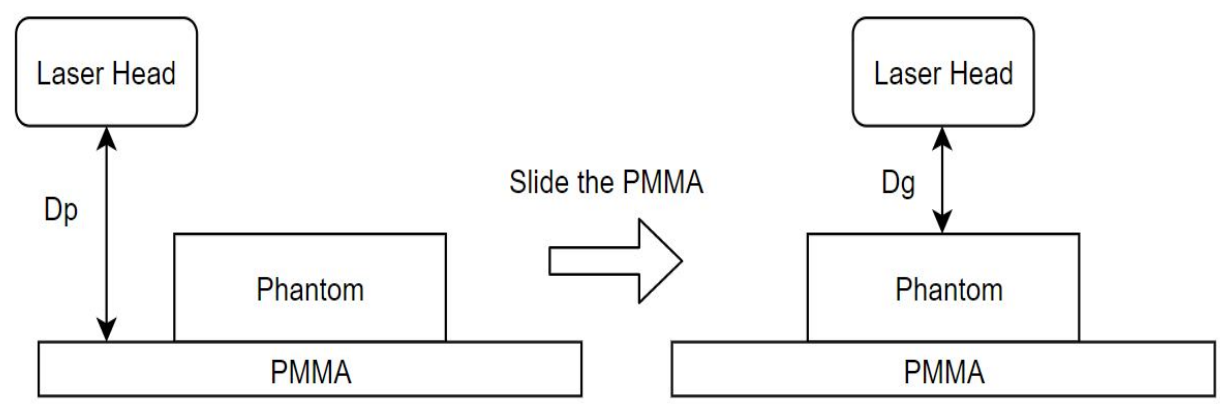

Figure 4.2: Measurement configuration and procedure of phantom thickness estimation using $L D S$.

The experimental configuration and procedures for measuring the total thickness of the opaque phantom using the LDS (Model: LK-H055K, Keyence, Japan) is as shown in Figure 4.2. The phantom is placed on top of the polymethylmethacrylate (PMMA) plate, and the distance (Dp) between the LDS head and the top of the PMMA plate is measured by the LDS. Then, the PMMA plate with the phantom is moved carefully, and the distance (Dg) between the LDS head and the top of the phantom is measured. The thickness of the phantom is calculated by the difference between Dp and Dg. The thicknesses of the cylinder shaped phantom and the rectangular shaped phantom are obtained $10.45 \mathrm{~mm}$ and $17.48 \mathrm{~mm}$ by this LDS 
measurement.

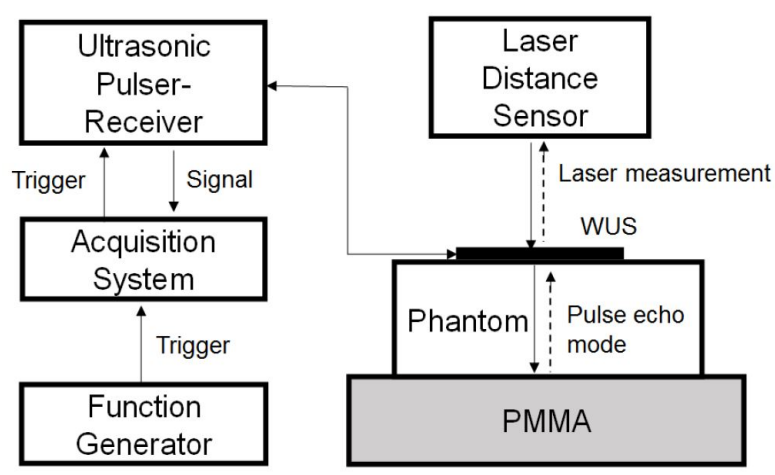

Figure 4.3: An experimental configuration for accuracy evaluation of the thickness estimation using a soft tissue-mimicking phantom and LDS as a golden standard.

After that, the WUS is placed on top of the phantom. The WUS pulse-echo mode measurement set up is as shown in Figure 4.3. The gelatin-based phantom and the PMMA plate are used to mimic soft tissue and bone, respectively. Since, the PMMA material has a relative high density $\left(1.17-1.20 \mathrm{~g} / \mathrm{cm}^{3}\right)$ and acoustic impedance ( $\approx 3.25$ MRayl) than soft tissues [99,100], the boundary between the phantom and PMMA plate has a high reflection coefficient of 0.335 calculated from Equation 2.5. As a result, approximately $33.5 \%$ of the ultrasound signal will be reflected at the boundary between the phantom and the PMMA plate.

Figure 4.4 shows the RF signals obtained from the cylinder shaped phantom (a) and rectangular shaped phantom (b). In the RF signal, the echo on the left is received by the WUS after completing a full round trip within the phantom. The echo on the right is obtained when the received ultrasound echo makes another round trip within the phantom and received by the WUS again. The time delay $(\Delta \mathrm{t})$ is determined by the difference between the first and second round trip echoes as shown in Figure 4.4. The average time delay obtained from cylinder $\left(\Delta \mathrm{t}_{c}\right)$ and rectangular shaped $\left(\Delta \mathrm{t}_{r}\right)$ phantoms are $13.78 \mu \mathrm{s}$ and $22.98 \mu \mathrm{s}$, respectively. By inputting the time delay and 


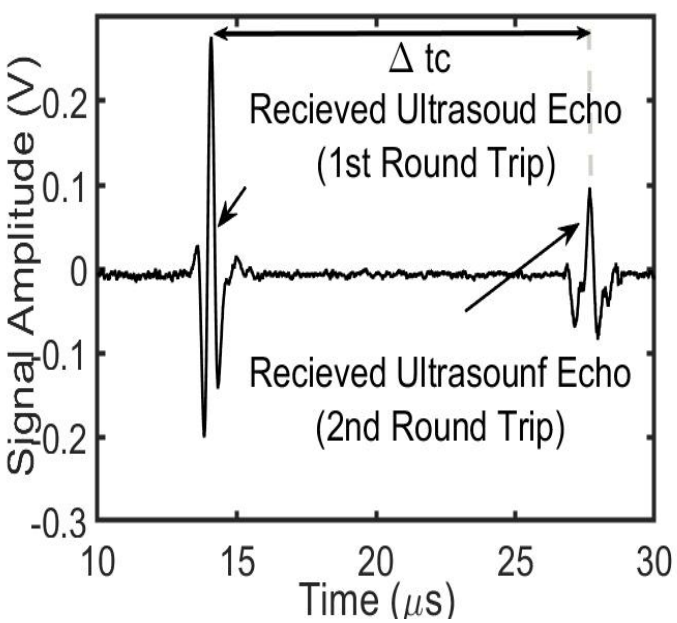

(a)

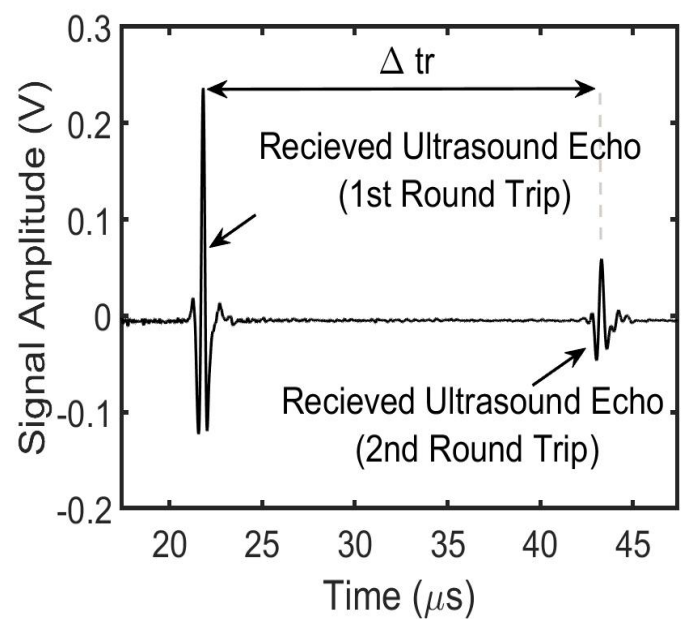

(b)

Figure 4.4: The raw RF signal obtained from the cylinder shaped phantom (a) and rectangular shaped phantom (b).

phantom total thickness into Equation 4.1, the average estimated phantom ultrasound velocity $\left(\mathrm{v}_{p}\right)$ of the cylinder and rectangular shaped phantoms are $1516.55 \mathrm{~m} / \mathrm{s}$ and $1520.07 \mathrm{~m} / \mathrm{s}$ at room temperature $\left(21^{\circ} \mathrm{C}\right)$, respectively. The resultant ultrasound velocity matches with the 12 wt \% gelatin concentration obtained from the literature $[66,90,91,101]$

The acoustic attenuation shows the reduction in ultrasound echo strength when the ultrasound echo propagates in the medium. The acoustic attenuation $(\mu)$ for the phantoms can be estimated by finding the peak to peak amplitude of the 1st echo $\left(A p_{1}\right)$ and 2 nd echo $\left(A p_{2}\right)$, the distance of the ultrasound traveled $(\mathrm{d})$ and the ultrasound frequency (f). The following equation

$$
\text { Attenuation }=\frac{10 \log _{10}\left(\frac{A p_{2}}{A p_{1}}\right)}{d \cdot f}
$$

calculates the acoustic attenuation of the phantom with the unit of $\mathrm{dB}(\mathrm{cm} \cdot \mathrm{MHz})^{-1}$. The calculated acoustic attenuation for the cylinder and rectangular shaped phantoms 
are 0.66 and $0.52 \mathrm{~dB}(\mathrm{~cm} \cdot \mathrm{MHz})^{-1}$, respectively. Acoustic impedance $(Z)$ describe the resistance of the ultrasound pulse when it is passing through the medium, which is calculated by equation

$$
Z=\rho_{p} \cdot v_{p}
$$

where $\rho_{p}$ is the density of the phantom and $v_{p}$ is the ultrasound velocity of the phantom. The density of the phantom is calculated by equation

$$
\rho=W / M
$$

where $W$ is the weight of the phantom and $M$ is the volume of the phantom. The weights of the cylinder and rectangular shaped phantoms, which are measured by an electrical balance, are $140.04 \mathrm{~g}$ and $150.84 \mathrm{~g}$, respectively. Table 4.3 shows the density, ultrasound velocity, acoustic impedance and acoustic attenuation estimated from the cylinder and rectangular shaped phantoms [66,90,91, 101].

Table 4.3: Acoustic properties of the gelatin-based materials and biological tissues $[53,76,77,81]$.

\begin{tabular}{|c|c|c|c|c|}
\hline Material & $\begin{array}{c}\text { Ultrasound } \\
\text { Velocity } \\
(\mathrm{m} / \mathrm{s})\end{array}$ & Density & $\begin{array}{c}\text { Ultrasound } \\
\text { Attenuation } \\
\left(\mathrm{kg} / \mathrm{m}^{3}\right)\end{array}$ & $\begin{array}{c}\text { Acoustic } \\
\text { Impedance } \\
(\mathrm{dB} /(\mathrm{cm} \cdot \mathrm{MHz}))\end{array}$ \\
\hline $\begin{array}{c}\text { Cylinder } \\
\text { Phantom }\end{array}$ & 1516.55 & 1066.78 & 0.66 & 1.62 \\
\hline $\begin{array}{c}\text { Rectangular } \\
\text { Phantom }\end{array}$ & 1520.07 & 1059.29 & 0.52 & 1.61 \\
\hline $\begin{array}{c}\text { Soft Tissue } \\
\text { (Average) }\end{array}$ & 1540.00 & 1043.00 & $0.54-1.00$ & $1.00-1.63$ \\
\hline
\end{tabular}

The fabricated gelatin phantoms are changeable in shape and thickness, which meet the basic requirement for the simulation experiment. In addition, both cylinder 
4.2 Selection of the DSP Techniques for Thickness Measurement Based on Accuracy

Evaluation

and rectangular soft tissue mimicking phantoms have a similar ultrasound velocity $(\approx 1540 \mathrm{~m} / \mathrm{s})$, acoustic attenuation $\left(\approx 0.54 \mathrm{~dB}(\mathrm{~cm} \cdot \mathrm{MHz})^{-1}\right)$ and acoustic impedance (1.0-1.63 MRayl) to average human soft tissue. Therefore, the phantoms are suitable to be used in the following simulation experiments $[66,90,91,101]$. The calculated ultrasound velocity of the phantoms are used in section 4.2 and 4.3 to estimate the thickness changes of the phantom during the application and release of an external force.

\subsection{Selection of the DSP Techniques for Thickness Measurement Based on Accuracy Evaluation}

Several DSP techniques in thickness measurement are discussed in Chapter 3, including peak detection, cross-correlation and quadrature demodulation. To ensure the accuracy of the WUS thickness measurement system, each aforementioned DSP techniques are performed in the following phantom experiment and the thickness results are compared with the golden standard. The technique with the highest accuracy will be chosen for the in-vivo experiment.

\subsubsection{Experimental Configuration for Single Twitch Phan- tom Simulation}

The WUS pulse-echo mode measurement set up is as shown in Figure 4.3. The gelatin-based phantom and the PMMA plate are used to mimic soft tissue and bone, respectively. Since the PMMA material has a relative high density $\left(1.17-1.20 \mathrm{~g} / \mathrm{cm}^{3}\right)$ and acoustic impedance (3.25 MRayl) that are higher than soft tissues [99,100], the boundary between the phantom and PMMA plate has a high reflection coefficient of 
4.2 Selection of the DSP Techniques for Thickness Measurement Based on Accuracy

Evaluation

0.335, calculated by Equation 2.5. In order to evaluate the accuracy of the thickness measurement by the WUS, a LDS is used (Model: LK-H055K, Keyence, Japan) as a gold standard [102]. Since the WUS maximum resolution is $0.18 \mu \mathrm{m}$ by the phase tracking method, the LDS $(0.025 \mu \mathrm{m}$ resolution) provides approximately 7 times higher resolution than the WUS measurement system. The original cylinder shaped phantom thickness is around $10.447 \mathrm{~mm}$ and the maximum thickness changes due to a squeezing force applied by fingers is measured around $0.2 \mathrm{~mm}$. The optimal measurement distance between the LDS and the object in interest is called the reference distance, which the LDS is placed approximately $46.4 \mathrm{~mm}$ away from the measuring object. The optimal measurement range of the LDS is $+/-5 \mathrm{~mm}$ of the reference distance in which the LDS is sufficient to capture the thickness changes of the phantom. The LDS accuracy is $1 \mu \mathrm{m}$ within the optimal measurement range [102]. Therefore, the LDS is eligible as a golden standard in the phantom experiments. The operation of the LDS is further discussed in the Appendix.

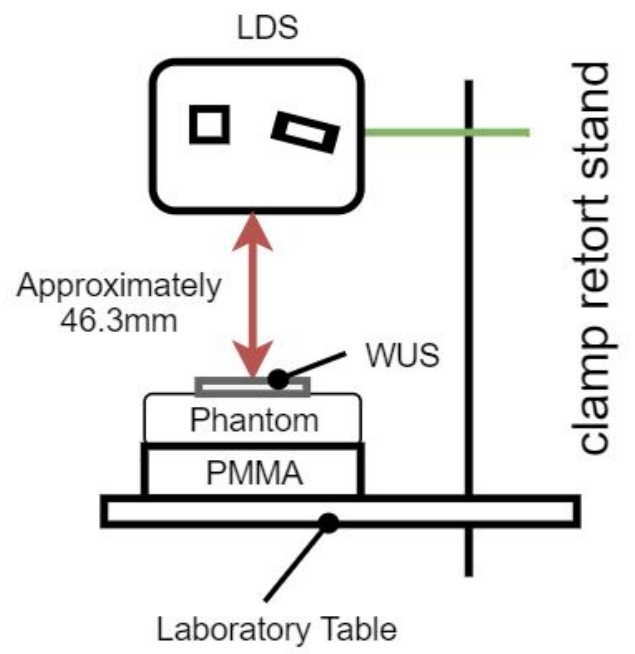

Figure 4.5: The experimental configuration of the $L D S$ in the phantom experiment.

The cylinder shaped phantom is used, and it has a $40 \mathrm{~mm}$ diameter with a thick- 
4.2 Selection of the DSP Techniques for Thickness Measurement Based on Accuracy Evaluation

ness of $10.45 \mathrm{~mm}$. discussed in section 4.1.3. The dimension of the phantom is chosen to allow thickness changes through a squeezing force applied by fingers. When the sides of the phantom are squeezed by fingers, the thickness at the center of the phantom increases. The phantom thickness will slowly decrease when the applied force is removed. The WUS is placed at the center of the top surface of the cylinder shaped phantom. A coupling gel (Aquasonic 100 ultrasound transmission gel, Parker Laboratories Inc, Airfield, NJ, USA) is applied to the WUS and the phantom [103-105]. The LDS is held by the clamp retort stand and the laser head is atop the WUS. To ensure the LDS and the WUS are measuring the same degree of phantom thickness changes, the laser beam is pointing at the center of the active area of the WUS, as shown in Figure 4.5.

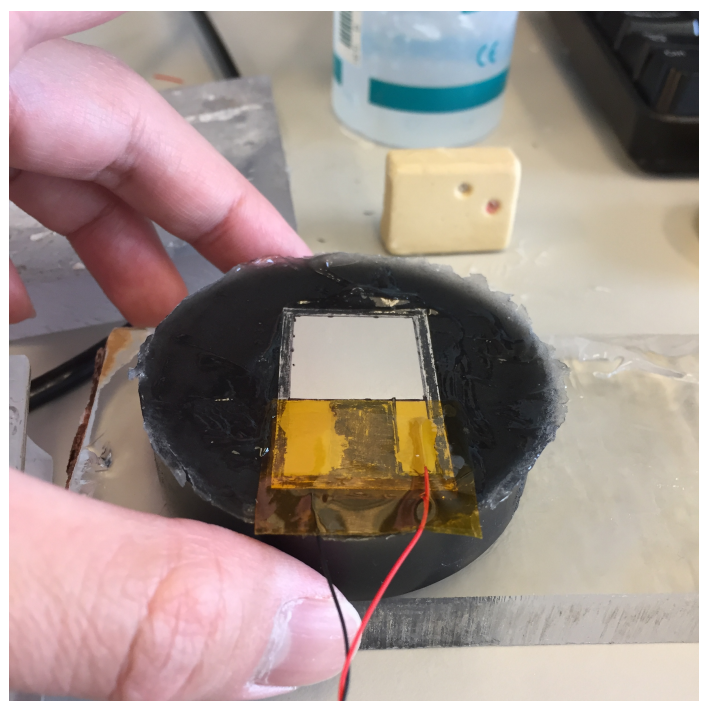

Figure 4.6: An image of the manual squeeze phantom experiment with the cylinder shaped phantom.

An estimated phantom ultrasound velocity of $1516.55 \mathrm{~m} / \mathrm{s}$ is used to calculate the distance in the pulse-echo mode. To produce the thickness changes of the phantom, the phantom is squeezed by fingers gently for 4 seconds then slowly released. An image of the manual squeeze phantom experiment is shown in Figure 4.6. The WUS 
4.2 Selection of the DSP Techniques for Thickness Measurement Based on Accuracy

Evaluation

is employed in measuring the changes in the thickness of the phantom, and the LDS is employed in measuring the displacement between the laser head and the WUS sensor. The raw ultrasound RF signals obtain from the WUS are pre-processed by 5 frames moving averaging filter in order to improve the SNR. A moving averaging of 5 frames at the frame rate of $1000 \mathrm{~Hz}$ is equivalent to the frame rate of $200 \mathrm{~Hz}$, which is able to capture the phantom thickness changes squeezed by fingers. 5 frames moving averaging is efficient to remove the random electrical noise caused by the ultrasonic system. The more detail of the effect of the moving averaging is discussed in Chapter 5.1. After that, three signal processing techniques, such as $\mathrm{P} / \mathrm{D}, \mathrm{C} / \mathrm{C} /$ and $\mathrm{Q} / \mathrm{D}$, are compared for the thickness measurement accuracy. The peak detection technique determines the TOF by finding the maximum peak of the reflected ultrasonic signal. In the cross-correlation technique, the difference of the TOF is obtained by finding the best correlation coefficient between the reflected ultrasonic signals in the two consecutive frames. Then, the thickness is calculated using the estimated TOF and a known ultrasound speed in the specimen (Equation 3.2). Quadrature demodulation is used to recover the phase shift from the RF signals by modulating with a reference signal. This phase shift is induced to RF signals due to the change in time shift of the ultrasound echo. By applying the phase shift to the phase tracking Equation 3.4 the displacement changes $(\Delta \mathrm{d})$ on the consecutive frames is calculated. The thickness estimation is then calculated by accumulating the displacement changes as shown in Equation 3.5. Both LDS measurement and ultrasonic acquisition systems have a frame rate of $1 \mathrm{~ms}$; however, they do not share the same clock cycle.

\subsubsection{Comparison of the DSP Techniques}

$$
R M S E=\sqrt{\frac{\sum_{i=1}^{k}(d x(i)-d y(i))^{2}}{k}}
$$


4.2 Selection of the DSP Techniques for Thickness Measurement Based on Accuracy Evaluation

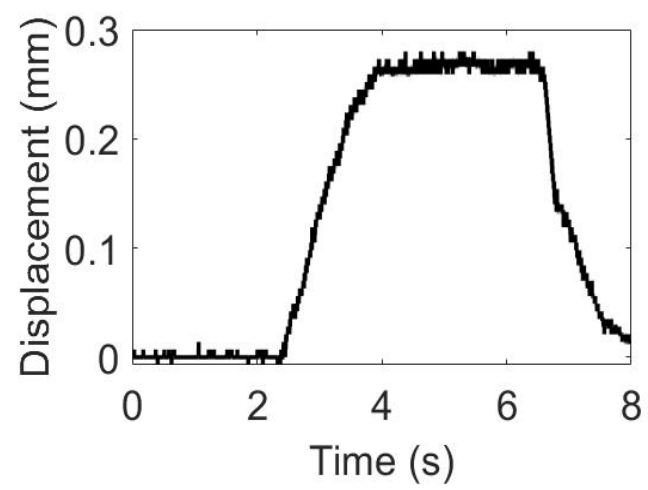

(a)

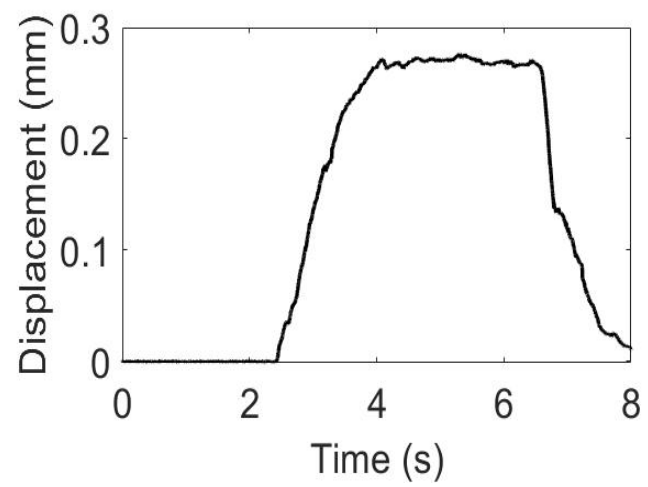

(c)

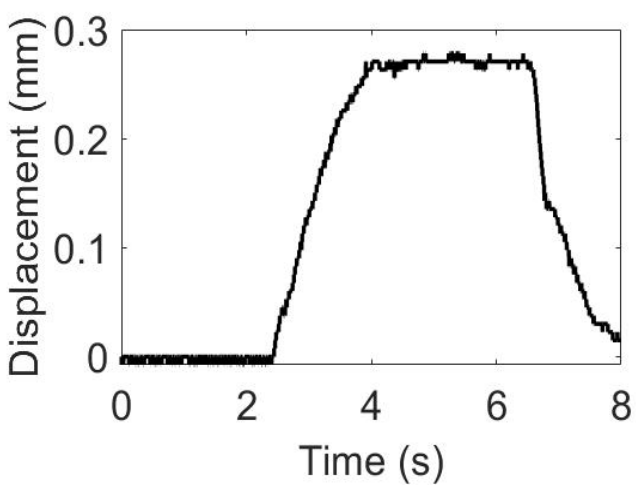

(b)

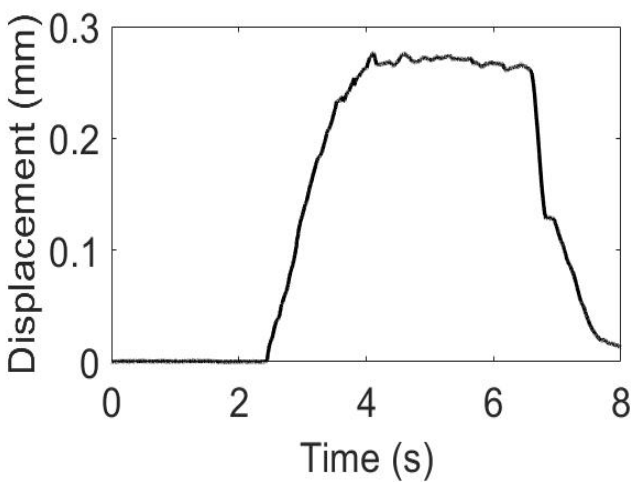

(d)

Figure 4.7: Phantom thickness changes caused by fingers squeeze calculated by peak detection (a), cross-correlation (b), quadrature demodulation (c) and LDS measurement (d).

Figure 4.7 shows the results of the ultrasonic displacement estimation methods (Figures 4.7 (a-c)) with comparison of the LDS measurement result (Figure 4.7 (d)). Root mean square error (RMSE) is calculated using the Equation 4.5 where the thickness estimated from the ultrasonic signal processing techniques $(d x)$ is subtracted from the thickness measurement obtained by the LDS measurement $(d y)$. $i$ corresponds to the frame index and $k$ is the total number of frames during the acquisition. Table 4.4 shows the RMSE for the three displacement estimation methods. RMSE for peak detection method, cross-correlation method, and phase tracking method are 4.0, 3.3 
and $2.0 \mu \mathrm{m}$, respectively. The quadrature demodulation and phase tracking method provide the least RMSE. Therefore, the quadrature demodulation and phase tracking technique are used in the following phantom experiments and in-vivo experiments.

Table 4.4: The comparison between each displacement estimation methods.

\begin{tabular}{|c|c|c|c|}
\hline $\begin{array}{c}\text { Ultrasound Signal } \\
\text { Analysis Method }\end{array}$ & $\begin{array}{c}\text { Peak } \\
\text { Detection }\end{array}$ & $\begin{array}{c}\text { Cross- } \\
\text { Correlation }\end{array}$ & $\begin{array}{c}\text { Quadrature } \\
\text { Demodulation }\end{array}$ \\
\hline RMSE $(\mu \mathrm{m})$ & 4.0 & 3.3 & 2.0 \\
\hline
\end{tabular}

\subsection{Accuracy Evaluation Using Phantom Experi- mental Environment}

In the previous phantom experiments, the WUS has demonstrated the ability in monitoring the thickness changes of the phantom. In addition, the phase tracking method is selected as the DSP technique to estimate thickness change in the following phantom and in-vivo experiments. In this section, the stability of the WUS measurement system is evaluated by comparing the thickness changes with the LDS measurement in a mechanical simulation which mimic the muscle contraction evoked by EMS. In this phantom experiment, an electrical motor is used to provide a mechanical force to the phantom so that the thickness of the phantom changes due to the additional force. Besides, by controlling the rotation speed of the electrical motor, muscle contraction evoked by various EMS frequencies are simulated. In total, there are seven muscle contraction simulations in various stimulation rates (2 to $12 \mathrm{~Hz}$ ) which are conducted in this experiment. The experimental set up of the muscle contraction evoked by EMS simulation will be presented in Section 4.3.1. This experiment is divided into two parts which are the accuracy evaluation of an object in stable position and in mo- 
tion. The repeatability of the thickness estimation by the WUS system is evaluated by measuring the thickness of a stable object. Where the accuracy of the thickness measurement is obtained by measuring the thickness of an object while physically changing in thickness.

\subsubsection{Experimental Setup for Thickness Measurement Accu- racy Evaluation Using a Phantom}

In this experiment, a new phantom is developed to simulate the muscle contraction evoked by EMS with various pulse rates. We named this experiment as mechanical simulation. The rectangular shaped gelatin phantom is constructed from the same material composition as indicated in section 4.1. In order to provide uniform pressure to the phantom, a mold with $40 \mathrm{~mm}$ width, $40 \mathrm{~mm}$ length and $20 \mathrm{~mm}$ height is used to construct the phantom. The ultrasound velocity of the rectangular shaped phantom is estimated as $1520.07 \mathrm{~m} / \mathrm{s}$ as shown in Section 4.1.3. In total, seven muscle contraction simulations are performed with motor rotational frequencies of $0.5 \mathrm{~Hz}, 1 \mathrm{~Hz}, 2 \mathrm{~Hz}, 4$ $\mathrm{Hz}, 6 \mathrm{~Hz}, 10 \mathrm{~Hz}$ and $12 \mathrm{~Hz}$.

A servo motor controlled by Arduino UNO is used to physically deform the phantom at a different rate. Figure 4.8 shows a photograph of the Servo motor with the white rotation arm. Each side of the rotation arm on the servo motor is $15.1 \mathrm{~mm}$ long, which is the maximum displacement exerted by the motor. To simplify the operation, only one rotation arm is used to deform the phantom.

In the experimental setup, a piece of thin PMMA plate is placed between the rotation arm and the phantom shown in Figure 4.9. A fixed PMMA plate is placed on the opposite side of the servo motor. The purpose of the PMMA plates is to ensure a uniform force is applied to the phantom. The force exerted from the motor rotation 


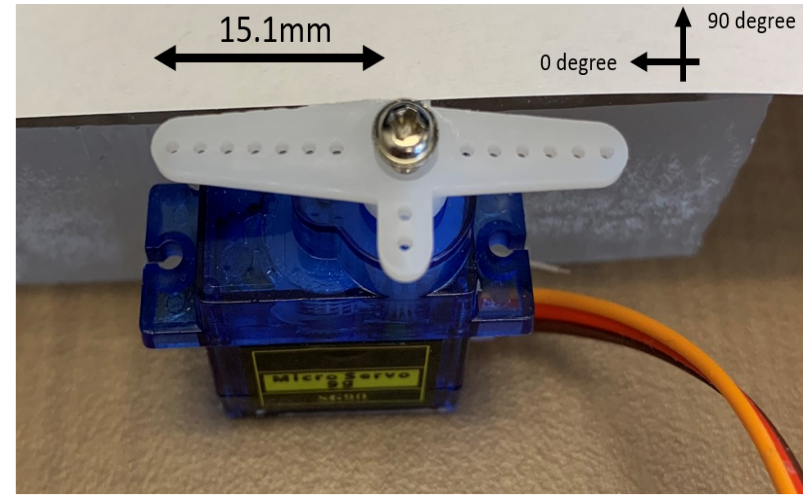

Figure 4.8: A photograph of the servo motor used in the mechanical simulation.

arm deforms the shape of the phantom from the side, which results in the change of the vertical thickness of the phantom due to its elasticity. The following provides the detailed explanation in selecting the angular position and angular velocity of the Servo motor through controlling the Arduino.

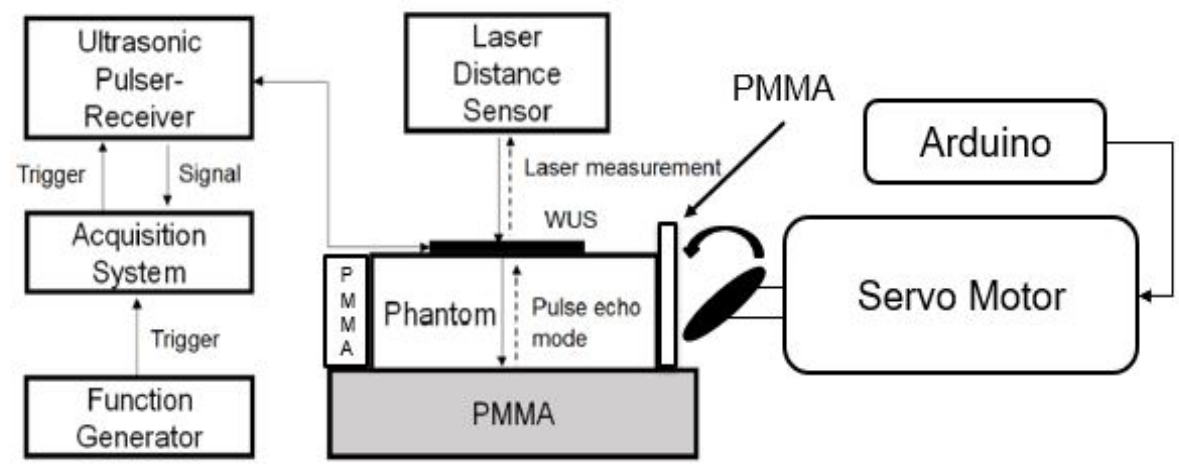

Figure 4.9: The phantom experiment configuration for the mechanical simulation. 


\subsubsection{Simulations for Unfused Muscle Contraction in Low EMS Fre- quencies}

In a single muscle twitch evoked by a single electrical stimulus, the muscle contracts once and then relaxes completely as shown in Figure 2.3. To simulate the thickness changes of a synchronized single muscle twitch, the servo motor rotates from $0^{\circ}$ to a designated degree and then quickly returns to $0^{\circ}$. The change in angular position of the rotation arm will push the phantom to change its thickness in order to stimulate the contraction of the muscle. To imitate the muscle relaxation, the rotation motor immediately returns to $0^{\circ}$ to allow the phantom to return to the original shape gradually due to its elastic property. The top view of the rotation arm angular position of the single twitch contraction in latent, contraction and relaxation period is shown in Figure 4.10. $\Delta \mathrm{L}$ represents the change in phantom length due to the mechanical

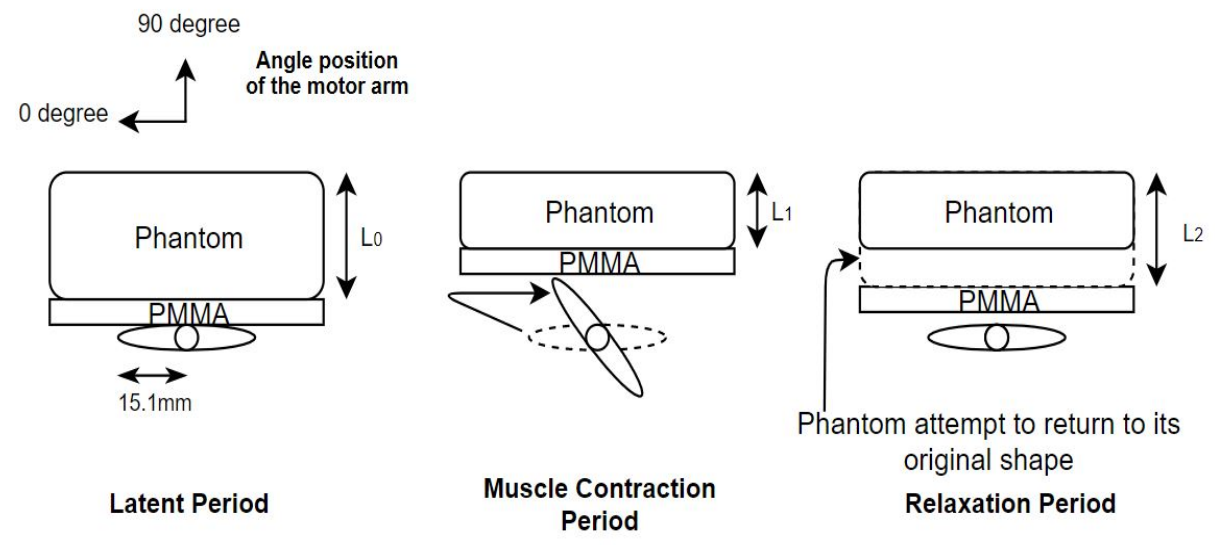

Figure 4.10: The phantom experiment configuration for the muscle contraction evoked by $E M S$.

force applied from the servo motor. For example, when the angular position is moving from $0^{\circ}$ to $50^{\circ}$ in one cycle, there are 50 steps/angles in total. Such that, the number of steps/angles $(S)$ is equal to 50 . The angular velocity $(w)$ is the speed of 
the rotational arm which has a unit of ms/angle. For example, the angular velocity of $5 \mathrm{~ms} /$ angle indicates the time it takes for the servo motor to rotate from the current angular position to the consecutive angular position. The total time it takes for one deformation cycle is equal to $2 \mathrm{x}$ angular velocity $(w) \mathrm{x}$ number of steps/angle $(S)$. Therefore, if the deformation is applied periodically, the estimated motor rotation frequency is calculated by Equation 4.6. Since, the oscillation of the EMS is periodic, the servo motor performs the procedures in Figure 4.11 in a loop to change the thickness of the phantom periodically. The rotational angle and angular velocity for phantom simulation in low frequency $(0.5$ to $2 \mathrm{~Hz})$ are shown in Table 4.5 .

$$
F r=\frac{1}{2 \cdot S \cdot w}
$$

Table 4.5: Angular position and angular velocity setting for the servo motor in low EMS frequency muscle contraction simulation

\begin{tabular}{|c|c|c|c|}
\hline $\begin{array}{c}\text { Muscle } \\
\text { Contraction } \\
\text { Simulation \# }\end{array}$ & $\begin{array}{c}\text { Estimated } \\
\text { Frequency }\end{array}$ & $\begin{array}{c}\text { Angular } \\
\text { Position } \\
(1 \text { cycle })\end{array}$ & $\begin{array}{c}\text { Angular } \\
\text { Velocity }\end{array}$ \\
\hline 1 & $0.5 \mathrm{~Hz}$ & $0^{\circ}$ to $90^{\circ}$ & $10 \mathrm{~ms} /$ angle \\
\hline 2 & $1.0 \mathrm{~Hz}$ & $0^{\circ}$ to $90^{\circ}$ & $6 \mathrm{~ms} /$ angle \\
\hline 3 & $2.0 \mathrm{~Hz}$ & $0^{\circ}$ to $50^{\circ}$ & $6 \mathrm{~ms} /$ angle \\
\hline
\end{tabular}

\subsubsection{Simulations for Unfused and Fused Muscle Contraction in High EMS Frequencies}

The muscle cannot fully relax when experiencing a high firing pulse rate EMS. As a result, wave summation (Chapter 2.2.2) occurs when the stimulus firing rate is higher than the duration of a single twitch and the muscle tension gradually increases [38]. For instance, Figure 4.11 shows a muscle thickness plot evoked by a high pulse 


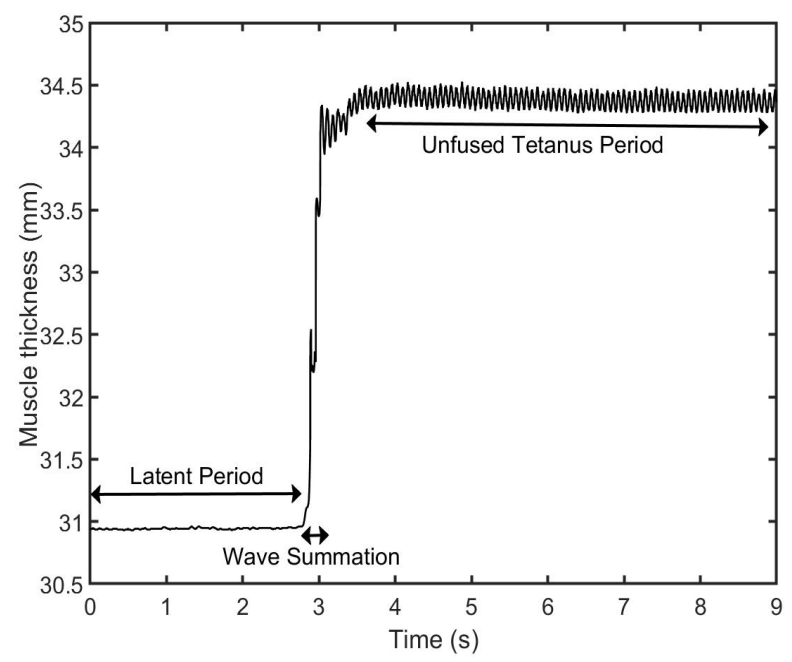

Figure 4.11: Muscle thickness measured by ultrasound measurement obtained from a tricep muscle electrically evoked at $14 \mathrm{~Hz}$.

rate EMS and the labels indicating contraction period, wave summation period and unfused tetanus period. During the unfused tetanus period, the muscle thickness changes is significantly smaller in comparison to the wave summation period. The number of muscle twitches within one second is equivalent to the applied EMS frequency in the unfused tetanus period. As the EMS pulse rate increases, the relaxation time is further reduced in which the tetanus fluctuation or muscle thickness changes is further reduced. Since wave summation does not exist in low EMS frequency, the wave summation period is added in the setting of angular position and angular velocity through Arduino in high frequency $(4-12 \mathrm{~Hz})$ muscle contraction simulation. Figure 4.12 shows an example of the motor rotation arm motion in latent, wave summation and unfused tetanus period. In order to mimic the skeletal muscle response to high EMS firing frequency, the servo motor is programmed to perform the following instructions:

1. A $1 \mathrm{~s}$ delay after the start of the acquisition to imitate the stable muscle (latent period) before receiving a stimulus. 
2. Followed by latent period, the servo motor is programmed to hit the phantom to create a large displacement to mimic the wave summation period. Unlike the unfused tetanus period, there are no obvious muscle relaxation observed during the wave summation period. Therefore, the angular position of the motor will not return to $0^{\circ}$.

3. After the wave summation period, the servo motor vibrates at the corresponding frequency to mimic the unfused tetanus period. The procedure of the unfused tetanus period is identical to the periodic rotation motion in the low frequency as shown in Figure 4.10). The rotational frequency (mimic EMS frequency) is calculated by Equation 4.6.

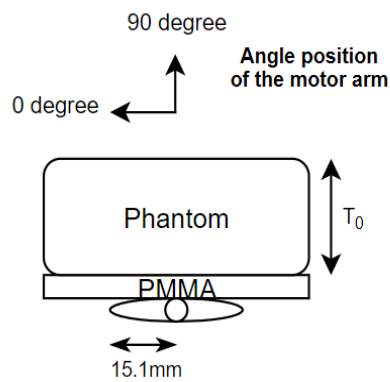

Latent Period

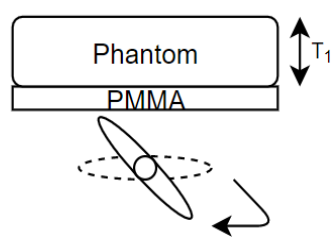

Wave Summation Period

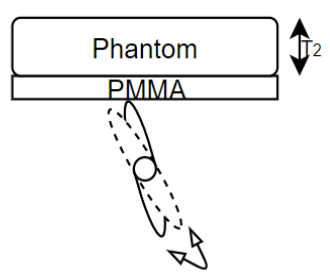

Unfused Tetanus

Period

Figure 4.12: Procedure of the angular position setting of the rotation arm in high EMS frequency muscle contraction simulation.

Table 4.6 shows the selection of angular position and angular velocity of the servo motor in 4 to $12 \mathrm{~Hz}$ reputations EMS frequency muscle contraction simulation.

\subsubsection{Data Pre-processing in Mechanical Simulation}

In pre-processing, the smoothing averaging filter is applied to the raw RF signal, in order to remove the noise and increase the SNR. 3 frames moving averaging corre- 


\begin{tabular}{|c|c|c|c|c|c|}
\hline & \multicolumn{2}{|c|}{ Wave Summation Period } & \multicolumn{3}{c|}{ Unfused Tetanus Period } \\
\hline $\begin{array}{c}\text { Muscle } \\
\text { Contraction } \\
\text { Simulation }\end{array}$ & $\begin{array}{c}\text { Angular } \\
\text { Position }\end{array}$ & $\begin{array}{c}\text { Angular } \\
\text { Velocity } \\
\text { (one direction) }\end{array}$ & $\begin{array}{c}\text { Angular } \\
\text { Position } \\
\text { (vibrate) }\end{array}$ & $\begin{array}{c}\text { Angular } \\
\text { Velocity } \\
\text { (continuous) }\end{array}$ & $\begin{array}{c}\text { Estimated } \\
\text { Frequency }\end{array}$ \\
\hline 4 & $0^{\circ}$ to $50^{\circ}$ & $10 \mathrm{~ms} /$ angle & $50^{\circ}$ to $57^{\circ}$ & $15 \mathrm{~ms} /$ angle & $4.0 \mathrm{~Hz}$ \\
\hline 5 & $0^{\circ}$ to $60^{\circ}$ & $10 \mathrm{~ms} /$ angle & $60^{\circ}$ to $64^{\circ}$ & $15 \mathrm{~ms} /$ angle & $6.0 \mathrm{~Hz}$ \\
\hline 6 & $0^{\circ}$ to $70^{\circ}$ & $10 \mathrm{~ms} /$ angle & $70^{\circ}$ to $74^{\circ}$ & $10 \mathrm{~ms} /$ angle & $10.0 \mathrm{~Hz}$ \\
\hline 7 & $0^{\circ}$ to $80^{\circ}$ & $10 \mathrm{~ms} /$ angle & $80^{\circ}$ to $83^{\circ}$ & $10 \mathrm{~ms} /$ angle & $12.0 \mathrm{~Hz}$ \\
\hline
\end{tabular}

Table 4.6: Angular position and angular velocity setting for the servo motor in high EMS frequency muscle contraction simulation.

sponds to $3 \mathrm{~ms}$ of averaging window in the experiment configuration. The original acquisition system has a frame rate of $1000 \mathrm{~Hz}$. However, after applying 3 frames averaging the frame rate is decreased from $1000 \mathrm{~Hz}$ to $333.3 \mathrm{~Hz}$. In order to recover all the Fourier components, the frame rate is required to have at least twice the rotational frequency of the servo motor. The highest rotational frequency in this phantom experiment is $12 \mathrm{~Hz}$, therefore the acquisition system is able to capture the thickness changes due to the rotation of the motor. In addition, the minimum rotation time of the servo motor is $6 \mathrm{~ms}$, which is corresponding to approximately $166.7 \mathrm{~Hz}$. With the frame rate of $333.3 \mathrm{~Hz}$, the Fourier components including the phase of the phantom thickness changes due to the servo motor can be captured. Quadrature demodulation is then applied to the filtered RF signal to obtain the phase of ultrasound RF signals. After that, the phase difference between the two consecutive RF signals are converted into thickness changes. A baseline is then set by calculating the average displacement during the stable/latent period in the WUS thickness measurement. The baseline is used as a reference to match with the LDS thickness changes measurement in the displacement amplitude. Finally, the thickness changes measurements between the ultrasound and the LDS measurement are compared. A $2 \mathrm{~Hz}$ rotation frequency (a) and $10 \mathrm{~Hz}$ rotation frequency (b) displacement plots are shown in Figure 4.13. The 
line colored in blue and red are the phantom displacement estimation obtained from the LDS measurement and ultrasound phase tracking method, respectively. Each thickness measurement from mechanical simulation is divided into stable period and dynamic period (wave summation and unfused tetanus period) to evaluate the repeatability and accuracy of the WUS measurement system, respectively.

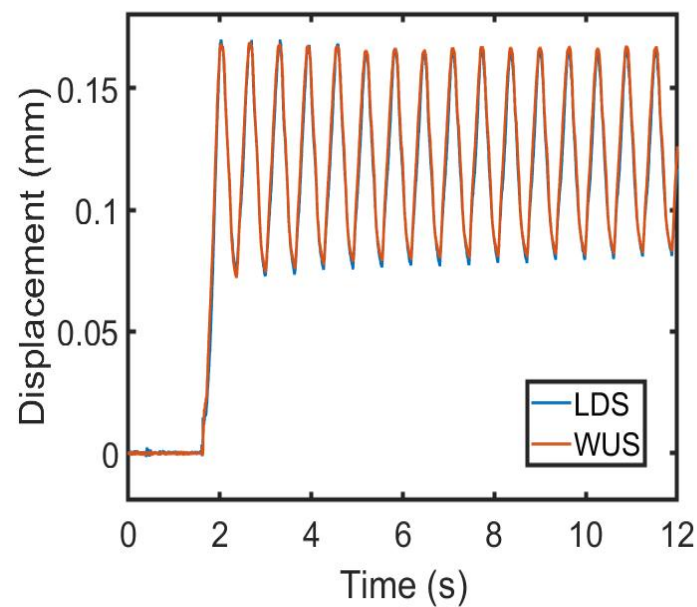

(a)

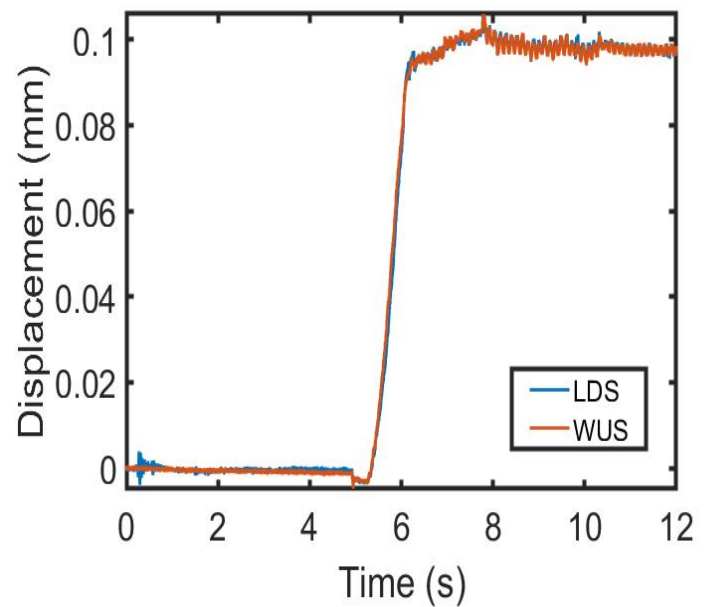

(b)

Figure 4.13: Phantom thickness changes estimated by LDS and ultrasound phase tracking method evoked by $2 \mathrm{~Hz}$ (a) and $10 \mathrm{~Hz}$ (b) motor rotational frequency.

\subsubsection{Repeatability and Accuracy Evaluation for Dynamic Contraction}

Repeatability of the measurement is referring to the variation of the measurement results taken by the same instrument under the same circumstances. Hence, the LDS is not involved in the repeatability test. The repeatability is important because it shows the agreement between each measurement. In the muscle contraction simulation phantom experiment, the thickness measurement in the stable/latent period is analyzed and shown in Figure 4.14. In each muscle simulation, 1000 data points 
are analyzed for the repeatability test. The EMS evoked muscle contraction simulation from left to right corresponds to the stable period in $0.5,1,2,4,6,10$ and $12 \mathrm{~Hz}$ motor rotational frequency. From the box plot, the repeatability of the WUS is approximately $+/-1 \mu \mathrm{m}$. The repeatability test result indicates that the WUS measurement system has thickness estimation resolution in $+/-1 \mu \mathrm{m}$ for the stable phantom.

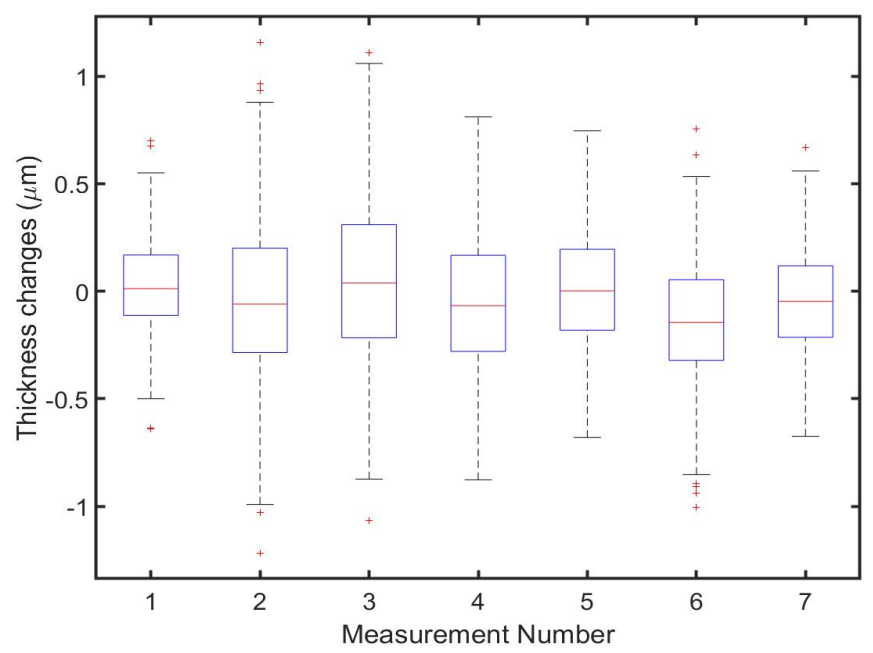

Figure 4.14: Thickness changes on stationary phantom estimated by the WUS measurement System. 1000 data points are analyzed from each muscle simulation.

The LDS is then used to evaluate the accuracy of the WUS measurement system. During the experiment, some environmental motions are observed by the LDS. However, such motion is not observed in the thickness measurement obtained by the WUS. Figure 4.15 shows the environmental motion observed from the LDS during the EMS evoked muscle contraction simulation experiment with rotational frequency of $2 \mathrm{~Hz}$. This motion may come from the motion of the building. Since the vibration of the building does not cause the thickness changes of the phantom, the WUS measurement system may not be affected by such motion. The data where the environmental motion occurs are removed from both LDS and WUS thickness measurements in the 


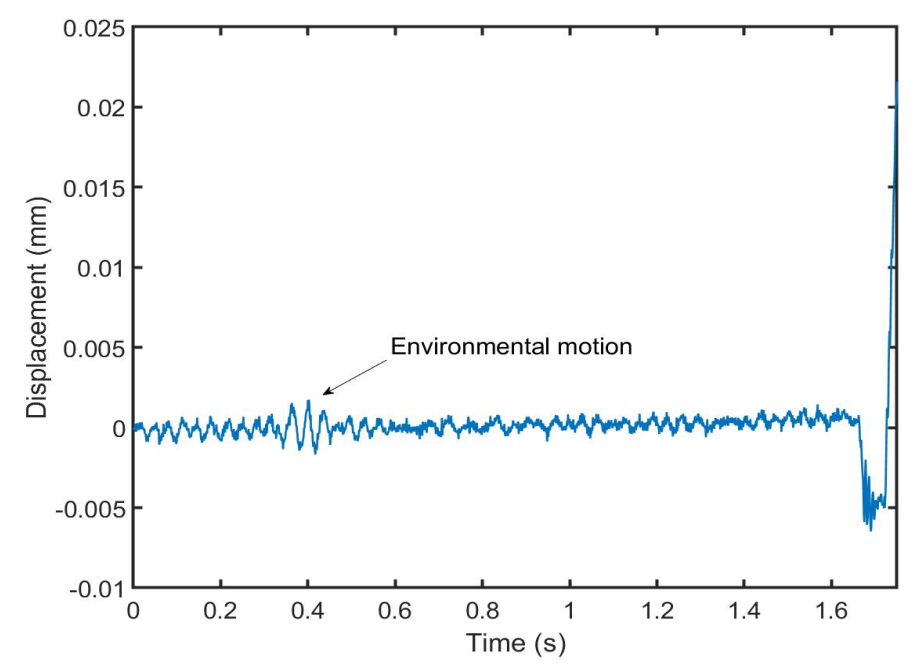

Figure 4.15: The observed environmental motion during muscle contraction simulation \#3 (2 Hz rotational frequency).

following accuracy analysis. 800 data points from each muscle simulations are evaluated in the following accuracy analysis. The RMSE is calculated to compare the difference between the results obtained by the WUS measurement and LDS measurement. The absolute error is the difference of the measured value from the true value. The thickness changes measurement obtained from the WUS measurement system is compared with the LDS system to find the absolute error. Absolute error is defined by

$$
\text { AbsoluteError }=|d x-d y|
$$

where $d x$ is representing the reference value obtained by the LDS system and $d y$ is representing the thickness changes estimated by the WUS measurement system. Table 4.7 shows the RMSE and the maximum absolute error of the WUS thickness changes estimation. It is found that the maximum RMSE error is $0.56 \mu \mathrm{m}$ and the maximum absolute error of $1.79 \mu \mathrm{m}$ at the measurement \#2. The thickness estimation difference between the WUS and LDS may come from the fact that the laser spot 
Table 4.7: The RMSE and maximum absolute error observed from each phantom simulation experiment when the phantom is stable. 800 points are analyzed from each muscle simulation.

\begin{tabular}{|c|c|c|c|c|c|c|c|}
\hline Muscle Contraction Simulation $\#$ & 1 & 2 & 3 & 4 & 5 & 6 & 7 \\
\hline Rotation frequency $(\mathrm{Hz})$ & 0.5 & 1 & 2 & 4 & 6 & 10 & 12 \\
\hline RMSE $(\mu \mathrm{m})$ & 0.46 & 0.56 & 0.47 & 0.33 & 0.25 & 0.27 & 0.32 \\
\hline Maximum Absolute Error $(\mu \mathrm{m})$ & 1.63 & 1.79 & 1.69 & 1.45 & 1.33 & 1.27 & 1.61 \\
\hline
\end{tabular}

diameter is $50 \mu \mathrm{m}$ X $2000 \mu \mathrm{m}$ while the WUS sensing area is $20 \mathrm{~mm}$ by $20 \mathrm{~mm}$. The larger observation area of the WUS provides a more consistent measurement since the tiny motion changes may be removed by the smoothing effect. In this stability experiment, the accuracy of the WUS measurement system is around $1.79 \mu \mathrm{m}$, which is sufficient to measure the thickness changes of the muscle due to muscle contraction.

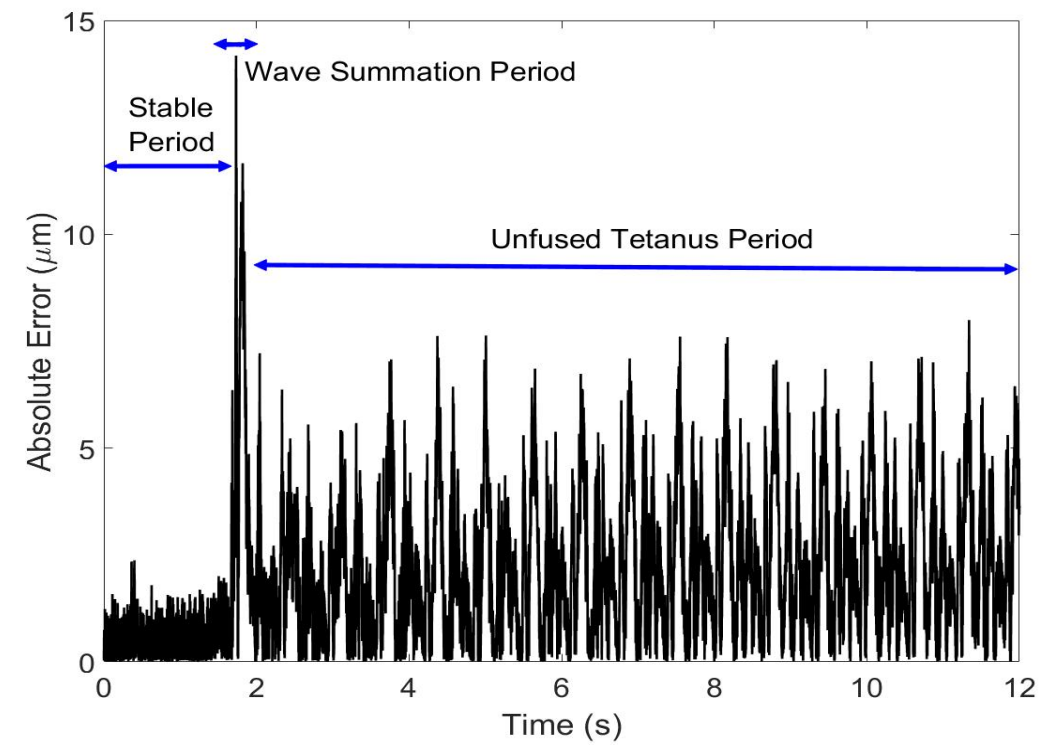

Figure 4.16: Absolute error calculated from muscle contraction simulation \#3 (2 $\mathrm{Hz}$ rotational frequency).

Figure 4.16 shows the absolute error of the muscle contraction simulation at the $2 \mathrm{~Hz}$ rotation frequency. The maximum error occurs in the wave summation period. 
The motor arm makes the initial contact with the PMMA plate, causing the surface of the phantom to change, but not the centre thickness of the phantom. The LDS measures the displacement on the surface of the phantom while the ultrasound is measuring the thickness of the phantom. Therefore, a discrepancy is created between the two measurements. Another possible reason in creating such measurement error is due to the estimated ultrasound velocity in the phantom. The phase tracking method is mainly relying on the ultrasound velocity and the phase changes in order to calculate the thickness changes in the phantom. If the estimated ultrasound velocity is different from the actual ultrasound velocity of the phantom, such measurement error may occur. For example, if the ultrasound velocity is underestimated, the calculated displacement will be smaller than the actual thickness changes and vise versa. One observed evidence from the absolute error graph is that the regions with the high errors are located at the peak and the valley of the displacement plot. Figure

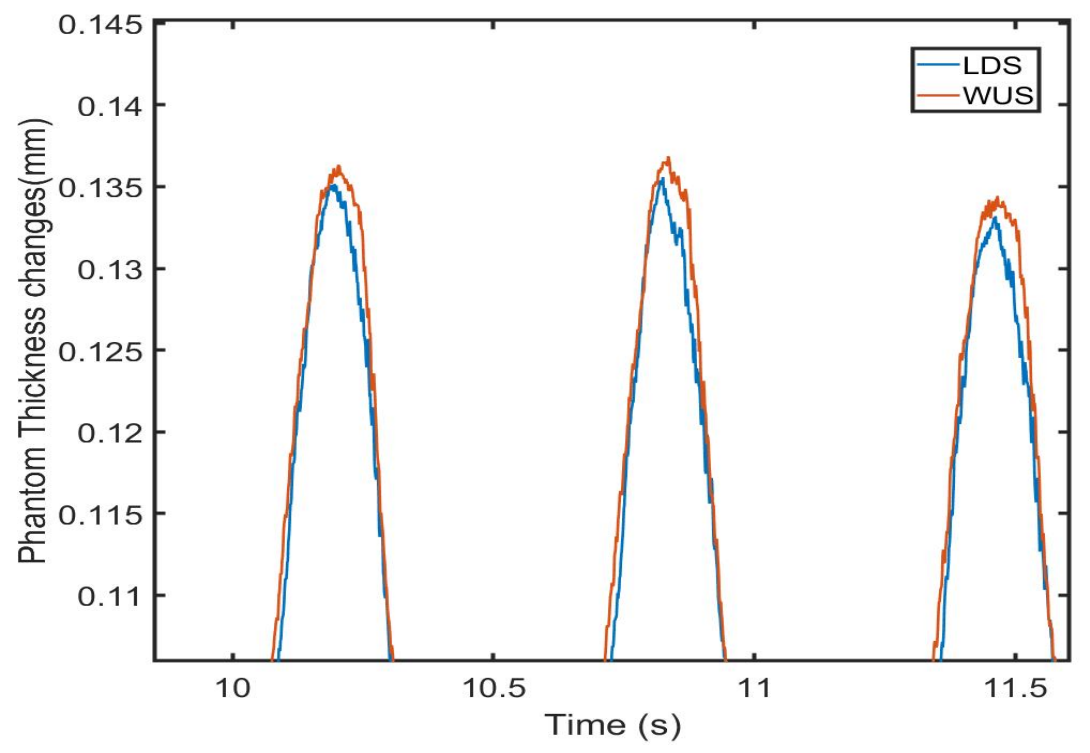

Figure 4.17: Enlarged Portion of Figure 4.14 (a)

4.17 shows a zoomed image of the phantom thickness changes obtained from muscle 
simulation \#3 $(2 \mathrm{~Hz})$. This shows that the estimated ultrasound velocity used in the phase tracking method is higher than the actual phantom ultrasound velocity in this current muscle simulation. In medical ultrasound imaging, relative tissue thickness changes are measured instead of the absolute thickness changes. It is because the ultrasound velocity in the body is not constant. Different tissues have variation in ultrasound velocity. Through surgical operation, the ultrasound velocity of the tissue can be measured; however, it is seriously harmful to the subject. To remain as a non-invasive method, the average human soft tissue ultrasound velocity $(1540 \mathrm{~m} / \mathrm{s})$ is used to predict the relative thickness changes in medical imaging.

To provide a more consistent accuracy evaluation, only the unfused tetanus period (where the phantom thickness changes is periodic) is considered. In this accuracy evaluation, 8000 data points are analyzed from each muscle simulation. Table 4.8 shows the RMSE and maximum absolute error of the muscle stimulation in various frequencies (from $0.5 \mathrm{~Hz}$ to $12 \mathrm{~Hz}$ ) during the unfused tetanus period. Figure 4.18 shows the box plot of the absolute error in various frequencies. The RMSE is 1.65 $\mu \mathrm{m}$ and the maximum absolute error is $7.2 \mu \mathrm{m}$.

Table 4.8: The RMSE and maximum absolute error observed from each phantom simulation experiment during unfused tetanus period. 8000 points are analyzed from each muscle simulation.

\begin{tabular}{|c|c|c|c|c|c|c|c|}
\hline Muscle Contraction Simulation \# & 1 & 2 & 3 & 4 & 5 & 6 & 7 \\
\hline Rotation frequency $(\mathrm{Hz})$ & 0.5 & 1 & 2 & 4 & 6 & 10 & 12 \\
\hline RMSE $(\mu \mathrm{m})$ & 1.68 & 2.31 & 3.18 & 1.32 & 0.86 & 0.98 & 1.33 \\
\hline Maximum Absolute Error $(\mu \mathrm{m})$ & 4.91 & 4.79 & 7.20 & 4.31 & 2.80 & 2.13 & 1.94 \\
\hline Average thickness change $(\mu \mathrm{m})$ & 191 & 125 & 91 & 40 & 12 & 4 & 3 \\
\hline
\end{tabular}

The displacement plot at $2 \mathrm{~Hz}$ has the greatest absolute error, which may be caused by the error in predicting the ultrasound velocity used in the thickness calculation. 


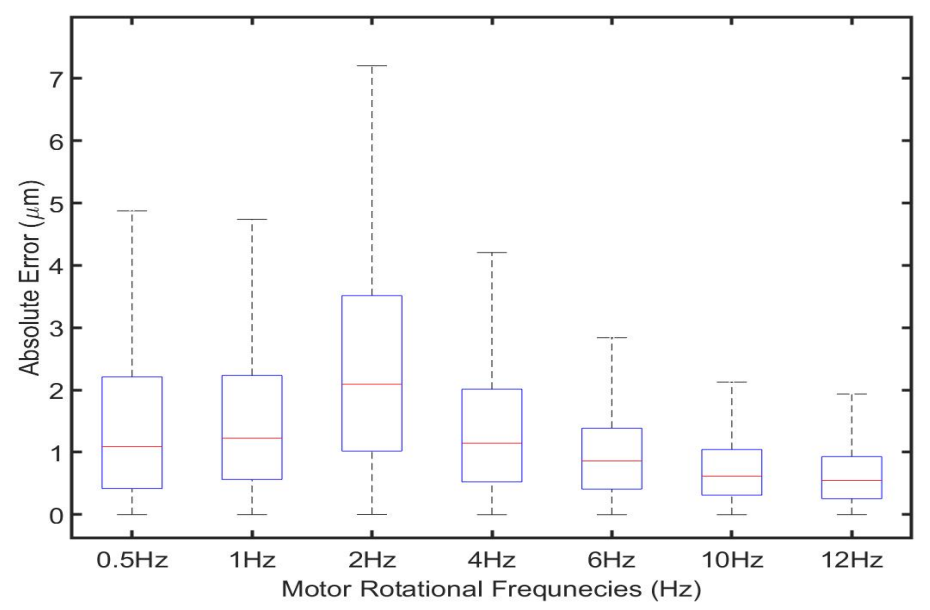

Figure 4.18: Absolute error obtained from various frequencies $(0.5$ to $12 \mathrm{~Hz})$ muscle contraction simulation in unfused tetanus period. 8000 points are analyzed from each muscle simulation.

From the absolute error graph obtained from muscle contraction simulation \#3 (2 $\mathrm{Hz}$ ), the maximum errors occur at the peaks of the maximum displacement, shown in Figure 4.16. Due to the fact that the ultrasound velocity of the object is dependant on temperature, a slight change of the temperature may affect the ultrasound velocity. The light from the LDS laser that is emitted onto the surface of the WUS may indirectly increase the temperature of the surface of the phantom. Parker, et al. investigates the ultrasound speed variation in gelatin solution during a heating phase from $5{ }^{\circ} \mathrm{C}$ to $80{ }^{\circ} \mathrm{C}[106]$. They found that the increase in temperature also increases the ultrasound velocity in the gelatin phantom. For a $10 \mathrm{wt} \%$ gelatin concentration phantom, the ultrasound velocity at $20{ }^{\circ} \mathrm{C}$ and $21{ }^{\circ} \mathrm{C}$ are approximately $1520 \mathrm{~m} / \mathrm{s}$ and $1525 \mathrm{~m} / \mathrm{s}$, respectively [106]. By using these ultrasound velocities to calculate the phantom displacement, we will get $18.392 \mathrm{~mm}$ and $18.453 \mathrm{~mm}$. Therefore, the error due to $1{ }^{\circ} \mathrm{C}$ temperature variation is approximately $60 \mu \mathrm{m}$. Since the emission of the laser is not strong enough to increase the temperature of the entire phantom by $1{ }^{\circ} \mathrm{C}$, a smaller change in temperature may induce such an error. By assuming the linear 
relationship between ultrasound velocity and the temperature change in the gelatin phantom, $1{ }^{\circ} \mathrm{C}$ will cause $60 \mu \mathrm{m}$ error, which corresponds to $7.2 \mu \mathrm{m}$ absolute error. Therefore, the ratio is 0.12 which is $60 \mu \mathrm{m} / 7.2 \mu \mathrm{m}$ and the temperature of phantom is increased by $0.12{ }^{\circ} \mathrm{C}$. Since the ultrasound velocity $(1520 \mathrm{~m} / \mathrm{s})$ is estimated from the beginning of the experiment when the phantom temperature is approximately 21 ${ }^{\circ} \mathrm{C}$, the temperature is increased approximately $0.12{ }^{\circ} \mathrm{C}$ during the experiment.

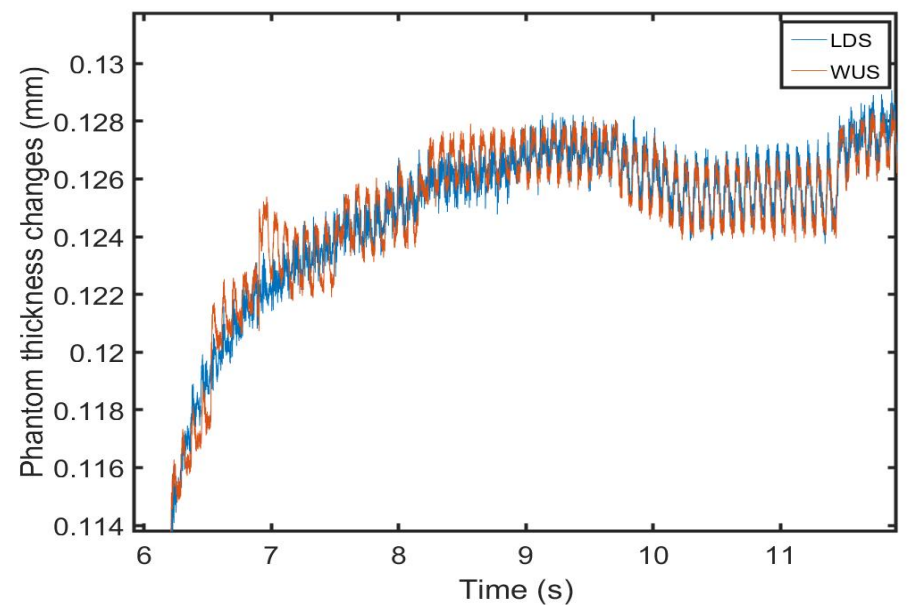

Figure 4.19: Enlarged Phantom thickness changes estimated by LDS and ultrasound phase tracking method evoked at $12 \mathrm{~Hz}$ motor rotational frequency.

In each phantom contraction, the total thickness changes is calculated by subtracting the minimum thickness changes from the peak thickness changes. After that, the average thickness changes can be found by accumulating the thickness changes of each contraction and then dividing by the total number of contractions. Table 4.8 shows the phantom thickness changes with the corresponding absolute error. Although the average absolute error in high phantom oscillation is approximately $1 / 3$ of the phantom average thickness changes, the resultant thickness changes from the WUS has a high agreement with the LDS measurement as shown in Figure 4.19. Therefore, the WUS is capable to detect $3 \mu \mathrm{m}$ thickness changes. 


\section{Chapter 5}

\section{In-vivo Experiments for}

\section{Monitoring Skeletal Muscle}

\section{Contraction Using a Wearable}

\section{Ultrasound Sensor 1}

This chapter provides a detailed procedure in muscle contraction monitoring using the wearable ultrasonic sensor (WUS) developed in our group. The experimental setup and the participants have been described. Analysis has been performed to obtain various muscle contractile parameters for each participant.

\footnotetext{
${ }^{1}$ Contents presented in this chapter have partially been published (or submitted for publication) in [107] by the author.
} 


\subsection{Effect of Moving Averaging on Thickness Mea- surement Accuracy}

The main objective of this thesis research is to develop a procedure in skeletal muscle monitoring using the WUS. In previous section, the accuracy and repeatability of the WUS measurement system are evaluated by performing thickness measurement of the phantoms. However, as the ultrasonic signal travels through the tissue, the amplitude of the signal is attenuated due to scattering, absorption, reflection, and diffraction [90]. Signal-to-noise ratio (SNR) is commonly calculated by dividing the peak to peak amplitude of the echo of interest with that of the noises. Measurement errors might occur due to poor SNR. Moving averaging is one of the preprocessing techniques applied to the acquired raw RF ultrasound signals in order to suppress the noises and increase the SNR. In general, the greater the number of frames averaging, the higher the SNR. To further investigate the relationship of the SNR with the numbers of averaging and the thickness measurement accuracy, a thickness measurement is conducted using data from in-vivo obtained from a human subject. This is done as the exact ultrasound velocity in the human body not known. Therefore, it is common to use an average ultrasound velocity $(1540 \mathrm{~m} / \mathrm{s})$ to estimate muscle thickness in medical ultrasound imaging. Hence, the accuracy in thickness measurement evaluated in this experiment is relative.

A healthy male subject (age 25) is recruited to perform a single voluntary isometric contraction on his flexor carpi ulnaris muscle of the right arm. Figure 5.1 shows a measurement setup for the forearm monitoring with the WUS in the pulse-echo mode. The WUS is placed on the surface of the skin along the flexor carpi ulnaris (FCU) muscle with an ultrasound coupling gel. During the experiment, the participant 
is asked to slowly perform voluntary wrist flexion to maximum extension and then return his wrist to the original position. Figure 5.2 shows an example of the raw RF signal acquired during a single voluntary isometric contraction. The estimated tissue thickness is measured using an ultrasound pulse emitted from the WUS, where the thickness is derived from recording the TOF of the ultrasound pulse signal reflected from the bone.

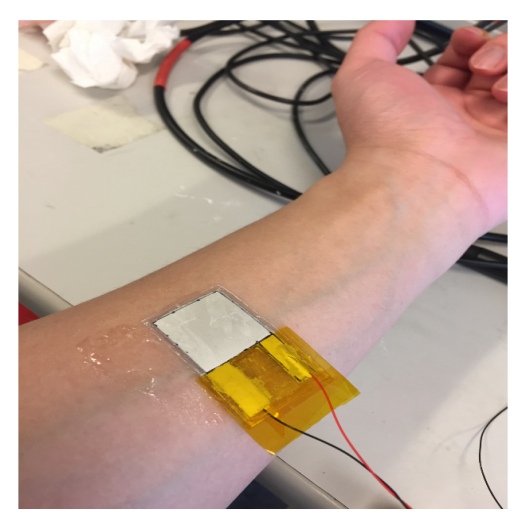

Figure 5.1: In-vivo experiment setup for forearm muscle monitoring using WUS in pulseecho mode.

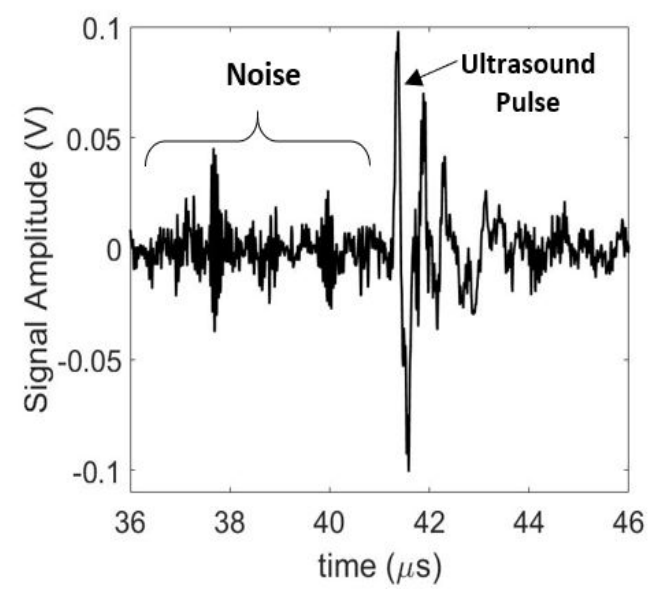

Figure 5.2: Raw RF signal obtained from the forearm monitoring experiment with the indication of ultrasound pulse reflected from the ulnar bone.

Figure 5.3, shows the RF signals after moving averaging with different frame 
numbers. The operation of the moving averaging is discussed in Chapter 3.2. By 3 frames moving averaging, it is able to reduce the effect of the random noise shown in Figure 5.3 (a). With the greater number of the frame averaging, the effect of the noise can be further reduced as shown in Figure 5.3 (a-d).

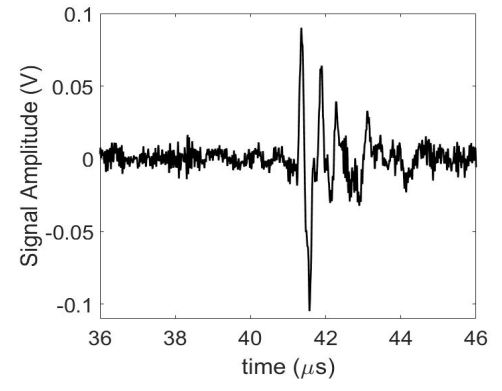

(a)

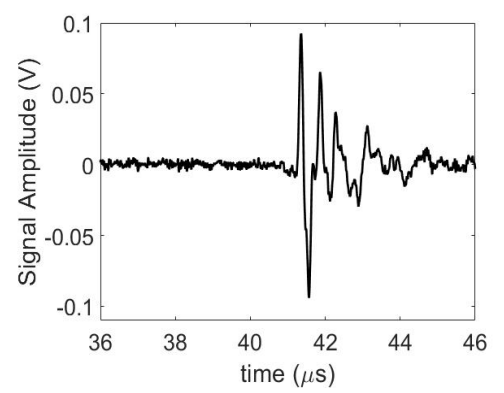

(c)

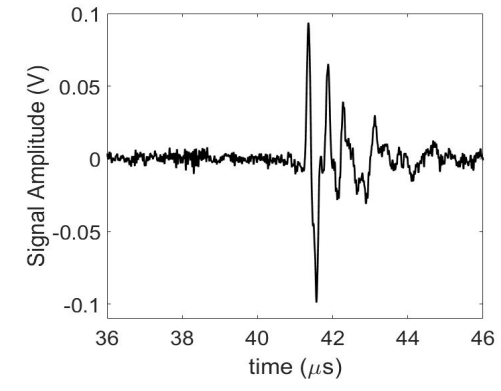

(b)

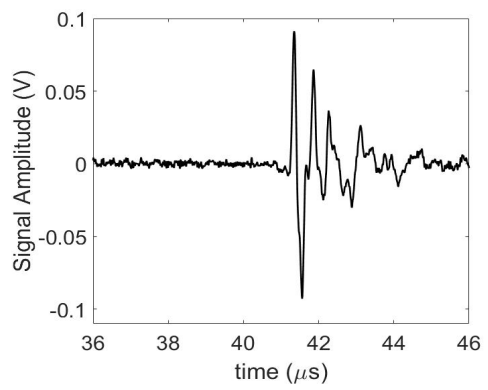

(d)

Figure 5.3: The resultant RF signal after moving averaging with the frame numbers of 3 (a), 9 (b), 17 (c) and 29 (d).

Figure 5.4 shows the SNR (blue cross marks) of the ultrasonic pulse signal with respect to the number of frames in moving averaging. The maximum number of the averaging frames presented in Figure 5.4 is chosen 49 frames due to the fact that there is barely any improvement in the SNR after 45 frames averaging in our experimental setup. One frame averaging corresponds to the raw RF signal (no averaging) in which the SNR is $19.7 \mathrm{~dB}$. The SNR with 45 frames averaging is $36.0 \mathrm{~dB}$. The SNR is calculated by dividing the peak to peak amplitude of the ultrasound pulse of interest $\left(A_{S}\right)$ with twice of the root mean square (RMS) of the noises $(2 \cdot R M S($ Noise $))$ as 
shown in Equation 5.1. Since the random noises are also included in the target raw ultrasonic pulse, the noise free $A_{s}$ value is obtained from the targeted ultrasonic pulse after 49 frames averaging. The $A_{s}$ value with 49 frames averaging is obtained $0.18 \mathrm{~V}$ for the target ultrasonic pulse shown in Figure 5.2. The time range from $36 \mu$ s to 41 $\mu \mathrm{s}$ is selected to calculate the RMS of the noises.

$$
S N R=20 \log _{10}\left(\frac{A_{S}}{2 \cdot R M S(\text { Noise })}\right)
$$

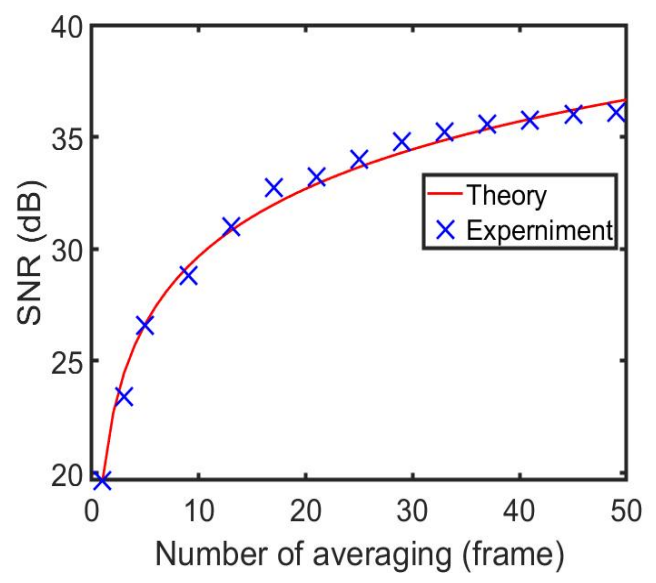

Figure 5.4: The SNR of the RF ultrasound pulse in Figure 5.2 with respect to the frame numbers of moving averaging. The SNR in experiment and in theory are shown by the blue cross marks and the red curve, respectively.

In theory, the improvement of the SNR can be predicted by using Equation 5.2 [108]. Where $\mathrm{SNR}_{0}$ represents the $\mathrm{SNR}$ obtain from the raw RF signal, $\mathrm{SNR}_{1}$ represents the SNR after the moving averaging and $a$ represents the number of the moving averaging frames. As shown in Figure 5.4 by the solid red line, the experimental SNR result has similar trend and good agreement with the theory. The main noise in the WUS measurement system is the random electrical noise in our experimental setup. Therefore, moving averaging can reduce such noises effectively as shown in Figure 
5.3. In theory 5.2, if the average number increase 4 times, SNR becomes 2 times (which is $6 \mathrm{~dB}$ increase). In Figure 5.4, when the number of average increases from 9 to 41 ( $\approx 4$ times), the experiments SNR increases from 28.81 to $35.57 \mathrm{~dB}$. Both the experiments and theory SNR have increased $\approx 6.7 \mathrm{~dB}$.

$$
S N R_{1}=S N R_{0} \cdot \sqrt{a}
$$

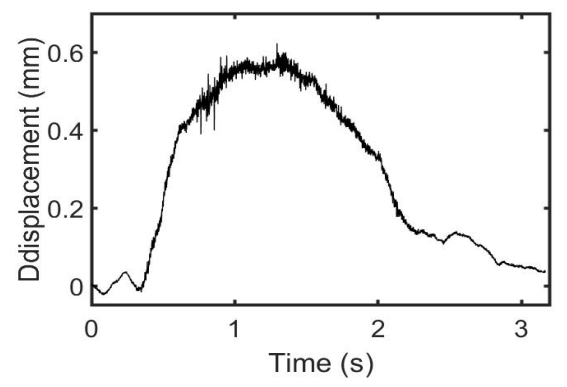

(a)

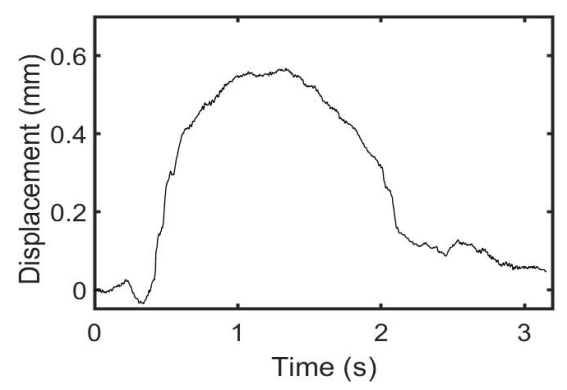

(c)

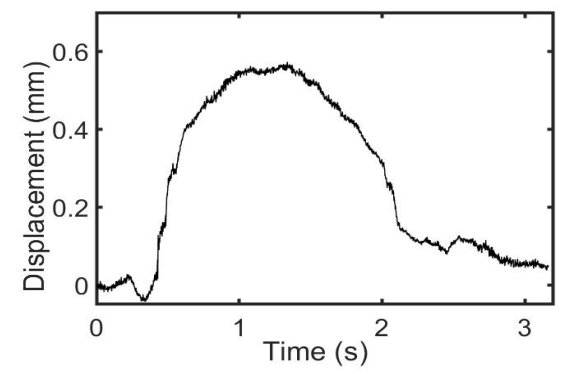

(b)

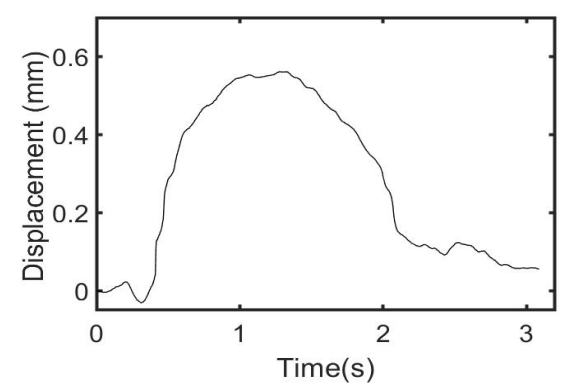

(d)

Figure 5.5: The resultant $R F$ signal after moving averaging with the window size of raw (a), $3(b), 9(c)$ and 45 (d) $\mathrm{ms}$.

After moving averaging on the acquired RF signals, the phase tracking method is applied to each one of them in order to obtain the tissue thickness changes measurement due to the FCU muscle contraction. Figure 5.5 shows the thickness changes obtained from the raw RF signals (a) and the moving averages of 3 frames (b), 9 frames (c), and 45 frames of the RF signals (d). The RMSE is used to evaluate the 
estimation error of the tissue thickness changes with respect to the SNR obtained from the RF signals after the moving averaging. The thickness changes obtained from 45 frames averaging of RF signals is used as a reference for the RMSE calculation. Figure 5.6 shows the result of the RMSE with respect to the number of the moving averaging frames. The result indicates that the RMSE decreased with the numbers of averaging frames. The RMSE is $0.82 \mu \mathrm{m}$ for the moving averaging of 41 frames. Figure 5.7 shows the relationship between the SNR and the RMSE under our experimental conditions obtained using the results in Figures 5.4 and 5.6. This result indicates that as the SNR increases, the RMSE decreases. The following invivo experiments have taken into account with the SNR and RMSE relationship to ensure the RMSE is acceptable to our application and time resolution to capture the detailed changes of the tissue thickness.

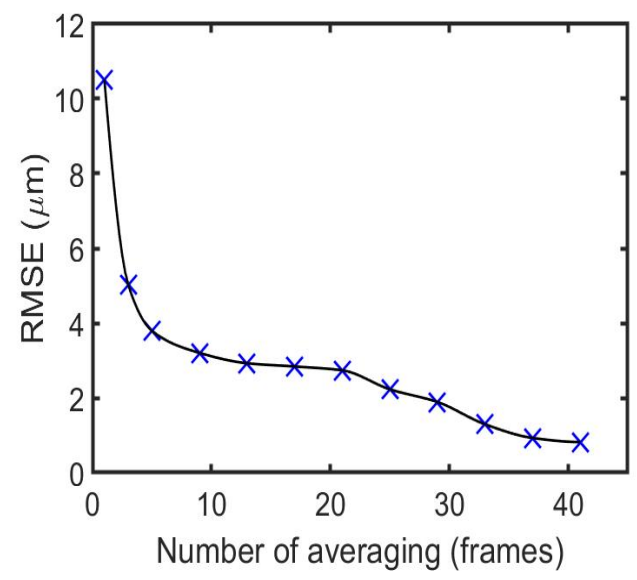

Figure 5.6: The relationship between RMSE and the various window sizes (frames).

However, the moving averaging might lead to the smoothing of the tissue motion captured in the raw ultrasound signals. In order to capture more detailed muscle motion information, the signal acquisition frame rate must be higher. Therefore, the number of averaging must be carefully selected to ensure the quality of the signal 


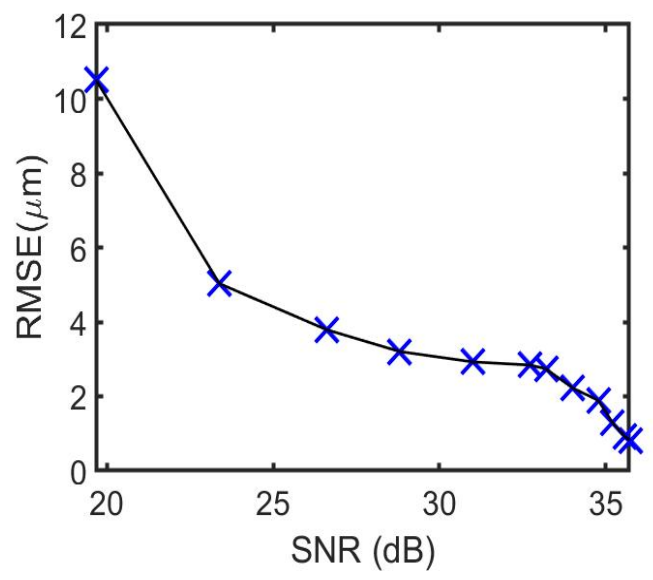

Figure 5.7: The relationship between SNR and the RMSE.

and reduce motion smoothing. The minimum frames averaging filter is used in the following experiments to prevent tissue motion smoothing errors. For in-vivo experiments described in the section 5.3, the greatest frequency of the electromyostimulation (EMS) employed is $30 \mathrm{~Hz}$. Thus, the moving averaging of 17 frames (17 ms) which corresponds to the frame rate of $\approx 60 \mathrm{~Hz}$ is selected since it is almost two times higher than $30 \mathrm{~Hz}$. From the results in Figures 5.4 and 5.6, it can be seen that the SNR and RMSE at 17 frames averaging are $32.74 \mathrm{~dB}$ and $2.84 \mu \mathrm{m}$, respectively.

\subsection{Review of Electromyostimulation}

Electromyostimulation (EMS) is applied to the skeletal muscle and activates muscle contraction. Many studies indicate the benefits of EMS in improving neuron signal synchronization $[109,110]$ and muscle contraction improvement $[111,112]$. EMS can be applied non-invasively using skin surface electrodes. The EMS is applied to a muscle of interest by placing positive and negative electrodes on the skin along the muscle. While the electrical current passes from the positive electrode to the negative electrode, the muscle is stimulated and then contracts. There are many applications in 
EMS including muscle performance improvements for the sports industry [113-115] and muscle rehabilitation $[1,116]$. EMS is also used in muscle tension relaxation and physical therapy [117-119]. EMS applies a stable and periodic electrical current to the muscle by choosing the appropriate pulse waveform, pulse intensity, pulse rate and pulse duration. An example of two consecutive EMS waveforms with the indications of EMS parameters are shown in Figure 5.8. Some EMS devices are able to output different waveform patterns such as square, sinusoidal wave and peaked wave [109]. In addition, EMS waveforms such as monophasic and biphasic waveform also affect the effectiveness in muscle contraction stimulation. Figure 5.9 shows an example of the monophasic and the biphasic EMS waveforms. The study conducted by Laufer,et al. indicates that a biphasic EMS waveform, in which the current is delivered bidirectionally, stimulates the muscle to generate a greater force with a lowered risk of experiencing fatigue compared with other waveforms [120].

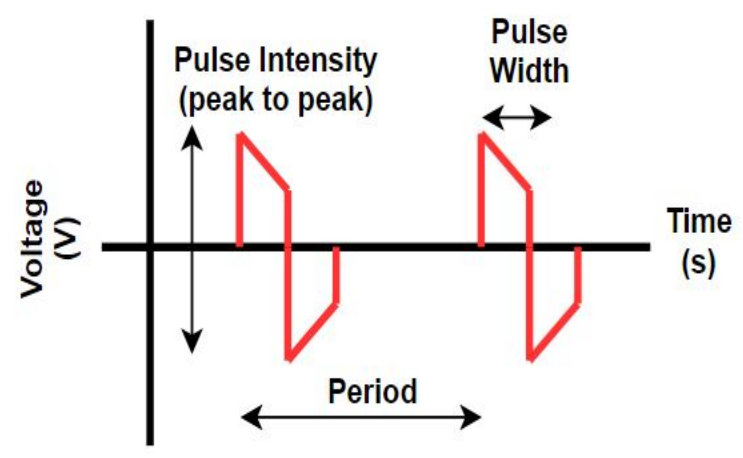

Figure 5.8: An example of the EMS pulse waveform.

The pulse width is the time span in a single EMS pulse where the EMS amplitude is above $0 \mathrm{~V}$ (shown in Figure 5.8). Thus, the greater the pulse width, the more muscle fibers are recruited, resulting in the stronger contraction of the surrounding muscle [109]. Although the overall muscle strength is greater, the muscle gets tired 


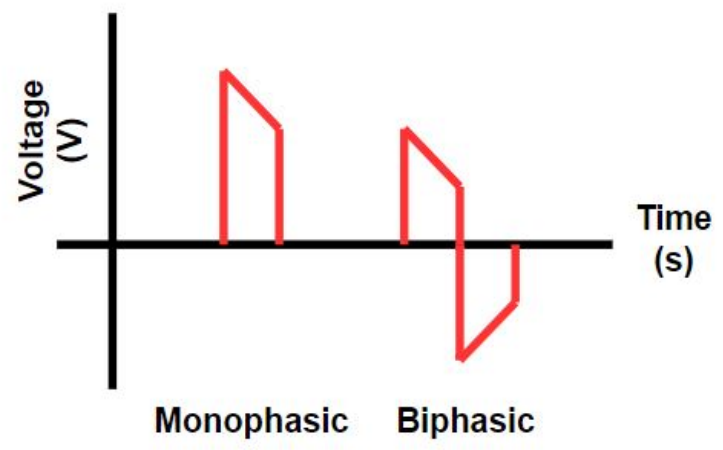

Figure 5.9: An example of the monophasic (left) and biphasic (right) EMS pulse waveforms.

more easily and the user may feel some discomfort. Some studies suggested that the fatigue index is lower with a shorter EMS pulse width $(10 \mu \mathrm{s}$ to $50 \mu \mathrm{s})$. Furthermore, only the selective muscles can be activated by the EMS and the surrounding muscle will not be activated [121]. Therefore, in order to monitor a specific muscle tissue, including the target muscle of this research, the short pulse width from $10 \mu$ s to 50 $\mu \mathrm{s}$ is appropriate. Pulse intensity (amplitude), which is measured in peak to peak voltage, is another parameter that the EMS device is able to control in the pulse waveform (Figure 5.8). The higher the EMS intensity, the stronger depolarization on the neuron underneath the electrodes in which more motor fibers are activated. Muscle density and muscle fiber composition may change the electrical resistivity in muscle contraction. Thus, a low pulse intensity may not be able to activate the muscle contraction due to the resistivity of the tissue. In addition, muscle fatigue also occurs quicker and more regularly in high intensity EMS [109,118].

The EMS pulse repetition frequency is the number of electrical pulses fired within a second. As the pulse frequency increases, the number of muscle twitches per second also increases. The period between two consecutive EMS pulses are calculated by 1 divided by the pulse frequency (Figure 5.8). In a low EMS pulse frequency, the 
muscle can contract and then relax fully, which is called a single muscle twitch. Since a synchronized contraction can be evoked in a low frequency EMS, some contraction properties can be observed [122], such as the maximum contraction distance, contraction time, relaxation time, half relaxation time and contraction velocity. These parameters can provide the valuable information on the behavior of the muscle for the use of the sports industry and rehabilitation. In addition, low frequency EMS reduce the chance of muscle fatigue and avoid subject discomfort. By monitoring the muscle behavior in different EMS frequencies, we can observe the single twitch muscle contraction, unfused tetanus contraction and fused tetanus contraction. These muscle behaviors provide crucial information on muscle fiber types compositions [3, 49], muscle fatigue assessment $[2,40]$ and muscle recovery $[1,116,123]$. The EMS is used during muscle monitoring in tensiomyography (TMG) to allow the muscle monitoring in a reliable and repeatable manner [8].

\subsection{Experimental Configuration in Muscle Moni- toring with Electromyostimulation}

In the following in-vivo experiments, EMS is used to evoke the triceps brachii lateral head muscle (TBLH) of three healthy male subjects. The purpose of this experiment is to achieve muscle contractile parameters through muscle monitoring with the WUS measurement system. The muscle contraction is evoked by the EMS in various EMS pulse repetition frequencies. This research is approved by the Carleton University Research Ethics Committee. All three subjects from age 23 to 25 have consented to participate in the experiment and agreed to provide the information in any publications about the results or conclusions from the study. Table 5.1 provides the health condition information about the subjects. 
Table 5.1: The health conditions of the subjects.

\begin{tabular}{|c|c|c|c|}
\hline Subjects & $\# 1$ & $\# 2$ & $\# 3$ \\
\hline Age & 25 & 24 & 23 \\
\hline $\begin{array}{c}30 \text { mins strength } \\
\text { exercise per week } \\
\text { (such as weight lifting) }\end{array}$ & 0 & 1 & 3 \\
\hline $\begin{array}{c}\text { Records of } \\
\text { muscle disease }\end{array}$ & No & No & No \\
\hline $\begin{array}{c}\text { Muscle pain } \\
\text { before the experiment }\end{array}$ & No & No & No \\
\hline $\begin{array}{c}\text { Muscle fatigue } \\
\text { before the experiment }\end{array}$ & No & No & No \\
\hline $\begin{array}{c}\text { Sickness } \\
\text { before the experiment }\end{array}$ & No & No & No \\
\hline
\end{tabular}

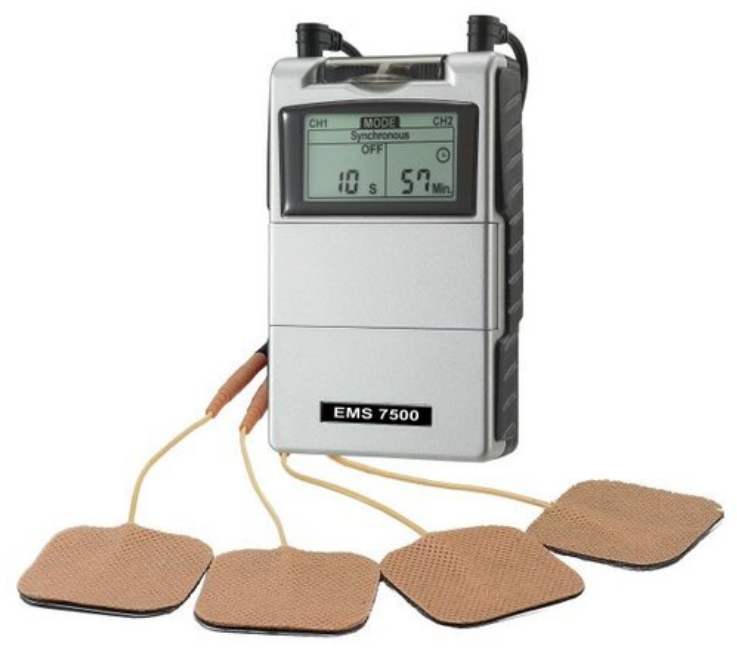

(a)

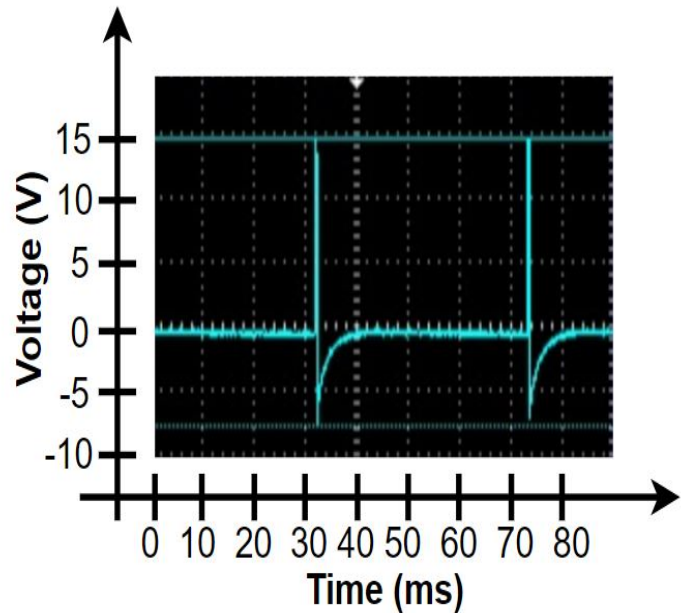

(b)

Figure 5.10: EMS 7500 device is shown on the left and an example EMS pulse waveform is shown on the right.

Table 5.2: EMS 7500 output voltage in various power levels measured with 500 ohms resistance.

\begin{tabular}{|c|c|c|c|c|c|c|c|c|}
\hline Power level & 1 & 2 & 3 & 4 & 5 & 6 & 7 & 8 \\
\hline Voltage (V) & 0 & 0 & 1 & 7 & 21 & 31 & 42 & 50 \\
\hline
\end{tabular}


The EMS device used in the experiment is the EMS 7500 model which is shown in Figure 5.10 (a). The EMS device evokes the muscle contraction by applying an electric current through the skin surface using the surface electrodes. The biphasic rectangular waveform sent by the EMS device is shown in Figure 5.10 (b). The EMS pulse width, pulse intensity and pulse time period are $0.3 \mathrm{~ms}, 21 \mathrm{~V}$ and $41.6 \mathrm{~ms}$, respectively. The EMS 7500 is able to provide the voltage from 0 to $50 \mathrm{~V}$ with 500 ohms resistance by selecting the power level from 1 to 8 . Figure 5.11 shows the configuration circuit in measuring the voltage of the EMS device across a $500 \mathrm{ohm}$ resistor. Where the +ve and -ve are representing the positive and negative conductive wires of the EMS device, respectively. Table 5.2 shows the voltage measured for the EMS device in different power levels using 500 ohms resistance. Various EMS frequencies are applied to the muscle to observe the response of the TBLH muscle from low to high frequency stimuli $(2 \mathrm{~Hz}$ to $30 \mathrm{~Hz})$. To avoid activation of the other muscles, pulse width of $50 \mu \mathrm{s}$ is selected. Due to the resistivities of the soft tissues (including the skin and muscles) are different among the subjects, the voltage required to evoke muscle contraction is different from person to person. In addition, to avoid muscle fatigue and subject discomfort, the minimum power level that evokes muscle contraction is selected for each subject. The qualitative details of the EMS waveform parameters are shown in Table 5.3.

Table 5.3: The experimental setup for the EMS device in each subject.

\begin{tabular}{|c|c|c|c|}
\hline EMS parameters & Subject 1 & Subject 2 & Subject 3 \\
\hline Pulse width & $50 \mu \mathrm{s}$ & $50 \mu \mathrm{s}$ & $50 \mu \mathrm{s}$ \\
\hline Pulse power level & 4 & 4.5 & 5 \\
\hline $\begin{array}{c}\text { Pulse intensity (voltage) } \\
\text { measured by Figure } 5.11\end{array}$ & $7 \mathrm{~V}$ & $15 \mathrm{~V}$ & $21 \mathrm{~V}$ \\
\hline Pulse rate & 2 to $30 \mathrm{~Hz}$ & 2 to $30 \mathrm{~Hz}$ & 2 to $30 \mathrm{~Hz}$ \\
\hline
\end{tabular}




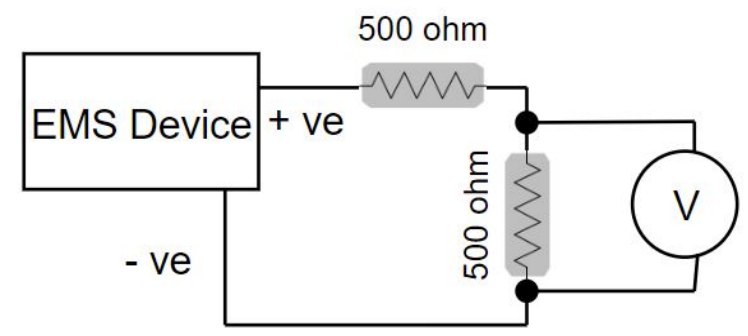

Figure 5.11: The configuration circuit in measuring the EMS voltage across a 500 ohm resistor.

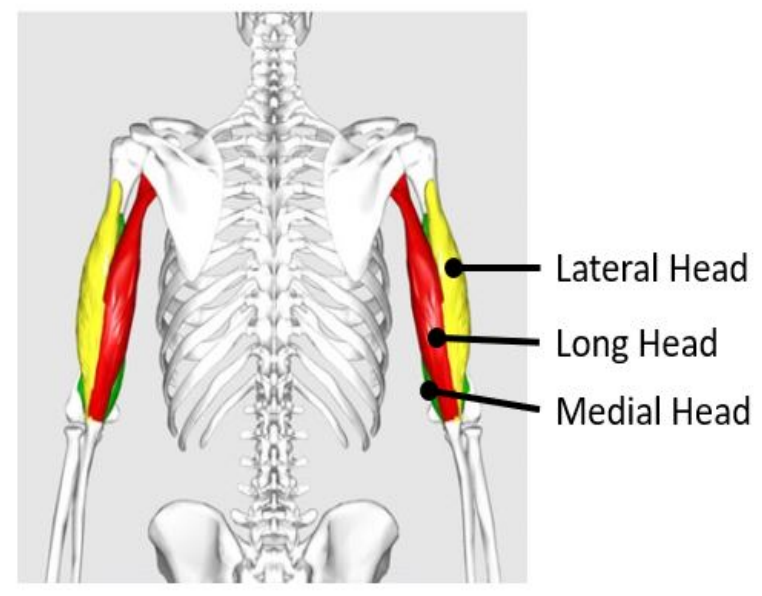

Figure 5.12: The triceps muscle antonym. Image is reproduced from "File:Triceps brachii muscle06.png" by Anatomography and the image is licensed under Creative Commons BY-SA $2.1 \mathrm{jp}$ license. Image is free access online at https://commons.wikimedia.org/wiki/File:Triceps_brachii_muscle06.png [126].

The triceps brachii lateral head is located below the deltoid muscle. The deltoid muscle is located on the uppermost part of the arm and the shoulder [124]. The lateral triceps muscle rises from the dorsal surface of the humerus in the lateral view and extends to the region of the lateral intermuscular septum [125]. Figure 5.12 shows the anatomy of the upper arm muscles and the location of the lateral triceps muscle [126]. The muscle highlighted in yellow, red, and green are the triceps brachii lateral head (TBLH), long head, and medial head, respectively. Compared to the 
medial head, the TBLH is easier to find since it is closer to the surface of the skin. Furthermore, it is more visible while contracting the upper arm muscle. Therefore, to locate the TBLH muscle visually, the subjects are asked to stretch their upper arm before the experiment. To stimulate the TBLH muscle, a $40 \mathrm{~mm} \mathrm{X} 40 \mathrm{~mm}$ electrodes (Lemonbest, Hong Kong) are used to apply the electric current to the muscle. The TBLH muscle monitoring configuration is shown in Figure 5.13. The first EMS electrode, which is connected to the red positive wire, is placed on the bottom of the deltoid muscle and overlapped with the TBLH muscle. The second electrode, which is connected to the black negative wire, is placed below the first electrode and towards the lateral side of the upper arm. The distance between the electrodes is approximately $60 \mathrm{~mm}$ to limit the muscle contraction activity in the upper arm. The WUS is placed between the two electrodes as seen in Figure 5.13. An ultrasound coupling gel is applied between the WUS and the surface of the skin. To ensure the stability of the WUS, gaffer tape is applied on top of the protection layer of the WUS and the copper wires. The tape is not applied on the sensing area of the WUS to ensure no additional force is applied to the muscle during the experiment.

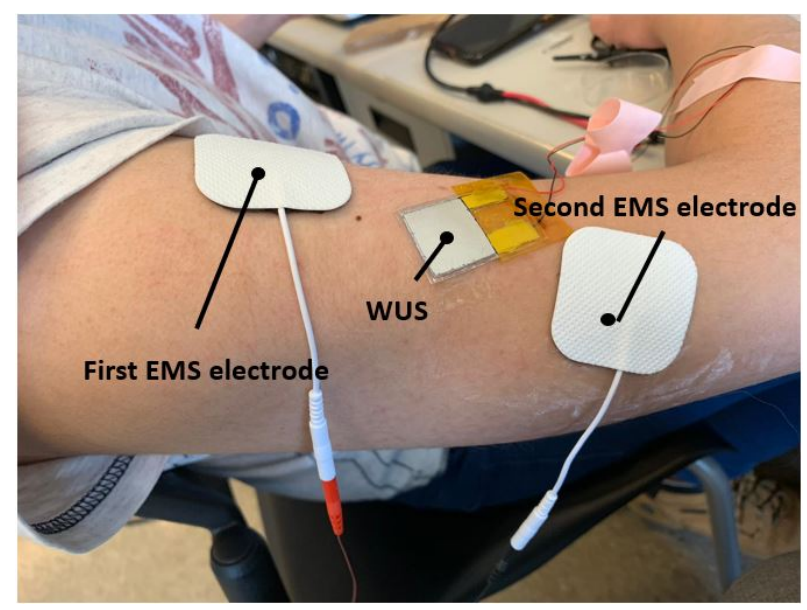

Figure 5.13: Photograph of WUS and EMS electrodes attached to the skin surface of the arm for monitoring tetanus contraction of TBLH in pulse-echo mode. 


\subsection{Experimental Result}

The ultrasound RF signals are recorded during the muscle contraction evoked by the EMS by using the WUS in the pulse echo method in M-mode. The frame rate of the WUS system is $1 \mathrm{kHz}$. For data pre-processing, moving averaging is performed to increase the SNR of the RF signal. Since the highest EMS pulse repetition frequency applied to the muscle is $30 \mathrm{~Hz}$ which is equivalent to electrical pulse in every $\approx 33$ $\mathrm{ms}$, to minimize the effect of the motion smoothing, the 17 frames (17 ms) of moving averaging is applied to all the RF signals. As discussed in section 5.1, the RMSE and average SNR are $2.84 \mu \mathrm{m}$ and $32.74 \mathrm{~dB}$, respectively, for the 17 frames moving averaging. The averaged RF signal is then applied to quadrature demodulation method to find the phase of the RF signals. Then, the phase tracking method is used to convert the phase difference of the RF signals between the two consecutive frames into the tissue displacement. Figure 5.14 shows the changes in TBLH muscle thickness for Subject 1 due to the muscle contraction evoked by the EMS at different pulse repetition frequencies $(2$ to $30 \mathrm{~Hz}$ ). The results for subjects 2 and 3 are presented in the Appendix.

In the tissue displacement observed at $2 \mathrm{~Hz}$, the TBLH muscle is able to fully relax between each EMS pulse as seen in Figure 5.14 (a). When the EMS pulse frequency increases to $6 \mathrm{~Hz}$ and above, partial relaxation between two pulses are observed up to $16 \mathrm{~Hz}$. In addition, the relaxation time of the TBLH muscle is reduced by increasing the EMS frequency. As the pulse rate increases, the muscle relaxation time decreases, and the maximum tissue thickness changes gradually increases. This phenomenon is called as wave summation in muscle contraction [38]. After a rapid increase in muscle thickness, the partial relaxation is observed, which is known as unfused tetanus contraction [18]. As the EMS frequency increases, the muscle contraction fluctuation 


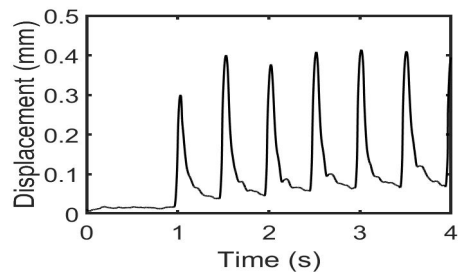

(a) $2 \mathrm{~Hz}$

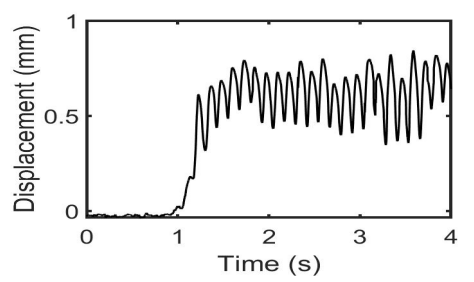

(d) $8 \mathrm{~Hz}$

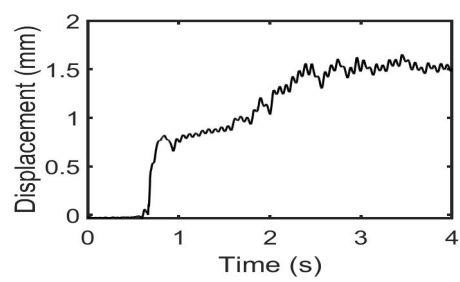

(g) $14 \mathrm{~Hz}$

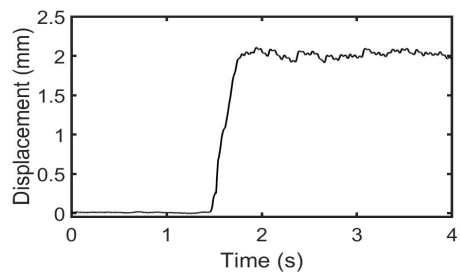

(j) $20 \mathrm{~Hz}$

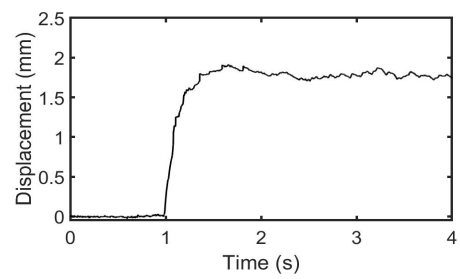

(m) $26 H z$

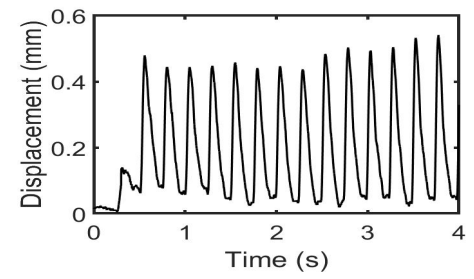

(b) $4 \mathrm{~Hz}$

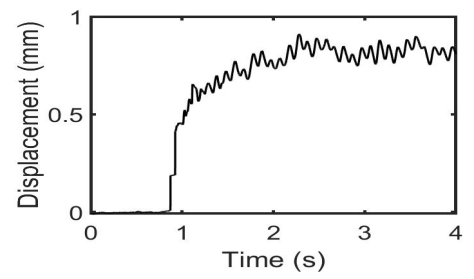

(e) $10 \mathrm{~Hz}$

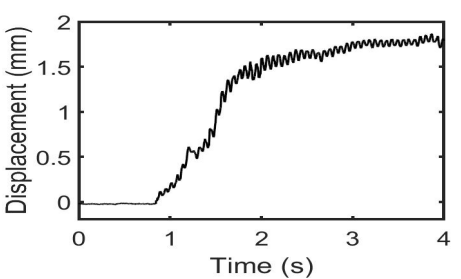

(h) $16 \mathrm{~Hz}$

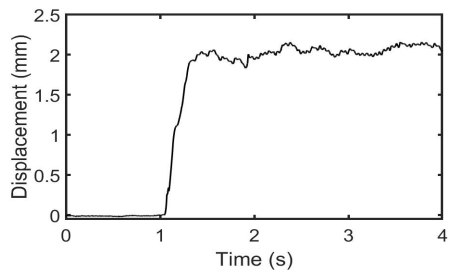

(k) $22 \mathrm{~Hz}$

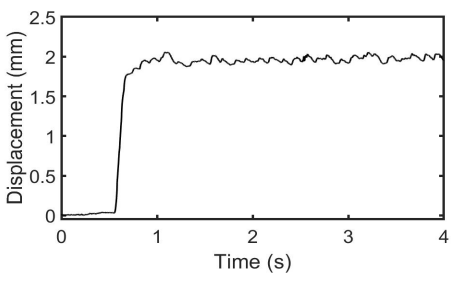

(n) $28 H z$

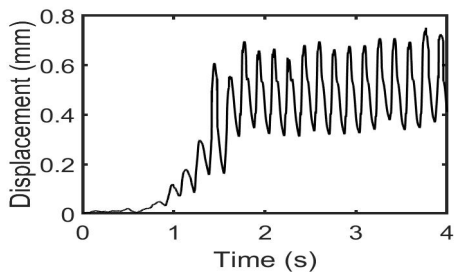

(c) $6 \mathrm{~Hz}$

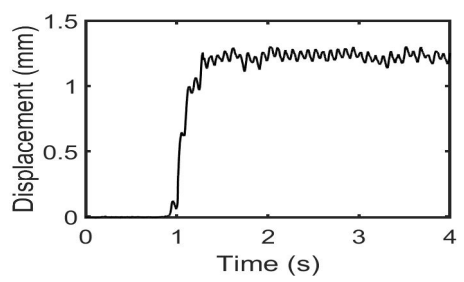

(f) $12 \mathrm{~Hz}$

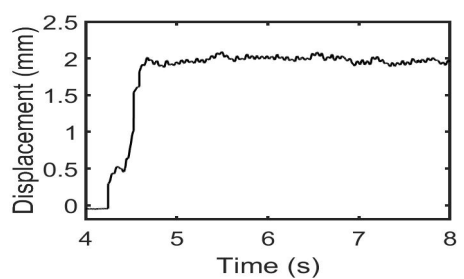

(i) $18 \mathrm{~Hz}$

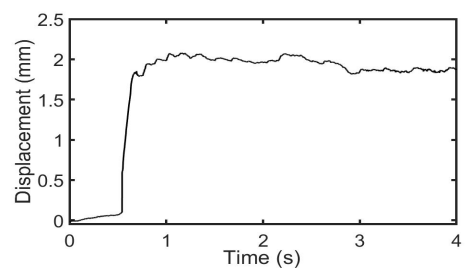

(l) $24 \mathrm{~Hz}$

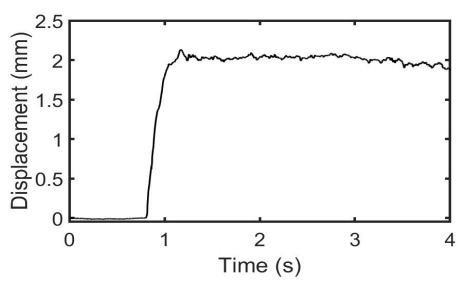

(o) $30 H z$

Figure 5.14: Subject 1 TBLH tissue displacement due to muscle contraction evoked by EMS (from pulse repetition frequency $2 \mathrm{~Hz}$ to $30 \mathrm{~Hz}$ ). 
become smaller. Fused tetanus of the muscle contraction is observed at the EMS frequency greater than $20 \mathrm{~Hz}$ when each muscle twitch is unable to differentiate from one another, where the muscle twitches are completely fused. The muscle can no longer relax and further contract at the fused tetanus. In general, when the pulse frequency increase, the tissue displacements at the local minimum and local maximum increases as observed in Figure 5.14 (d)-(h). At the frequency of $24 \mathrm{~Hz}$ and above, tetanic contraction is achieved, and the muscle twitches are fused completely in Figure 5.14 (1)-(o). This result matches with the motor recruitment theory which describes that the central nervous system controls the muscle strength and contraction length by increasing the frequency of the stimulus $[48,52]$. Occasionally, some tissue motions

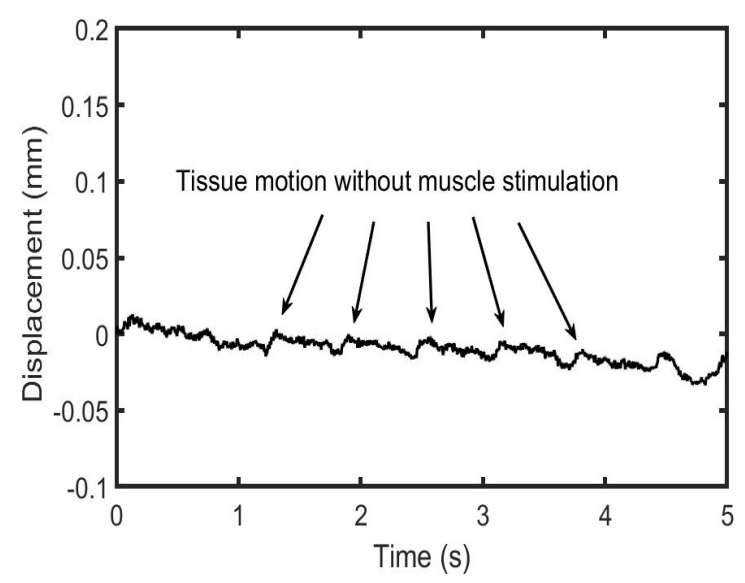

Figure 5.15: Muscle motion observed without any electrical stimulation.

are observed even without any EMS as shown in Figure 5.15. These periodic motions may be caused by arterial blood pressure. Hence, the WUS may also be able to detect the heart rate of the subject during muscle monitoring. The observed displacement due these tissue motions are relatively small (around $10 \mu \mathrm{m}$ ) when comparing to the muscle twitch $(<300 \mu \mathrm{m})$. Therefore, we did not account the errors caused by these motion in this research. In the future, some adjustments might require to remove the 
effect of such motions from the resultant muscle displacement.

Table 5.4: Average initial thickness of the triceps brachii lateral head muscle for the three subjects.

\begin{tabular}{|c|c|c|c|}
\hline & Subject 1 & Subject 2 & Subject 3 \\
\hline Muscle Initial Thickness & $21.56 \pm 0.47 \mathrm{~mm}$ & $29.09 \pm 0.30 \mathrm{~mm}$ & $30.16 \pm 0.37 \mathrm{~mm}$ \\
\hline
\end{tabular}

To obtain the total tissue thickness without the EMS, the peak detection technique is used to find the TOF of the ultrasound echo from the bone and the result of the tissue thickness calculation using Equation 3.2. The estimated TBLH thickness for each subject is shown in Table 5.4. Subject 3 has the largest TBLH muscle thickness among the three subjects. As the thickness of the tissue increase, the resistivity to electrical stimuli also increase [127]. Thus, the required minimum pulse intensity is $21 \mathrm{~V}$ to evoke the TBLH muscle contraction for Subject 3. However, there are other factors which may have an effect on the muscle electrical resistivity, including the tissue composition, the surface skin moisture, body temperature, gender, distance between the electrodes and neuron reaction $[128,129]$.

\subsection{Discussion}

\subsubsection{Muscle Contractile Property}

Several muscle contractile properties are extracted from the muscle displacement analysis of EMS frequency at $2 \mathrm{~Hz}$. An example of the contractile properties are shown in Figure 5.16. These contractile parameters such as maximum contraction displacement (Th), contraction time (Tc, contraction velocity (Vc), $90 \%$ relaxation time $\left(\mathrm{RT}_{90} \%\right)$ and $10 \%$ relaxation time $\left(\mathrm{RT}_{10} \%\right)[8,25,44,123]$. A horizontal baseline is set for each muscle twitch where the baseline intersects at the start of the muscle contraction. The 
baseline, which is colored in blue, is shown in Figure 5.16. Th is the maximum muscle displacement from a single stimulation. Hence, Th is the displacement between the baseline and the peak of the muscle displacement. Tc is the time it takes for the muscle to contract to the maximum twitch. RT is time it takes for the muscle to fully relax. $\mathrm{RT}_{10 \%}$ and $\mathrm{RT}_{90 \%}$ are the time duration in which the displacement becomes $10 \%$ and $90 \%$ of the peak muscle displacement, respectively. Table 5.5 shows the contractile parameters extracted from the TBLH muscle contraction evoked at $2 \mathrm{~Hz}$ EMS frequency for each subject. For each subject, there are 8 muscle twitches in total that are examined, and Table 5.5 also shows the average and standard deviation of the contractile properties.

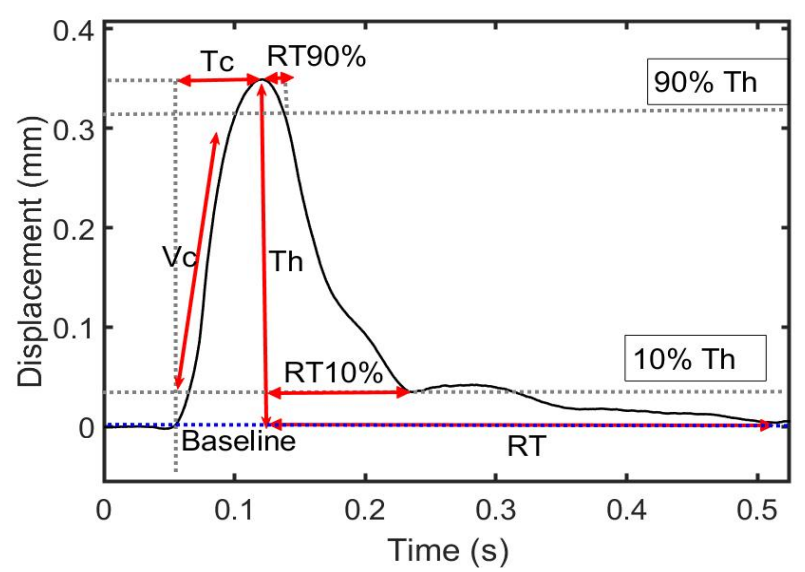

Figure 5.16: Tissue displacement observed and muscle contractile properties extracted from a single twitch due to muscle contraction evoked by EMS at $2 \mathrm{~Hz}$.

\subsubsection{Fusion Index}

Fusion index (FI) is a useful indicator for muscle tetanus progression [39,44,130,131]. In addition, the FI indicates the ratio of the muscle relaxation during the electrical stimulation in a particular frequency. The FI at a chosen EMS frequency is estimated 
Table 5.5: The contractile properties extracted from tissue displacement evoked by 2 $\mathrm{Hz}$ EMS frequency.

\begin{tabular}{|c|c|c|c|}
\hline Subjects & $\# 1$ & $\# 2$ & $\# 3$ \\
\hline Tc $(\mathrm{ms})$ & $70 \pm 2$ & $77 \pm 1$ & $78 \pm 1$ \\
\hline Th $(\mathrm{mm})$ & $0.385 \pm 0.037$ & $0.737 \pm 0.107$ & $0.474 \pm 0.0582$ \\
\hline $\mathrm{RT}_{90 \%}(\mathrm{~ms})$ & $17 \pm 2$ & $16 \pm 4$ & $27 \pm 5$ \\
\hline $\mathrm{RT}_{10 \%}(\mathrm{~ms})$ & $160 \pm 35$ & $126 \pm 18$ & $216 \pm 47$ \\
\hline $\mathrm{Vc}(\mathrm{mm} / \mathrm{s})$ & $5.503 \pm 0.618$ & $9.570 \pm 1.408$ & $6.105 \pm 0.736$ \\
\hline
\end{tabular}

by: $F I=(a / b) X 100$, where 'a' and 'b' are the displacement at the local minimums and maximums, respectively, as shown in Figure 5.17. We can calculate the FI from

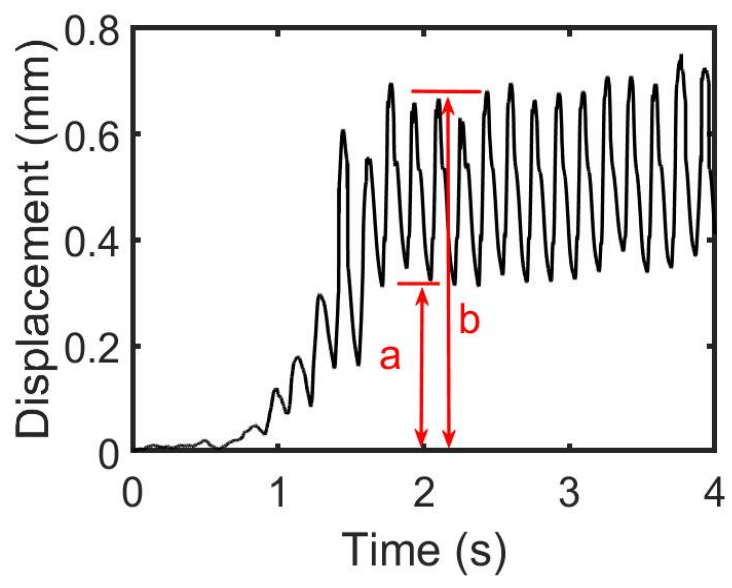

Figure 5.17: An example of fusion index calculation.

each muscle contraction and then calculate the average FI for the corresponding EMS frequency. By estimating the FI at multiple frequencies, a FI-frequency curve (FFC) can be obtained as a function of the EMS frequency [39]. Figure 5.18 presents the FFC obtained for each subject. The FFC has an 'S' shape and a sigmoid function is used for the curve fitting. The sigmoid equation with three parameters $(\mathrm{q}, \mathrm{r}, \mathrm{s})$ are defined in Equation 5.3, where q,r, and $s$ are the parameters in the curve fitting and 
$f$ is the EMS frequency.

$$
F I(f)=\frac{q}{1+e^{-r(f-s)}}
$$

The FFC is an important indicator in evaluating the rehabilitation of the muscle from

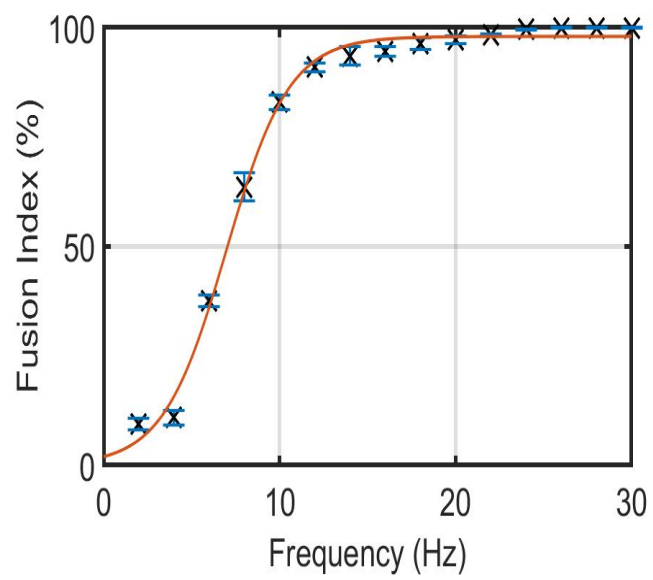

(a) Subject 1

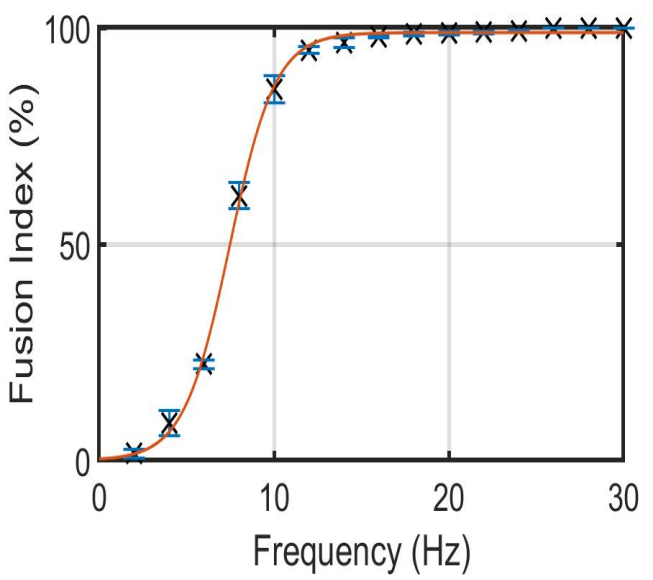

(b) Subject 2

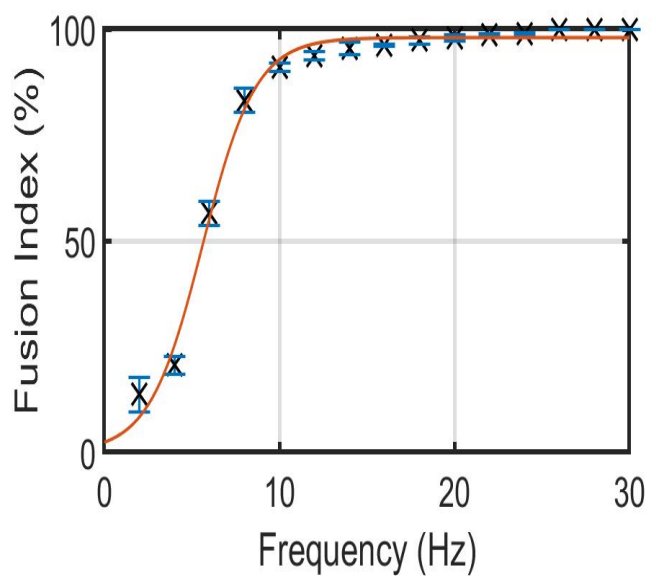

(c) Subject 3

Figure 5.18: The fusion index frequency curve (FFC) obtained from each subject.

an injury and the effectiveness of the physical training [132]. It is also able to evaluate the muscle fiber type and the fiber composition by finding the frequency which evokes both unfused and fused tetanus contraction [39]. The minimum unfused and fused frequencies are estimated at the $10 \%$ and $90 \%$ of the FI in the FFC, respectively. 
Some challenges are associated in predicting fusion frequency from FFC since it requires long experiment times with multiple frequencies. Furthermore, muscle fatigue may occur in high EMS frequencies and the subject may also experience discomfort and possibly pain due to the electrical pulses. A recent study performed muscle monitoring evoked by EMS on rats using a force sensor [44]. In their experiment, the rats' muscles are surgically opened to place a force sensor directly onto the muscle of interest while stimulating the muscle by EMS. They proposed using the contractile properties (Tc and $\mathrm{RT}_{10 \%}$ ) extracted from a single twitch obtained at a low EMS frequency to predict the unfused and fused tetanus frequencies of the muscle of interest [44]. Due to the fact that the unfused and fused tetanus frequencies are highly related to the contraction time and relaxation time, they found a strong correlation between these two tetanus frequencies, which are both estimated via the FFC and the contractile properties obtained from a force measurement of a single twitch at a single low EMS frequency. By adapting the method proposed by Watanabe, et al., we can also predict the unfused and fused tetanus frequencies by computing the contractile properties obtained from a single muscle displacement evoked at $2 \mathrm{~Hz}$ EMS frequency by using the Equation 5.4 and Equation 5.5. The Equation 5.4 is used to calculate the fused tetanus frequency and Equation 5.5 is used to calculate the fused tetanus frequency.

$$
\begin{aligned}
& F_{1}=\frac{1}{\left(C T+R T_{10 \%}\right)} \\
& F_{2}=\frac{1}{\left(C T+R T_{90 \%}\right)}
\end{aligned}
$$

Table 5.6 shows the estimated fusion frequency from the FFC and the contractile properties obtained from the muscle displacement of a single twitch evoked at $2 \mathrm{~Hz}$ EMS frequency. Figure 5.19 presents the relationships of unfused/fused frequencies 
Table 5.6: Comparison of the unfused and fused frequencies estimated from the FFC and from contractile parameters of a single twitch at $2 \mathrm{~Hz}$ EMS frequency.

\begin{tabular}{|c|c|c|c|}
\hline Subjects & $\# 1$ & $\# 2$ & $\# 3$ \\
\hline $\begin{array}{c}\text { Estimated minimum stimulation frequency } \\
\text { for unfused tetanus } \\
\text { obtained from FFC }\end{array}$ & $4.60 \mathrm{~Hz}$ & $3.26 \mathrm{~Hz}$ & $2.31 \mathrm{~Hz}$ \\
$\begin{array}{c}\text { for unfused tetanus } \\
\text { Estimated minimum stimulation frequency }\end{array}$ & $\begin{array}{c}4.82 \\
\text { predicted from a 2 Hz muscle displacement }\end{array}$ & $\begin{array}{c}3.77 \\
\pm 0.84 \mathrm{~Hz}\end{array}$ & $\begin{array}{c}2.37 \mathrm{~Hz} \\
\pm 0.41 \mathrm{~Hz}\end{array}$ \\
\hline $\begin{array}{c}\text { Estimated Fusion Frequency } \\
\text { obtained from FFC }\end{array}$ & $11.32 \mathrm{~Hz}$ & $10.51 \mathrm{~Hz}$ & $9.31 \mathrm{~Hz}$ \\
\hline $\begin{array}{c}\text { Estimated Fusion Frequency } \\
\text { predicted from a 2 Hz muscle displacement }\end{array}$ & $\begin{array}{c}11.46 \\
\pm 0.34 \mathrm{~Hz}\end{array}$ & $\begin{array}{c}10.65 \\
\pm 0.37 \mathrm{~Hz}\end{array}$ & $\begin{array}{c}9.43 \\
\pm 0.38 \mathrm{~Hz}\end{array}$ \\
\hline
\end{tabular}

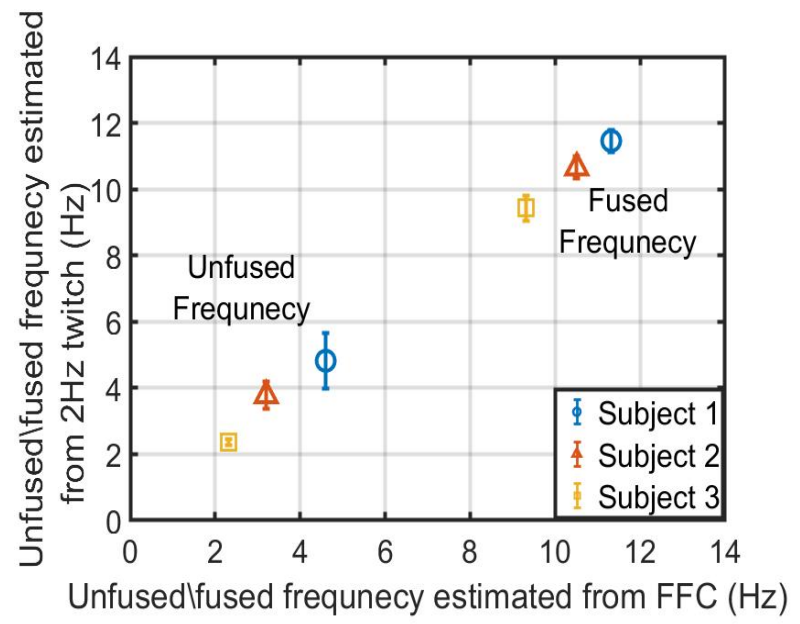

Figure 5.19: The relationships of unfused and fused frequencies obtained from the FFC and from contractile parameters of a single twitch at $2 \mathrm{~Hz}$ EMS frequency.

obtained by the two methods. From this result, we observed that the estimated unfused/fused frequencies from the FFC have a good agreement with the prediction of a $2 \mathrm{~Hz}$ muscle displacement. The lowest fusion frequency $(\approx 9.5 \mathrm{~Hz})$ is obtained from Subject 3, which may indicate that his TBLH muscle has a higher slow muscle fiber composition than the other two subjects [39]. In addition, as shown in Table 
5.6 , it took a longer time $(78 \mathrm{~ms})$ for his TBLH muscle to contract to the maximum displacement which also indicates the increase of the ratio in slow muscle fiber in his TBLH muscle $[38,39]$.

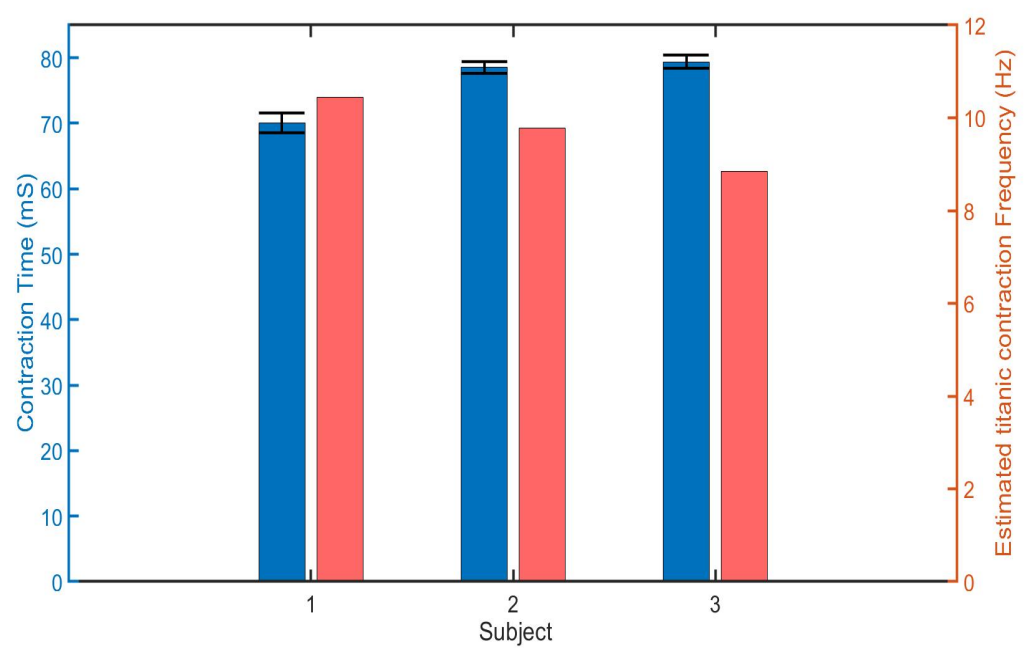

Figure 5.20: The relationship between the contraction time and fusion frequency of the TBLH predicted from the FFC.

Figure 5.20 shows a bar chart representing the relationship of the fusion frequency and the contraction time of the TBLH muscle observed from the three human subjects. The blue color bar represent the average contraction time obtained at $2 \mathrm{~Hz}$ EMS frequency and the red color bar represent the fused tetanus contraction estimated from the FFC. The high contraction time and low fused tetanus frequency observed for Subject 3 TBLH muscle may be a result of the high slow muscle composition in his triceps. Several studies have indicated that with different exercises and muscle training, the muscle compositions may change, such as from fast muscle fiber to slow muscle fiber and vise versa $[133,134]$. Additionally, regular strengthening exercise is able to increase the muscle mass [45]. Compared to subjects 1 and 2, Subject 3 performs regular weightlifting exercise training which may cause the increase in slow muscle within the upper arm muscle including the triceps $[45,135]$. 


\section{Chapter 6}

\section{Conclusion and Future Studies}

\subsection{Conclusions}

This thesis aims to perform muscle thickness monitoring through ultrasound in pulse echo mode, in order to monitor skeletal muscle contractile properties. Due to the limitations of the handheld conventional ultrasonic probe (bulky, rigid and heavy), it is difficult to achieve muscle monitoring without restricting muscle movement. Inconsistent probe movement may induce measurement error (motion artifacts). A wearable ultrasonic sensor (WUS), which is lightweight, thin and flexible, has been developed by our research group. As it can adhere directly onto the surface of the skin, it can reduce such motion artifacts caused by the conventional probe.

The thesis began with providing the background information on skeletal muscle anatomy, physiology of skeletal muscle contraction, ultrasound physics and the current technologies in muscles study. Then, this thesis continued with the selection of piezoelectric materials for the WUS and the thickness measurement techniques.

The experiments on the phantom were performed to determine the reliability and accuracy of the WUS thickness measurements. In the first phantom experiment, 
the thickness changes estimated by three signal processing techniques were compared with the measurement results by the laser displacement sensor (LDS). Since the phase tracking method provided the best accuracy, it is used throughout the thesis. In the second phantom experiment, a mechanical simulation system was designed and constructed to evaluate the repeatability and accuracy of the WUS thickness measurements. It was found that the repeatability of the WUS thickness measurements for a stable object was $+/-1 \mu \mathrm{m}$ and the accuracy was $1.79 \mu \mathrm{m}$. Then, the maximum absolute error of the WUS thickness measurements on dynamic movement in comparison with the LDS was found to be $7.2 \mu \mathrm{m}$. In addition, the WUS was able to measure thickness changes of $3 \mu \mathrm{m}$.

Then, muscle monitoring on human volunteers were conducted using the WUS. Since human tissues are more complex than the controlled phantom environment, there is more signal attenuation of the ultrasound echo strength. Hence, moving averaging was performed to the acquired RF ultrasound signal to suppress the noise for improving the signal-to-noise ratio (SNR). To further investigate the effect of moving averaging on the SNR and the thickness measurement accuracy, the number of the averaging of frames (from 3 frames to 45 frames) were performed on the RF signals, which were acquired from the forearm during the voluntary muscle contraction measurement. It was observed that the increased moving averaging frame numbers increased the SNR of the desired echo. Furthermore, the increase in SNR also decreased the error in thickness measurement. For the in-vivo experiment, 17 frames moving averaging was performed to the acquired RF signal. 17 frames averaging provided the relative accuracy of $2.84 \mu \mathrm{m}$ and the SNR of $32.74 \mathrm{~dB}$. After that, three volunteers were recruited to monitor their triceps brachii lateral head (TBL) muscle by the WUS measurement system. To generate involuntary muscle contractions, the TBL muscle was evoked by an electromyostimulation (EMS) device in various EMS pulse 
repetition frequencies (from $2 \mathrm{~Hz}$ to $30 \mathrm{~Hz}$ ). The WUS measurement system acquired the ultrasound RF signals during the muscle contractions. Then, the quadrature demodulation and phase tracking method were applied to the averaged RF signal to calculate the tissue thickness. The muscle contraction properties such as contraction time, relaxation time and contraction velocity were obtained from a single twitch of the muscle contraction evoked by the EMS at $2 \mathrm{~Hz}$. Furthermore, the tetanic progression represented by fusion index (FI) was calculated from the thickness measurement evoked at each EMS frequency. By estimating the FI at multiple frequencies, a FIfrequency curve (FFC) was obtained as a function of the EMS frequency. The FFC is an important indicator in evaluating the rehabilitation of the muscle from an injury and the effectiveness of the physical training [132]. It is also able to evaluate the muscle fiber type and the fiber composition by finding the frequency which evokes unfused and fused tetanus contractions [44]. The minimum unfused and fused tetanus frequencies are defined at the $10 \%$ and $90 \%$, respectively, of the FI in the FFC.

However, some challenges are associated in predicting fusion frequency from FFC since the prediction requires long experiment times with multiple frequencies. Furthermore, muscle fatigue may occur in high EMS frequencies and the subject may also experience discomfort from the applied EMS pulses. This thesis adapted the method suggested by Watanabe, et al. of using a single thickness changes measurement evoked at low EMS frequencies to estimate the unfused and fused frequencies. This study analyzed and compared the unfused and fused tetanus frequencies obtained from the two methods, and it was found that there was a good agreement between them. 


\subsection{Future Study}

There are still many improvements that can be made to the WUS measurement system, measurement methods and signal processing techniques. Some suggestions for future developments are listed below.

In this thesis, the accuracy of several thickness estimation methods were evaluated. The phase tracking methods provided the best accuracy under our experimental design. But there are still other signal processing methods, such as Hilbert transform, which may improve accuracy with minimum computational power to the WUS measurement system. Qiu, et al. suggested that a Hilbert transform based quadrature demodulation can achieve a fast and reliable ultrasonic measurement [136]. In additional, moving averaging is a common technique in improving SNR. However, motion smoothing may filter out some unique behavior of the muscle, which may be relevant to the study. The limitation and the selection of the frames of averaging needs to be further investigated. In addition, other DSP techniques (such as adaptive filter) can be used to filter RF signals and improve the SNR.

In the phantom experimental design, a mechanical simulation experiment is performed to evaluate the accuracy and repeatability of the WUS measurement system. Occasionally, the thickness changes of the phantom are not consistent. This is due to the dissipation of the applied force through the sides of the phantom, where the PMMA plate is absent. To improve the consistency of this experiment, a PMMA plate could be placed on each side of the phantom (except the top) to ensure the thickness changes are localized at the center of the phantom. This improved phantom experiment configuration can be used with multiple ultrasound sensors attached in all dimensions.

In the accuracy evaluation, several specifications of the WUS measurement system 
are yet to be studied. A unique feature of the WUS is its flexibility and ability to conform to the curvature of the tissue adequately. However, the accuracy of the WUS is yet to be confirmed due to the sensor curvature. I suggest making several phantoms with varying degrees of curvature and compare the thickness measurement results with the LDS. Furthermore, the motion artifacts caused by the hand held conventional ultrasonic probe can be investigated by comparing it with the LDS. Additionally, the measurement error due to angular misalignment of the WUS is not discussed in this thesis. This measurement error is caused by the reflection of the ultrasound pulses from the bone, which are not fully received by the sensor due to the angular changes as shown in Figure 7 in the Appendix. Since muscle thickness increases during contraction and the angular misalignment may occur during the orientation of the WUS, it is no longer parallel with the bone. Further research on the effect of the WUS misalignment can improve the monitoring of muscle contraction. The effect of angular misalignment can be studied using a linear 3-axis goniometer stage. When the transnational stage changes the angle, the data can be acquired for accuracy evaluation.

In the in-vivo experiment, several EMS parameters were selected including the pulse intensity, pulse width and pulse repetition frequency. To reduce subject discomfort due to EMS, the low pulse width of $50 \mathrm{~ms}$ and the minimum pulse intensity were selected. However, these EMS parameters may directly change the muscle response. The extracted contraction parameters such as contraction time and contraction intensity may also change. Therefore, further investigation is required on the effect of these EMS parameters towards the extracted contractile parameters. Additionally, unfused/fused tetanus frequencies can be used to evaluate the rehabilitation and effectiveness of the treatment from an injury. The muscle fiber composition can also be revealed from these contraction parameters. More subjects are needed to be tested in 
order to verify the findings in predicting unfused and fused tetanus frequencies from a single thickness measurement evoked by 2 Hz EMS frequency. Furthermore, the relationship between the muscle fiber composition and unfused/fused tetanus frequencies can be studied further. 
Appendices 


\section{Appendix A}

\section{A.1 Quadrature Demodulation}

Fourier theory indicates that a signal can be decomposed into multiple sinusoidal signals with different amplitude, frequency and phase [85]. Hence, the ultrasound signal S(n) can be represented as a sinusoidal function which has the center frequency of the ultrasonic transducer as follows.

$$
S(n)=A(n) \cos w(n) n T+\theta(n)
$$

where, $n$ is the sample index begins from 1 to the total number of samples in each frame, $\mathrm{A}(\mathrm{n})$ is the amplitude of the received signal, $w=2 * p i * f c * T s$ where $f c$ is the center frequency and $T s$ is the sampling time, $\theta(\mathrm{n})$ is the instantaneous phase of the ultrasonic signal which contains the information about the motion from the object. In the process of demodulation, the RF signal is multiplied by a reference sine and cos wave with a frequency $\mathrm{f}_{d}$.

$$
\begin{gathered}
S R=2 e^{-j w_{d} n T s} \\
S R=2 \sin \left(w_{d} \cdot n \cdot T s\right)+2 j \cdot \cos \left(w_{d} \cdot n \cdot T s\right)
\end{gathered}
$$

where $\mathrm{w}_{d}$ is the angular demodulation with a chosen frequency. The in-phase and quadrature component of the quadrature demodulator are presented as $I(n)$ and $Q(n)$ respectively.

$$
I(n)=L P F\{S(n) \cdot(2 \cos (w o \cdot n \cdot T))\}
$$




$$
Q(n)=L P F\{S(n) \cdot(-2 \sin (w o \cdot n \cdot T))\}
$$

where $L P F$ is equal to low pass filter. The FIR filter is chosen due to its linearity, stability and low computation complexity [10]. The purpose of the low- pass filter in quadrature demodulation limits the passband of the RF signal and reduce the influence of the noise to the result. By using trigonometric identities, I(n) and Q(n) can be described as follows

$$
\begin{gathered}
I(n)=L P F\{[2 A(n) \cos (w \cdot n \cdot T+\theta(n)) \cdot \cos (w o \cdot n \cdot T)\} \\
I(n)=L P F\{A(n) \cos ((w+w o) n T+\theta(n)) \cdot \cos ((w-w o) \cdot n \cdot T+\theta(n)))\} \\
I(n)=A(n) \cos (\Delta w \cdot n \cdot T+\theta(n))
\end{gathered}
$$

and

$$
\begin{gathered}
Q(n)=L P F\{[-2 A(n) \cos (w \cdot n \cdot T+\theta(n)) \cdot \sin (w o \cdot n \cdot T)\} \\
Q(n)=L P F\{A(n) \cos ((w+w o) n T+\theta(n)) \cdot \cos ((w-w o) \cdot n \cdot T+\theta(n)))\} \\
Q(n)=A(n) \sin (\Delta w \cdot n \cdot T+\theta(n))
\end{gathered}
$$

where $\Delta w$ is the downshift of the echo frequency. The complex baseband signal can be described as

$$
J(n)=I(n)+j Q(n)
$$

in which the instantaneous phase can be found in

$$
\angle J(n)=\Delta w(n) \cdot n \cdot T+\theta(n)
$$


One of the main assumptions from the traditional quadrature demodulation is that the echo center frequency is identical to the transmitting frequency [84]. However, due to attenuation the center frequency of the transmitting signal changes over time, therefore a center frequency tracker is needed to continuously monitoring the changes in center frequency. The frequency tracker is based on the assumption of the highest amplitude in the frequency domain of the RF signal is corresponding to the center frequency of the signal. In addition, with a high sampling frequency acquisition system, it is possible to obtain the minor change in the center frequency within $S(n)$. Figure 1 shows the flow chart of the quadrature demodulation.

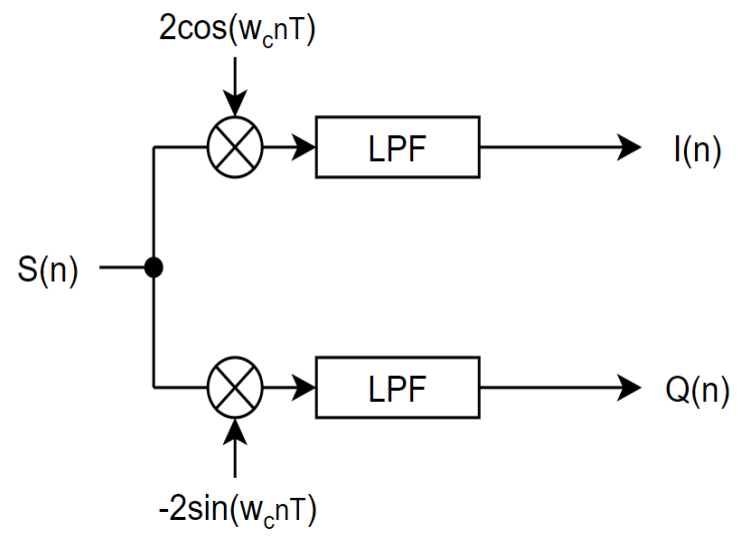

Figure 1: The flow chart of the quadrature demodulation. 


\section{A.2 Laser Displacement Sensor}

A reference thickness measurement is needed for evaluating the accuracy of the WUS. To avoid additional motion artifact which may alter the measurement result, a noncontact measurement is a better choice. The laser displacement sensor is a noncontact method and is selected to use as a reference thickness measurement because of this technology can provide accuracy up to $+/-0.4 \mu \mathrm{m}$ or $0.02 \%$ linearity within the measurement range $[102,137]$. The laser sensor has been used to perform precision thickness measurement $[138,139]$. Some studies on soft tissue thickness changes measurements have used the laser displacement sensor as a golden standard to compare with other techniques in interest [139-141].

The following LDS measurement principles and specifications are based on the LDS (Model: LK-H055K, Keyence, Japan) [102]. The functionality of the laser is begun with emitting a laser beam by a linear laser emitter. The laser beam hit the surface of the object and bounce to the receiver. The non-contact laser displacement sensor detects the object displacement based on the triangulation theory. Since the position of the emitter and the receiver is fixed within the laser displacement sensor, a triangular path is formed as shown in Figure 2. By finding the location of the beam on the laser receiver, the angle between the emitted laser beam and the returned laser beam $(\theta)$ can be measured. By applying trigonometry to the $\theta$ and the distance between the emitter and receiver, the object location can be determined.

Figure 3 shows the reference distance and measurement range of the LDS. The reference distance of the LDS is $46.3 \mathrm{~mm}$ which is corresponding to the optimal sensing distance of the sensor. The accuracy of LDS is related to the distance of the material under measurement. At the optimal measurement range $(+/-5 \mathrm{~mm}$ away from the reference distance), the accuracy is $1 \mu \mathrm{m}$. However, the accuracy 


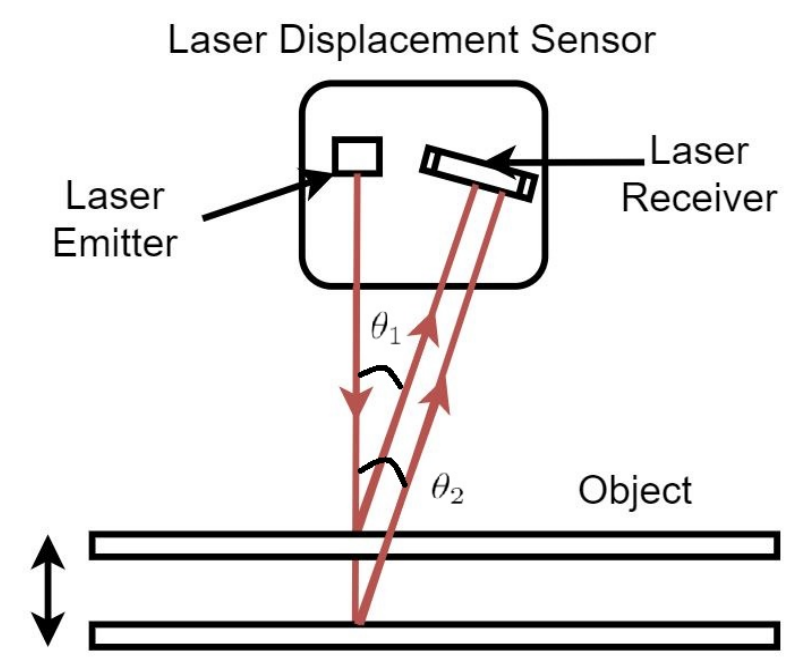

Figure 2: Measurement principle and configuration of the laser displacement sensor.

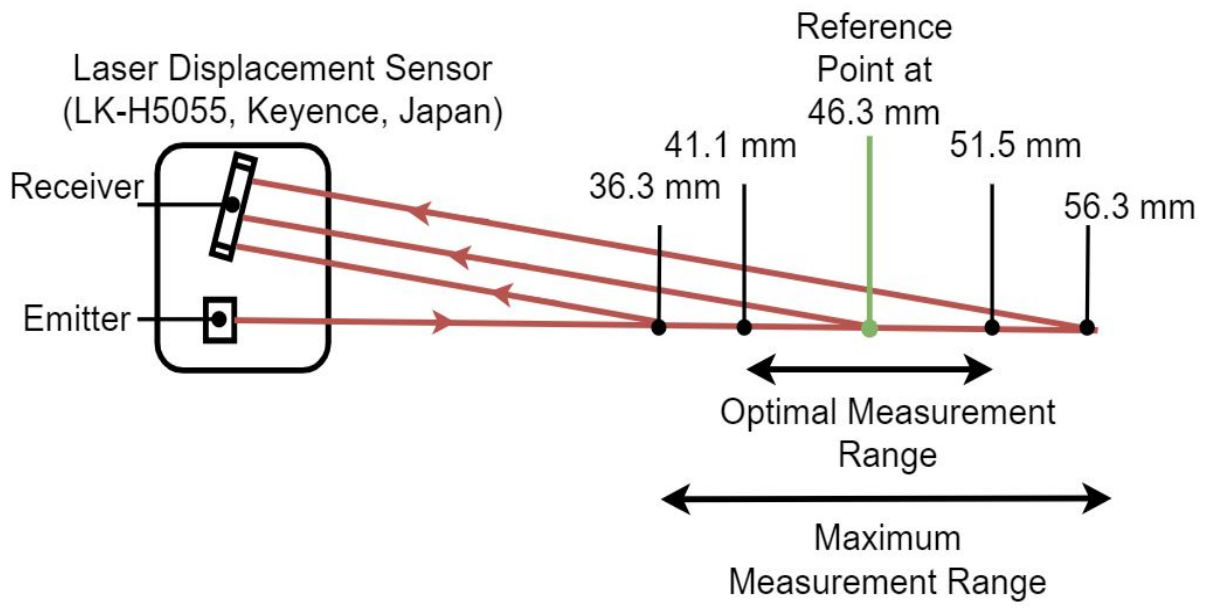

Figure 3: The reference distance and the measurement range of the laser displacement sensor (LK-H055, Keyence, Japan).

decreases to approximately $2 \mu \mathrm{m}$ when the object distance is larger than the optimal measurement range. The resolution of the LDS is $0.025 \mu \mathrm{m}$.

Figure 4 shows the laser waveform obtained from the LDS laser receiver. The $\mathrm{y}$-axis of the plot shows the amplitude of the laser detected on the receiver and the 


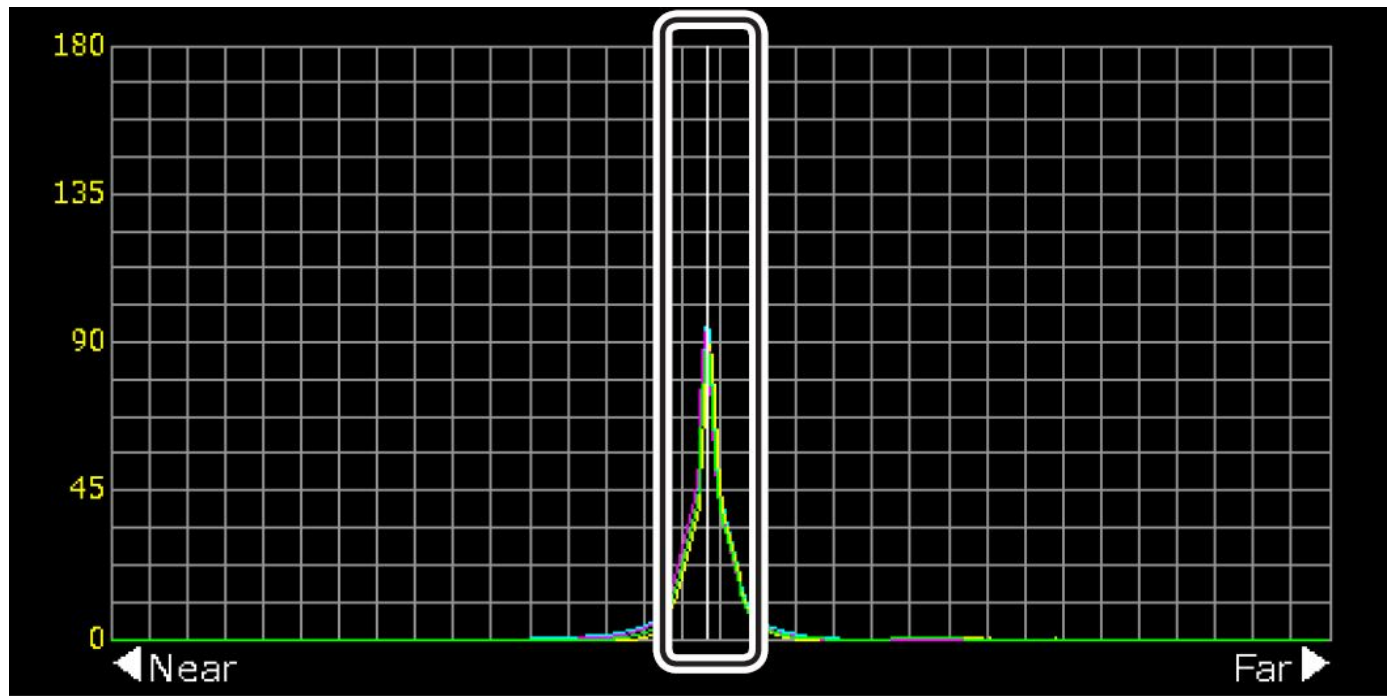

Figure 4: A typical laser waveform obtained from the LDS receiver.

$\mathrm{x}$ - axis shows the location of the laser light hit on the receiver. The middle point at the $\mathrm{x}$ - axis shows the reference distance ( $46.3 \mathrm{~mm}$ away from the LDS). The yellow peak, shows the location of the laser beam detected on the receiver. The near and far on the plot indicates the location of the object respect to the reference point.

\section{A.3 Triceps Brachii Lateral Head Muscle Monitoring}

The following diagrams shows the changes in TBLH muscle thickness for Subject 2 and 3 due to the muscle contraction evoked by the EMS at different pulse repetition frequencies (2 to $18 \mathrm{~Hz})$. 


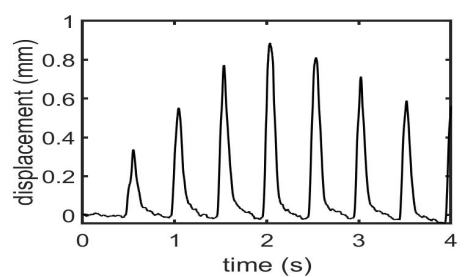

(a) $2 H z$

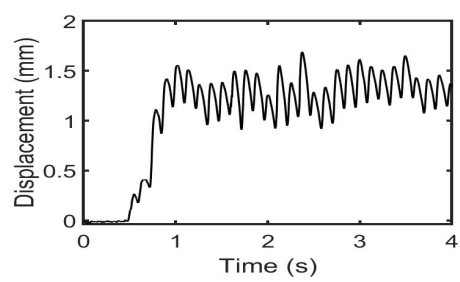

(d) $8 \mathrm{~Hz}$

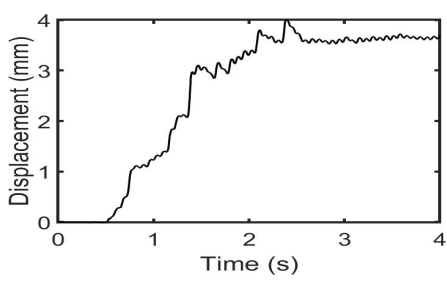

(g) $14 \mathrm{~Hz}$

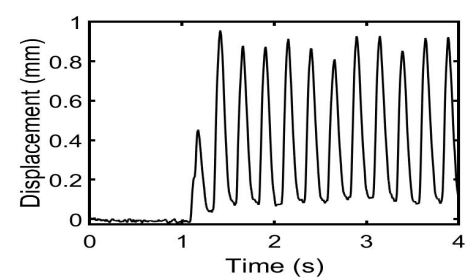

(b) $4 \mathrm{~Hz}$

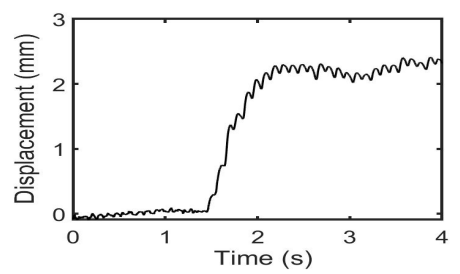

(e) $10 \mathrm{~Hz}$

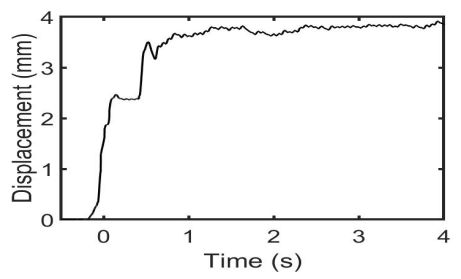

(h) $16 \mathrm{~Hz}$

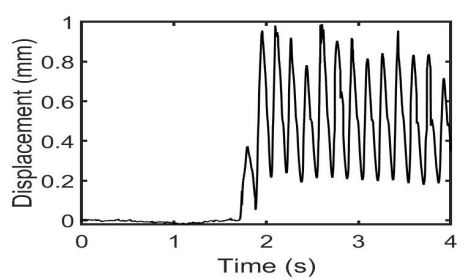

(c) $6 \mathrm{~Hz}$

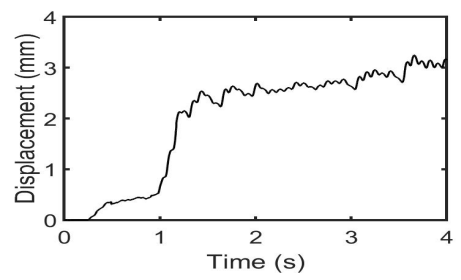

(f) $12 \mathrm{~Hz}$

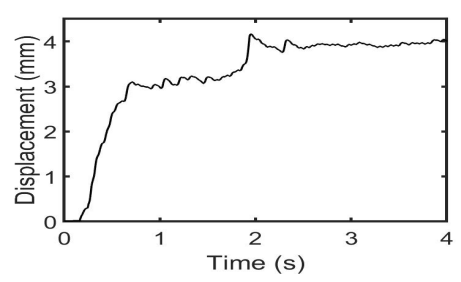

(i) $18 \mathrm{~Hz}$

Figure 5: Subject 2 TBLH tissue displacement due to muscle contraction evoked by EMS (from pulse repetition frequency $2 \mathrm{~Hz}$ to $18 \mathrm{~Hz}$ ) 


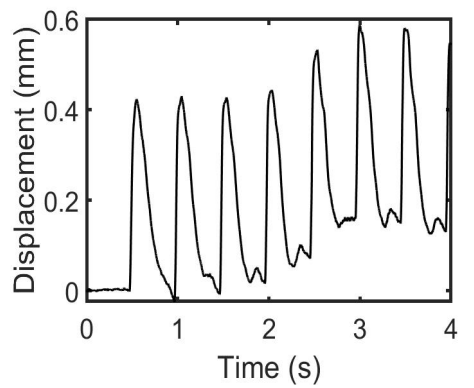

(a) $2 \mathrm{~Hz}$

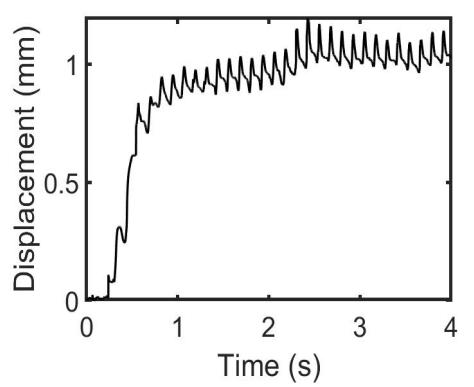

(d) $8 \mathrm{~Hz}$

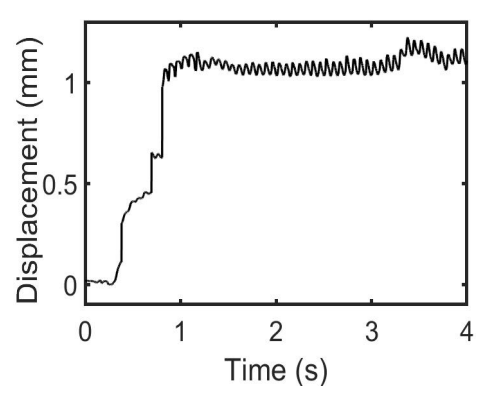

(g) $14 \mathrm{~Hz}$

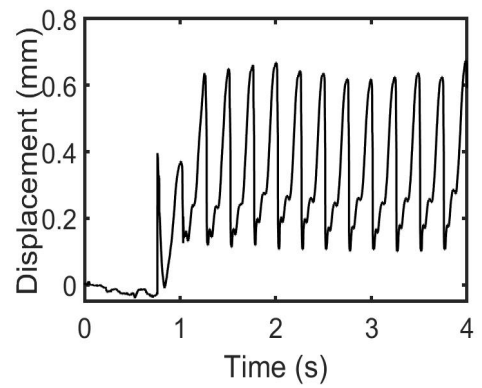

(b) $4 \mathrm{~Hz}$

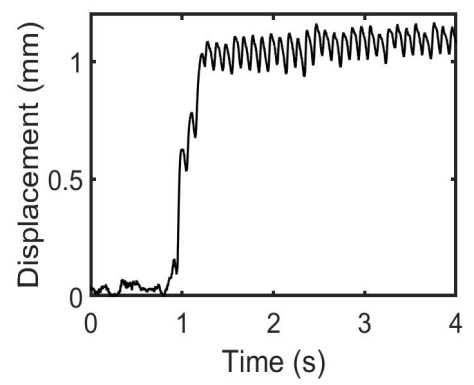

(e) $10 \mathrm{~Hz}$

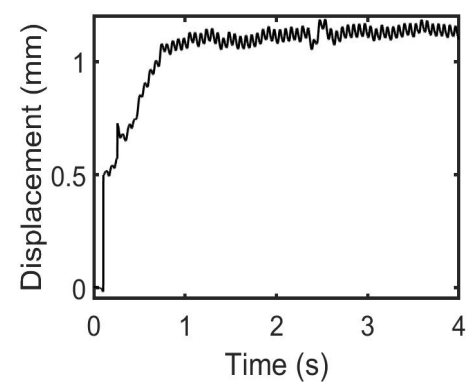

(h) $16 \mathrm{~Hz}$

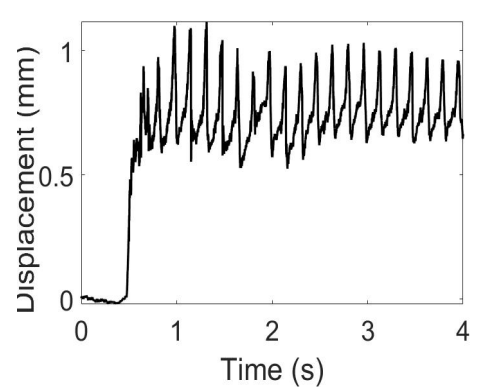

(c) $6 \mathrm{~Hz}$

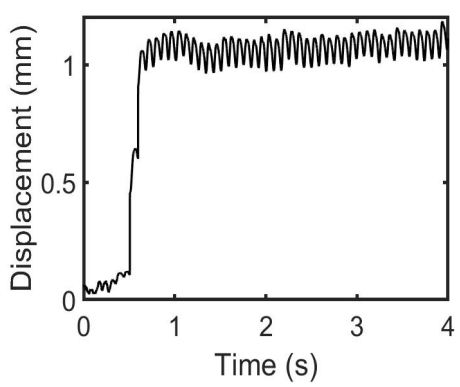

(f) $12 \mathrm{~Hz}$

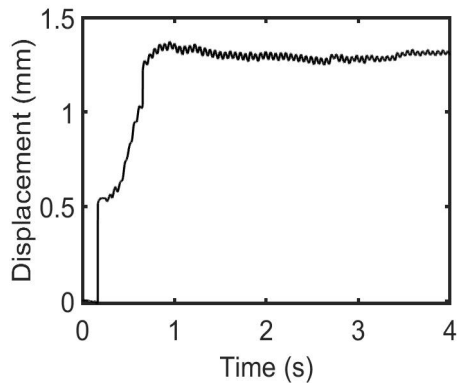

(i) $18 \mathrm{~Hz}$

Figure 6: Subject 3 TBLH tissue displacement due to muscle contraction evoked by EMS (from pulse repetition frequency $2 \mathrm{~Hz}$ to $18 \mathrm{~Hz}$ ).

\section{A.4 Angular Misalignment}

In the measurement model, the ultrasonic beam travels perpendicular to the transducer and the reflected ultrasound returns back to the transducer perpendicularly, as shown in Figure 7. Thus, the ultrasonic transducer should be kept in parallel with the 
layers of tissues, in order to obtain the maximum reflection of ultrasonic signal. The signal strength is reduced as the difference in angular misalignment increase between the transducer and tissues, resulting in lower signal-to-noise ratio [142]. It is not yet discovered of the measurement error caused by the angular misalignment. However, in the dynamic measurement of tissue displacement during muscle contraction, the ultrasound may experience an angular misalignment with respect to the tissue boundary surface.
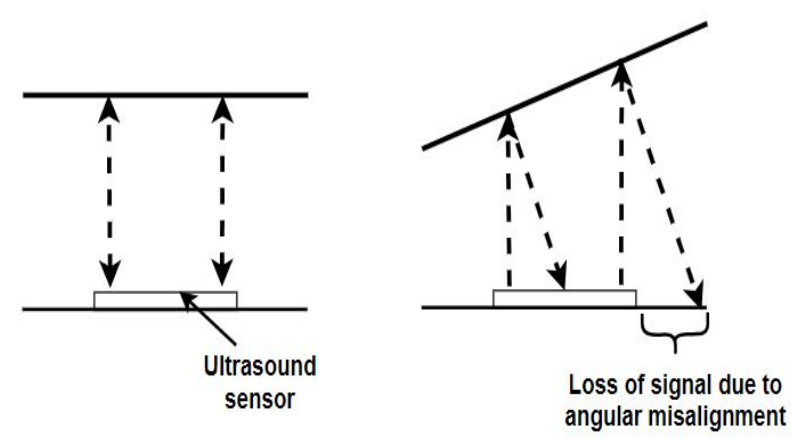

Figure 7: Example of an angular misalignment between the ultrasonic sensor and the boundary of a medium.

\section{A.5 Figures Permission}

The following image demonstrate the permission granted email sent by IOP Publishing to use the figures from "Estimate the minimum stimulation frequency necessary to evoke tetanic progression based on muscle twitch parameters" by S. Watanabe, et al. 
Dear Elliot Yeung,

Thank you for your request to reproduce material published by IOP Publishing in your thesis, "A Method to Characterize Skeletal Muscle Contractile Properties Using a Wearable Ultrasonic Sensor".

Regarding:

Figure 3 from "Estimating the minimum stimulation frequency necessary to evoke tetanic progression based on muscle twitch parameters"

We are happy to grant permission for the use you request on the terms set out below.

\section{License to publish material published by IOP Publishing}

\section{Conditions}

Non-exclusive, non-transferrable, revocable, worldwide, permission to use the material in print and electronic form will be granted subject to the following conditions:

- Permission will be cancelled without notice if you fail to fulfil any of the conditions of this letter.

- You will make reasonable efforts to contact the author(s) to seek consent for your intended use. Contacting one author acting expressly as authorised agent for their co-authors is acceptable.

- You will reproduce the following prominently alongside the material:

- the source of the material, including author, article title, title of journal, volume number, issue number (if relevant), page range (or first page if this is the only information available) and date of first publication. This information can be contained in a footnote or reference note; or

- a link back to the article (via DOI); and

- if practical and IN ALL CASES for works published under any of the Creative Commons licences the words "O(O) Institute of Physics and Engineering in Medicine. Reproduced by permission of IOP Publishing. All rights reserved"

- The material will not, without the express permission of the author(s), be used in any way which, in the opinion of IOP Publishing, could distort or alter the author(s)' original intention(s) and meaning, be prejudicial to the honour or reputation of the author(s) and/or imply endorsement by the author(s) and/or IOP Publishing and/or Institution of Physics and Engineering in Medicine.

- Payment of $€ 0$ is received in full by IOP Publishing prior to use.

This permission does not apply to any material/figure which is credited to another source in our publication or has been obtained from a third party. Express permission for such materials/figures must be obtained from the copyright owner.

Kind regards,

Sophie

Copyright \& Permissions Team

Figure 8: Permission granted from IOP Publishing to use the figures from "Estimate the minimum stimulation frequency necessary to evoke tetanic progression based on muscle twitch parameters" by S. Watanabe, et al. 



\section{References}

[1] P. S. Dias, J. S. Fort, D. A. Marinho, A. Santos, and M. C. Marques, "Tensiomyography in physical rehabilitation of high level athletes," The Open Sports Sciences Journal, vol. 3, no. 1, pp. 47-48, 2010.

[2] J. M. García-Manso, D. Rodríguez-Ruiz, D. Rodríguez-Matoso, S. de Yves, S. Sarmiento, and M. Quiroga, "Assessment of muscle fatigue after an ultraendurance triathlon using tensiomyography (TMG)," Journal of Sports Sciences, vol. 29, no. 6, pp. 619-625, 2011.

[3] E. Rey, C. Lago-Peñas, and J. Lago-Ballesteros, "Tensiomyography of selected lower-limb muscles in professional soccer players," Journal of Electromyography and Kinesiology, vol. 22, no. 6, pp. 866-872, 2012.

[4] R. Dahmane, S. Djordjevic, B. Simunic and V. Valencic, "Spatial fiber type distribution in normal human muscle Histochemical and tensiomyographical evaluation," Journal of Biomech., vol. 38, no. 12, pp. 2451-2459, 2005.

[5] N. Z. Mohamad, N. A. Hamzaid, G. M. Davis, A. K. Abdul Wahab, and N. Hasnan, "Mechanomyography and torque during FES-evoked muscle contractions to fatigue in individuals with spinal cord injury," Sensors (Switzerland), vol. 17, no. 7, pp. 1-16, 2017. 
[6] O. G. Garcia, "The relationship between parameters of tensiomyography and potential performance indicators in professional cyclists," Rev Int Med Cienc Act Fl's Deporte., vol. 13, no. 52, pp. 771-781, 2013.

[7] S. Gil, I. Loturco, V. Tricoli, C. Ugrinowitsch, R. Kobal and C. C. Abad Cal, "Tensiomyography parameters and jumping and sprinting performance in Brazilian elite soccer players. ," Sports Biomech., vol. 14, no. 3, pp. 340-350, 2015.

[8] L. J. Macgregor, A. M. Hunter, C. Orizio, M. M. Fairweather, and M. Ditroilo, "Assessment of skeletal muscle contractile properties by radial displacement: The case for tensiomyography," Sports Medicine, vol. 48, no. 7, pp. 1607-1620, 2018.

[9] W. Xi and X. Tao, "Continuous monitoring and modeling contractility of skeletal muscles in motion," Medical Research Archives, vol. 6, no. 6, 2018.

[10] J. Silver, Y. Ono, and A. Adler, "An ultrasonic technique for imaging of tissue motion due to muscle contraction," IEEE Ultrasonics Symposium, pp. 2441$2444,2009$.

[11] T. Koo, C. Wong and Y. Zheng, "Reliability of sonomyography for pectoralis major thickness measurement," J Manipulative Physiol Ther., vol. 33, pp. 386$394,2010$.

[12] A. Wong, K. M. Gallagher and J. P. Callaghan, "Computerised system for measurement of muscle thickness based on ultrasonography," Comput. Methods Biomech. Biomed. Engin., vol. 16, pp. 249-255, 2013. 
[13] A. Huang and Y. Ono, "Estimation of wrist flexion angle from muscle thickness changes measured by a flexible ultrasonic sensor," 2016 IEEE-EMBS International Conference on Biomedical and Health Informatics (BHI), pp. 188-191, 2016 .

[14] I. AlMohimeed, H. Turkistani, and Y. Ono, "Development of wearable and flexible ultrasonic sensor for skeletal muscle monitoring," pp. 1137-1140, 2013.

[15] I. AlMohimeed and Y. Ono, "Flexible and wearable ultrasonic sensor for assessment of skeletal muscle contractile properties," 2019 IEEE International Conference on Flexible and Printable Sensors and Systems, pp. 1-3, 2019.

[16] D. Rassier, R. Macintosh and W. Herzog, "Length dependence of active force production in skeletal muscle," American Physiological Society, pp. 1445-1457, 1999.

[17] H. Turkistani, I. Almohimeed and Y.Ono, "Ultrasonic signal processing for continuous measurements of tissue displacement and thickness during muscle contraction ," CMBEC, no. 36, pp. 21-24, 2013.

[18] I. Y. Kuo and B. E. Ehrlich, "Signaling in muscle contraction," Cold Spring Harbor Perspectives in Biology, vol. 7, no. 2, pp. 1-14, 2015.

[19] Q. Yang, X. Wang, Y. Fang, V. Siemionow, W. Yao and G. Yue, "Timedependent cortical activation in voluntary muscle contraction," The Open Neuroimaging Journal, vol. 5, pp. 232-239, 2011.

[20] J. G. Sennler, "Motor unit synchronization and neuromuscular performance," Exercise and sport sciences reviews, vol. 30, no. 1, pp. 8-14, 2002. 
[21] T.Mano and T. Matsukawa, "Muscular sympathetic nervous activity and automatic nervous system diseases," Nihon Naika Gakkai zasshi. The Journal of the Japanese Society of Internal Medicine, vol. 84, no. 4, pp. 645-650, 1995.

[22] W. Craig and R. Sebastien, "Human muscle fatigue,", Routledge, pp. 63-102, 2009.

[23] K. Yoshida, Y. Itoigawa, Y. Maruyama and K. Kaneko, "Healing process of gastrocnemius muscle injury on ultrasonography Using B-Mode imaging, power doppler imaging, and shear wave elastography," Journal of Ultrasound in Medicine, vol. 38, no. 12, pp. 3239-3246, 2019.

[24] T. Deffieux, J. Gennisson, M. Tanter, and M. Fink, "Assessment of the mechanical properties of the musculoskeletal system using 2-d and 3-d very high frame rate ultrasound," IEEE Transactions on Ultrasonics, Ferroelectrics, and Frequency Control, vol. 55, pp. 2177-2190, October 2008.

[25] I. Loturco, L. A. Pereira, R. Kobal, K. Kitamura, R. Ramírez-Campillo, V. Zanetti, C. C. Cal Abad, and F. Y. Nakamura, "Muscle contraction velocity: A suitable approach to analyze the functional adaptations in elite soccer players," Journal of Sports Science and Medicine, vol. 15, no. 3, pp. 483-491, 2016.

[26] O. G. Garcia, A. C. Dorado, T. A. Yates, J. C. Lopez and M. I. Caamano, "The relationship between parameters of tensiomyography and potential performance indicators in professional cyclists," Open Access Journal of Sports Medicine, vol. 10, pp. 49-69, 2019.

[27] S. Gehlert, S. Weber, B. Weidmann, K. Gutsche, P. Platen, C. Graf, K. K. Horn, W. Bloch and S. Gehlert, "The relationship between parameters of ten- 
siomyography and potential performance indicators in professional cyclists," European journal of applied physiology, vol. 112, no. 7, pp. 393-2402, 2012.

[28] L. M. Biga, S. Dawson, A. Harwell, R. Hopkins, J. Kaufmann, M. LeMaster, P. Matern, K. Morrison-Graham, D. Quick, and J. Runyeon, "Anatomy \& physiology: Chapter 10.2 skeletal muscle." OpenStax/Oregon State University [Online]. Available from: https ://open.oregonstate.education/aandp/ chapter/10-2-skeletal-muscle/. [Accessed: 23-Mar-2020].

[29] S. M. Walker and G. R. Schrodt, "I segment lengths and thin filament periods in skeletal muscle fibers of the rhesus monkey and the human," The Anatomical Record, vol. 178, no. 1, pp. 63-81, 1974.

[30] G. Copper, "Microtubule motors and movements," in The Cell: A Molecular Approach. 2nd edition, Oct 2000.

[31] P. F. Dillon, M. O. Aksoy, S. P. Driska, and R. A. Murphy, "Myosin phosphorylation and the cross-bridge cycle in arterial smooth muscle," vol. 211, no. 4481, pp. 495-497, 1981.

[32] G. Karki, "Sliding filament theory of muscle contraction." Online Biology Notes [Online]. Available from: https://www.onlinebiologynotes.com/ sliding-filament-model-of-muscle-contraction/. [Accessed: 23-Mar2020].

[33] V. S. Deshpande, R. M. McMeeking, and A. G. Evans, "A bio-chemo-mechanical model for cell contractility," Proceedings of the National Academy of Sciences of the United States of America, vol. 103, no. 38, pp. 14015-14020, 2006. 
[34] S. M. Baylor and S. Hollingworth, "Calcium indicators and calcium signalling in skeletal muscle fibres during excitation-contraction coupling," Progress in Biophysics and Molecular Biology, vol. 105, no. 3, pp. 162-179, 2011.

[35] R. Qaisar, S. Bhaskaran, and H. Van Remmen, "Muscle fiber type diversification during exercise and regeneration," Free Radical Biology and Medicine, vol. 98, pp. 56-67, 2016.

[36] O. College, "Anatomy and Physiology: Skeletal Muscle." OpenStax textbook [Online]. Available from: https://commons.wikimedia.org/wiki/File: 1012_Muscle_Twitch_Myogram.jpg, 2016. [Accessed: 23-Mar-2020].

[37] R. Mannan, "Genesis of tetanus, lecture slide 1." Department of physiology JNMC, AMU [Online]. Available from: https://www.slideshare.net/ raihan6112/genesis-of-tetanus, 2018. [Accessed: 23-Mar-2020].

[38] R. Raikova, M. Pogrzebna, H. Drzymała, J. Celichowski, and H. Aladjov, "Variability of successive contractions subtracted from unfused tetanus of fast and slow motor units," Journal of Electromyography and Kinesiology, vol. 18, no. 5, pp. $741-751,2008$.

[39] K. G. J. Celichowski and E. Bichler, "Changes in fusion index during the fatigue test of fast motor units in the medial gastrocnemius muscle of the rat," Nencki Inst. Experimental Biology, vol. 56, no. 4, pp. 881 -887, 1996.

[40] R. M. Enoka and D. G. Stuart, "Neurobiology of muscle fatigue," Journal of Applied Physiology, vol. 72, no. 5, pp. 1631-1648, 1992. 
[41] X. B. Li, J. D. Gu, and Q. H. Zhou, "Review of aerobic glycolysis and its key enzymes - new targets for lung cancer therapy," Thoracic Cancer, vol. 6, no. 1, pp. 17-24, 2015.

[42] D. G. Allen, G. D. Lamb, and H. Westerblad, "Skeletal muscle fatigue: Cellular mechanisms," Physiological Reviews, vol. 88, no. 1, pp. 287-332, 2008.

[43] S. Cooper and J. Eccles, "The isometric responses of mammalian muscles," The Journal of Physiology, vol. 4, no. 69(4), pp. 377-385, 1930.

[44] S. Watanabe, S. Fukuhara, T. Fujinaga, and H. Oka, "Estimating the minimum stimulation frequency necessary to evoke tetanic progression based on muscle twitch parameters," Physiological Measurement, vol. 38, no. 3, pp. 466-476, 2017.

[45] R. Qaisar, S. Bhaskaran, and H. Van Remmen, "Muscle fiber type diversification during exercise and regeneration," Free Radical Biology and Medicine, vol. 98, pp. 56-67, 2016.

[46] P. Siao, D. Cros and S. Vucic, ""Practical approach to electromyography an illustrated guide for clinicians"," New York : Demos Medical, 2010.

[47] J. M. Wakeling, M. Kaya, G. K. Temple, I. A. Johnston, and W. Herzog, "Determining patterns of motor recruitment during locomotion," Journal of Experimental Biology, vol. 205, no. 3, pp. 359-369, 2002.

[48] T. Yoneda, K. Oishi, S. Fujikura, and A. Ishida, "Recruitment threshold force and its changing type of motor units during voluntary contraction at various speeds in man," Brain Research, vol. 372, no. 1, pp. 89-94, 1986. 
[49] E. J. Kupa, S. H. Roy, S. C. Kandarian, and C. J. De Luca, "Effects of muscle fiber type and size on EMG median frequency and conduction velocity," Journal of Applied Physiology, vol. 79, no. 1, pp. 23-32, 1995.

[50] B. C. Clark, T. M. Manini, D. J. Thé, N. A. Doldo, and L. L. Ploutz-Snyder, "Gender differences in skeletal muscle fatigability are related to contraction type and EMG spectral compression," Journal of Applied Physiology, vol. 94, no. 6, pp. 2263-2272, 2003.

[51] G. Rau and C. D. Klug, "Principles of high-spatial-resolution surface EMG (HSR-EMG): single motor unit detection and application in the diagnosis of neuromuscular disorders.," J Electromyogr Kinesiol., vol. 7, no. 4, pp. 233-239, 1997.

[52] E. Cè, S. Rampichini, and F. Esposito, "Novel insights into skeletal muscle function by mechanomyography: from the laboratory to the field," Sport Sciences for Health, vol. 11, no. 1, pp. 1-28, 2015.

[53] M. O. Ibitoye, N. A. Hamzaid, J. M. Zuniga, N. Hasnan, and A. K. A. Wahab, "Mechanomyographic parameter extraction methods: An appraisal for clinical applications," Sensors (Switzerland), vol. 14, no. 12, pp. 22940-22970, 2014.

[54] T. Uchiyama and K. Shinohara, "Comparison of displacement and acceleration transducers for the characterization of mechanics of muscle and subcutaneous tissues by system identification of a mechanomyogram," Medical and Biological Engineering and Computing, vol. 51, no. 1-2, pp. 165-173, 2013.

[55] C. Than, L. Seidl, D. Tosovic, and J. M. Brown, "Test-retest reliability of laser displacement mechanomyography in paraspinal muscles while in lumbar 
extension or flexion," Journal of Electromyography and Kinesiology, vol. 41, no. October 2017, pp. 60-65, 2018.

[56] C. Than, L. Roberts, B. K. Coombes, D. Tosovic, and J. M. Brown, "Exerciseinduced muscle damage on the contractile properties of the lumbar paraspinal muscles: a laser displacement mechanomyographic approach," European Journal of Applied Physiology, vol. 119, no. 3, pp. 761-770, 2019.

[57] J. D. Manera, J. Llauger, E. Gallardo, and I. Illa, "Muscle MRI in muscular dystrophies," Acta Myologica, vol. 34, pp. 95-108, 2014.

[58] Y. Nagano, A. Higashihara, and M. Edama, "Change in muscle thickness under contracting conditions following return to sports after a hamstring muscle strain injury - A pilot study," Asia-Pacific Journal of Sports Medicine, Arthroscopy, Rehabilitation and Technology, vol. 2, no. 2, pp. 63-67, 2015.

[59] E. Silvesttri, A. Muda and D. Orlandi, "Ultrasound Anatomy of Lower Limb Muscles A Practical Guide," Springer International, 2015.

[60] T. Abe, M. Nakatani and J. P. Loenneke, "Relationship between ultrasound muscle thickness and MRI-measured muscle cross-sectional area in the forearm: a pilot study," Clinical Physiology and Functional Imaging, vol. 38, no. 4, pp. $652-655,2018$.

[61] P. W. Hodges, L. H. M. Pengel, R. D. Herbert, and S. C. Gandevia, "Measurement of muscle contraction with ultrasound imaging," Muscle and Nerve, vol. 27, no. 6, pp. 682-692, 2003.

[62] J. M. Scott, D. S. Martin, R. Ploutz-Snyder, T. Matz, T. Caine, M. Downs, K. Hackney, R. Buxton, J. W. Ryder, and L. Ploutz-Snyder, "Panoramic ultra- 
sound: a novel and valid tool for monitoring change in muscle mass," Journal of Cachexia, Sarcopenia and Muscle, vol. 8, no. 3, pp. 475-481, 2017.

[63] J. Shi, Z. Yan, Y, Zheng, "Evaluation of the muscle fatigue based on ultrasound images," in Proceedings of the World Congress on Intelligent Control and Automation (WCICA), vol. 2, pp. 10332 - 10336, 2006.

[64] J. R. Bagley, K. A. McLeland, J. A. Arevalo, L. E. Brown, J. W. Coburn, A. J. Galpin, "Skeletal Muscle Fatigability and Myosin Heavy Chain Fiber Type in Resistance Trained Men," Journal of Strength and Conditioning Research, vol. 31, no. 3, pp. $602-607,2017$.

[65] A. B. Wolbarst, "Physics of Radiology," Appleton and Lange, pp. 84-85, 1993.

[66] D. R. Dance, S. Christofides, A. D. A. Maidment, I. D. Mclean and K. H. Ng, Digital Image Processing for Medical Applications. International Atomic ENergy Agency Vienna, 2014.

[67] G. Dougherty, Digital Image Processing for Medical Applications. Cambridge University Press, 2009.

[68] G. H. Pascal Laugier, "Ultrasound, Bone Quantitative," Springer Dordrecht Heidelberg London New York, pp. 29-46, 2011.

[69] D. W. Rickey, "Medical ultrasound linear array probe/scan head/transducer." Wikimedia commons [Online]. Available from:https://commons.wikimedia. org/wiki/File:UltrasoundProbe2006a.jpg, 2006. [Accessed: 23-Mar-2020].

[70] I. AlMohimeed, H. Turkistani, and Y. Ono, "Development of wearable and flexible ultrasonic sensor for skeletal muscle monitoring," in 2013 IEEE International Ultrasonics Symposium (IUS), pp. 1137-1140, July 2013. 
[71] I. AlMohimeed, M. Agarwal, and Y. Ono, "Wearable ultrasonic sensor using double-layer pvdf films for monitoring tissue motion," in 2018 IEEE Canadian Conference on Electrical Computer Engineering (CCECE), pp. 1-4, May 2018.

[72] I. Measurement Specialties, Piezo Film Sensors Technical Manual. Measurement Specialties, Inc, Canada.

[73] P. E. Bloomfield, "Experimental study of the acoustical properties of polymers utilized to construct PVDF ultrasonic transducers and the acousto-electric properties of PVDF and $\mathrm{P}(\mathrm{VDF} / \mathrm{TrFE})$ films," IEEE Transactions on Ultrasonics, Ferroelectrics, and Frequency Control, vol. 47, no. 6, pp. 1397-1405, 2000.

[74] W. Lee and Y. Roh, "Ultrasonic transducers for medical diagnostic imaging," Biomedical Engineering Letters, vol. 7, no. 2, pp. 91-97, 2017.

[75] D. Hutchins and A. Neild, "Ultrasonic Transducers," Woodhead Publishing, pp. 374-407, 2012.

[76] M. Chemicals, 8331 SILVER CONDUCTIVE EPOXY ADHESIVE KIT. MG Chemicals, Burlington, Ontario, Canada.

[77] K. M. P. Hoskins and A. Thrush, "Diagnostic Ultrasound Physics and Equipment (second Edition)," Cambridge University Press, pp. 15-17, 2010.

[78] S. B. Murthi, M. Ferguson, and A. C. Sisley, Ultrasound physics and equipment. 2010.

[79] J. Krautkraemer, H. Krautkramer and W. Grabendoerfer, "Ultrasonic testing of materials.," Berlin: Springer Verlag., pp. 71-73, 1989. 
[80] G. E. Doxey, "The association of anthropometric measurements of thigh size and B-mode ultrasound scanning of muscle thickness," Journal of Orthopaedic and Sports Physical Therapy, vol. 8, no. 9, pp. 462-468, 1987.

[81] Y. Kawakami, T. Abe, H. Kanehisa, and T. Fukunaga, "Short Report Human Skeletal Muscle Size and Architecture: Variability and Interdependence.," American Journal of Human Biology, vol. 18, no. 18, pp. 845-848, 2006.

[82] F. Zabihollahy, B. M. Trindade, Y. Ono, and E. D. Lemaire, "Continuous monitoring of mechanical properties of plantar soft tissue for diabetic patients using wearable ultrasonic and force sensors," 2016 IEEE EMBS International Student Conference: Expanding the Boundaries of Biomedical Engineering and Healthcare, ISC 2016 - Proceedings, 2016.

[83] H. Nyquist, "Certain topics in telegraph transmission theory," Trans. AIEE., vol. 47, no. 2, pp. 617-644, 1928.

[84] N. Feng, J. Zhang, and W. Wang, "A quadrature demodulation method based on tracking the ultrasound echo frequency," Ultrasonics, vol. 44, no. SUPPL., pp. $47-50,2006$.

[85] H. Hao and Y. F. Zheng, "An efficient quadrature demodulator for medical ultrasound imaging.," Frontiers Inf Technol Electronic Eng, vol. 16, no. 16, pp. 301-310, 2015.

[86] Y. Deng, N. C. Rouze, M. L. Palmeri and K. R. Nightingale, "Ultrasonic Shear Wave Elasticity Imaging (SWEI) Sequencing and Data Processing Using a Verasonics Research Scanner.," IEEE Transactions on ultrasonics, ferroelectrics, and frequency control, vol. 1, no. 64, pp. 164-176, 2017. 
[87] K. Nightingale, "Acroustic Radiatiom Force Impulse (ARFI) imaging: a Review.," Current medical imaging reviews, vol. 4, no. 7, pp. 328-339, 2011.

[88] J. Y. Guo, Y. P. Zheng, Q. H. Huang, and X. Chen, "Dynamic monitoring of forearm muscles using one-dimensional sonomyography system," Journal of Rehabilitation Research and Development, vol. 45, no. 1, pp. 187-196, 2008.

[89] J. J. Ammann and B. Galaz, "Sound velocity determination in gel-based emulsions," Ultrasonics, vol. 41, no. 7, pp. 569-579, 2003.

[90] M. O. Culjat, D. Goldenberg, P. Tewari, and R. S. Singh, "A review of tissue substitutes for ultrasound imaging," Ultrasound in Medicine and Biology, vol. 36, no. 6, pp. 861-873, 2010.

[91] J. R. Cook, R. R. Bouchard, and S. Y. Emelianov, "Tissue-mimicking phantoms for photoacoustic and ultrasonic imaging," Biomedical Optics Express, vol. 2, no. 11, p. 3193, 2011.

[92] C. Richardson, S. Bernard, and V. A. Dinh, "A cost-effective, gelatin-based phantom model for learning ultrasound-guided fine-needle aspiration procedures of the head and neck," Journal of Ultrasound in Medicine, vol. 34, no. 8, pp. 1479-1484, 2015.

[93] Y. Zhu, C. Dong, Y. Yin, X. Chen, Y. Guo, Y. Zheng, Y. Shen, T. Wang, X. Zhang and S. Chen, "Computerised system for measurement of muscle thickness based on ultrasonography," Ultrasound in Medicine and Biology, vol. 41, no. 2, pp. 601-609, 2015.

[94] J. F. Gerstenmaier, C. J. Mccarthy, D. P. Brophy and C. P. Cantwell, "Evaluation of the Particulate Concentration in a Gelatin-Based Phantom for Sono- 
graphically Guided Lesion Biopsy," Journal of Ultrasound in Medicine, vol. 32, no. 8, pp. $1471-1475,2013$.

[95] I. C. on Radiation Units and Measurements, "Tissue substitutes,phantoms,and computational modelling in medical ultrasound," Bethesda, pp. 60-65, 1998.

[96] A. Sakai, Y. Murayama, K. Fujiwara, T. Fujisawa, S. Sasaki, S. Kidoaki, and M. Yanagisawa, "Increasing Elasticity through Changes in the Secondary Structure of Gelatin by Gelation in a Microsized Lipid Space," ACS Central Science, vol. 4, no. 4, pp. 477-483, 2018.

[97] Y. N. Feng, Y. P. Li, C. L. Liu, and Z. J. Zhang, "Assessing the elastic properties of skeletal muscle and tendon using shearwave ultrasound elastography and MyotonPRO," Scientific Reports, vol. 8, no. 1, pp. 1-9, 2018.

[98] S. A. Lopez-Haro, I. Bazan-Trujillo, L. Leija-Salas, and A. Vera-Hernandez, "Ultrasound propagation speed measurement of mimicking soft tissue phantoms based on agarose in the range of $25^{\circ} \mathrm{c}$ to $50^{\circ} \mathrm{c}, "$ in 2008 th International Conference on Electrical Engineering, Computing Science and Automatic Control, pp. 192-195, Nov 2008.

[99] N. P. Cheremisinoff, Condensed encyclopedia of polymer engineering terms. Butterworth-Heinemann, 2001.

[100] G. Destgeer, J. H. Jung, J. Park, H. Ahmed, K. Park, R. Ahmad and H. J. Sung, "Acoustic impedance-based manipulation of elastic microspheres using travelling surface acoustic waves," Royal Society of Chemistry, vol. 7, no. 7, pp. 22524-22530, 2017. 
[101] M. C. Overmoyer, B.A and G. Brittenham, "Uniformity of liver density and nonheme (storage) iron distribution.," Archives of Pathology and Laboratory Medicine, pp. 549 - 554, 1987.

[102] Keyence Cooperation, "Ultra high-speed/high-accuracy laser displacement sensor LK-G 5000 Series," Keyence, 2018.

[103] A. R. Kuntz, C. M. Griffiths, J. M. Rankin, C. W. Armstrong, and T. J. Mcloughlin, "Cortisol concentrations in human skeletal $10 \%$ hydrocortisone gel," vol. 41, no. 3, pp. 321-324, 2006.

[104] L. Poltawski and T. Watson, "Relative transmissivity of ultrasound coupling agents commonly used by therapists in the UK," Ultrasound in Medicine and Biology, vol. 33, no. 1, pp. 120-128, 2007.

[105] J. W. Myrer, G. J. Measom, and G. W. Fellingham, "Intramuscular Temperature Rises with Topical Analgesics Used as Coupling Agents during Therapeutic Ultrasound," Journal of Athletic Training, vol. 36, no. 1, pp. 20-26, 2001.

[106] N. G. Parker and M. J. Povey, "Ultrasonic study of the gelation of gelatin: Phase diagram, hysteresis and kinetics," Food Hydrocolloids, vol. 26, no. 1, pp. 99-107, 2012.

[107] E. Yeung, I. Almohimeed, and Y. Ono, "Estimation of tissue thickness changes due to electrical muscle stimulation using wearable ultrasonic sensor in pulse echo mode," IEEE, pp. 9-12, 2019.

[108] C. E. Efstathiou, "Signal smoothing algorithms." [Online]. Available from:http://195.134.76.37/applets/AppletSmooth/Appl_Smooth2.html. [Accessed: 23-Mar-2020]. 
[109] B. M. Doucet, A. Lam, and L. Griffin, "Neuromuscular Electrical Stimulation for Skeletal Muscle Function," Yale Journal of Biology and Medicine, vol. 85, no. 2, pp. 201-215, 2012.

[110] D. Weiss, S. Breit, J. Hoppe, A.-K. Hauser, D. Freudenstein, R. Krüger, P. Sauseng, R. B. Govindan, and C. Gerloff, "Subthalamic nucleus stimulation restores the efferent cortical drive to muscle in parallel to functional motor improvement," European Journal of Neuroscience, vol. 35, no. 6, pp. 896-908, 2012.

[111] J. Gomdin , M. Guette, Y. Balay, et al, "Electromyostimulation training effects on neural drive and muscle architecture.," Med Sci Sports Exercise, vol. 37, no. 1, pp. 1291-1299, 2005.

[112] N. A. Maffiuletti, S. Dugnani, M. Folz, et al., "Effect of combined eletrostimulation and plyometric training on vertical jump height.," Med Sci Sports Exercise, vol. 34, no. 1, pp. 1638-1644, 2002.

[113] F. Brocherie, N. Babault, G. Cometti, N. Maffiuletti, and J. C. Chatard, "Electrostimulation training effects on the physical performance of ice hockey players," Medicine and Science in Sports and Exercise, vol. 37, no. 3, pp. 455-460, 2005.

[114] C. J. Mitchell and D. G. Sale, "Enhancement of jump performance after a 5RM squat is associated with postactivation potentiation," European Journal of Applied Physiology, vol. 111, no. 8, pp. 1957-1963, 2011.

[115] T. Wiewelhove, C. Raeder, T. Meyer, M. Kellmann, M. Pfeiffer, and A. Ferrauti, "Markers for routine assessment of fatigue and recovery in male and female 
team sport athletes during high-intensity interval training," PLoS ONE, vol. 10, no. 10, pp. 1-18, 2015.

[116] E. Alentorn-Geli, R. Seijas, A. Martínez-De la Torre, X. Cuscó, G. Steinbacher, P. Álvarez-Díaz, D. Barastegui, J. Navarro, J. M. Serra-Renom, B. Nishishinya, J. Català, P. Laiz, M. García-Balletbó, and R. Cugat, "Effects of autologous adipose-derived regenerative stem cells administered at the time of anterior cruciate ligament reconstruction on knee function and graft healing," Journal of Orthopaedic Surgery, vol. 27, no. 3, pp. 1-8, 2019.

[117] K. Avramidis, P. W. Strike, P. N. Taylor, and I. D. Swain, "Effectiveness of electric stimulation of the vastus medialis muscle in the rehabilitation of patients after total knee arthroplasty," Archives of Physical Medicine and Rehabilitation, vol. 84, no. 12, pp. 1850-1853, 2003.

[118] T. Paillard, "Combined application of neuromuscular electrical stimulation and voluntary muscular contractions," Sports Medicine, vol. 38, no. 2, pp. 161-177, 2008.

[119] S. Tanaka, K. Kamiya, Y. Matsue, R. Yonezawa, H. Saito, N. Hamazaki, R. Matsuzawa, K. Nozaki, K. Wakaume, Y. Endo, E. Maekawa, M. YamaokaTojo, T. Shiono, T. Inomata, T. Masuda, and J. Ako, "Effects of acute phase intensive electrical muscle stimulation in frail elderly patients with acute heart failure (ACTIVE-EMS): rationale and protocol for a multicenter randomized controlled trial," Clinical Cardiology, vol. 40, no. 12, pp. 1189-1196, 2017.

[120] Y. Laufer, J. D. Ries, P. M. Leininger and G. Alon, "Quadriceps femoris muscle torques and fatigue generated by neuromuscular electrical stimulation with 
three different waveforms," Physical Therapy, vol. 81, no. 7, pp. 1307-1316, 2001.

[121] W. M. Grill and J. T. Mortimer, "The effect of stimulus pulse duration on selectivity of neural stimulation," IEEE Transactions on Biomedical Engineering, vol. 43, no. 2, pp. 161-166, 1996.

[122] V. Valenčič and N. Knez, "Measuring of skeletal muscles' dynamic properties ,"Artificial Organs, vol. 21, no. 3, pp. 240-242, 1997.

[123] R. Seijas, M. Marín, E. Rivera, E. Alentorn-Geli, D. Barastegui, P. ÁlvarezDíaz, and R. Cugat, "Gluteus maximus contraction velocity assessed by tensiomyography improves following arthroscopic treatment of femoroacetabular impingement," Knee Surgery, Sports Traumatology, Arthroscopy, vol. 26, no. 3, pp. 976-982, 2018.

[124] S. Jacob, "Human Anayomy - Upper Arm," Diagnostic Ultrasound Physics and Equipments, pp. 5-49, 2008.

[125] W. Platzer and G. Spitzer, "Color Atlas of Human Anatomy: Locomotor system," Thieme, 2009.

[126] Anatomography, "File:Triceps brachii muscle06.png." Wikimedia [Online]. Available from: https://commons.wikimedia.org/wiki/File:Triceps_ brachii_muscle06.png, 2013. [Accessed: 23-Mar-2020].

[127] S. K. Carol Najarian and R. A. Peura, "Effects of Current Frequency Fiber Orientation, and Sample Thickness on the Measured resistivity of Skeletal Muscle," Proceedings of 1994 20th Annual Northeast Bioengineering Conference. IEEE, vol. 0, no. 1, pp. 118-119, 1994. 
[128] J. E. Conklin, "Three Factors Affecting the General Level of Electrical SkinResistance.," The American journal of psychology, vol. 54, no. 1, pp. 78-86, 1951.

[129] H. P. Schewan and C. F. Kay, "Specific Resistance of Body Tissues," Circulation Research, vol. 4, no. 1, pp. 664-670, 1956.

[130] M. G. Claudio Orizio and B. Diemont, "Changes of the force-frequency relationship in human tibialis anterior at fatigue," Journal of Electromyography and Kinesiology, vol. 14, no. 5, pp. 523-530, 2004.

[131] W. Mrowczynski, J. Celichowski and P. Krutki, "Interspecies differences in the force-frequency relationship of the medial gastrocnemius motor units," J Physiol Pharmacol, vol. 57, no. 3, pp. 491-501, 2006.

[132] S. Watanabe, T. Kitawaki and H. Oka, "The relationship between fusion indexfrequency curve of displacement-MMG and fiber composition of skeletal muscle," Baiomekanizumu., vol. 20, no. 2, pp. 207-216, 2010.

[133] J. J. Widrick, S. W. Trappe, C. A. Blaser, D.L.Costill and R.H.Fitts, "Isometric force and maximal shortening velocity of single muscle fibers from elite master runners," Am. J. Physiol., vol. 271, no. 1, pp. 264-274, 1996.

[134] Z. Yan, M. Okutsu, Y. N. Akhtar, and V. A. Lira, "Regulation of exerciseinduced fiber type transformation, mitochondrial biogenesis, and angiogenesis in skeletal muscle," Journal of Applied Physiology, vol. 110, no. 1, pp. 264-274, 2010.

[135] M. J. Toth, M. S. Miller, P. VanBuren, N. G. Bedrin, M. M. LeWinter, P. A. Ades and B. M. Palmer, "Resistance training alters skeletal muscle structure 
and function in human heart failure: effects at the tissue, cellular and molecular levels," J. Physiol, vol. 590, no. 3, pp. 1371-1375, 2012.

[136] W. Qiu, Z. Ye, Y. Yu, Y. Chen, L. Chi, P. Mu, G. Li, C. Wang, Y. Xiao, J. Dai, L. Sun, and H. Zheng, "A Digital Multigate Doppler Method for High Frequency Ultrasound," Sensors, vol. 14, no. 1, pp. 13348-13360, 2014.

[137] P. Cooperation, Compact Laser Displacement Sensor HL-G1. Panasonic.

[138] S. A. O'Leary, B. J. Doyle, and T. M. McGloughlin, "Comparison of methods used to measure the thickness of soft tissues and their influence on the evaluation of tensile stress," Journal of Biomechanics, vol. 46, no. 11, pp. 1955-1960, 2013.

[139] S. Moghimi, S. Kiumehr, J. Amoozadeh, and G. Fakhraie, "Axial length measurement in asteroid hyalosis: Comparing laser interferometry and ultrasound A-scan," Iranian Journal of Ophthalmology, vol. 21, no. 1, pp. 29-34, 2009.

[140] N. Nitta, T. Aoki, K. Hyodo, M. Misawa, and K. Homma, "Direct measurement of speed of sound in cartilage in situ using ultrasound and magnetic resonance images," Proceedings of the Annual International Conference of the IEEE Engineering in Medicine and Biology Society, EMBS, pp. 6063-6066, 2013.

[141] J. Dong, Z. W. Liu, and J. X. Gao, "3D Deformation Measurement of Soft Material under Indentation Using Improved Diffraction-Assisted Image Correlation," Experimental Mechanics, vol. 58, no. 1, pp. 87-98, 2018.

[142] M. M. Narayanan, N. Singh, A. Kumar, C. Babu Rao, and T. Jayakumar, "An absolute method for determination of misalignment of an immersion ultrasonic transducer," Ultrasonics, vol. 54, no. 8, pp. 2081-2089, 2014. 